\title{
Sequential Norrish Type II Photoelimination and Intramolecular Aldol Cyclization of a-Diketones: Synthesis of Polyhydroxylated Cyclopentitols by Ring Contraction of Hexopyranose Carbohydrate Derivatives
}

\author{
Dimitri Alvarez-Dorta, ${ }^{[a]}$ Elisa I. León, ${ }^{*[a]}$ Alan R. Kennedy ${ }^{[b]}$ Angeles Martín, ${ }^{[a]}$ \\ Inés Pérez-Martín, ${ }^{[a]}$ Concepción Riesco-Fagundo, ${ }^{[a]}$ and Ernesto Suárez ${ }^{*[a]}$
}

\begin{abstract}
The excitation of the innermost carbonyl of nono-2,3-diulose derivatives by irradiation with visiblelight initiates a sequential Norrish type II photoelimination and aldol cyclization process that finally gives polyfunctionalized cyclopentitols. The rearrangement has been confirmed by the isolation of stable acyclic photoenol intermediates that can be independently cyclized by a thermal 5-(enolexo)-exotrig uncatalyzed aldol reaction with
\end{abstract}

high diastereoselectivity. In this last step, the large deuterium kinetic isotope effect found for the 1,5-hydrogen atom transfer seems to indicate that the aldol reaction runs through a concerted pericyclic mechanism. Owing to the ready availability of pyranose

Keywords: carbohydrates · cyclopentitols $\cdot$ diketones $\cdot$ photochemistry ring contraction sugars of various configurations, this protocol has been used to study the influence of pyranose ring-substituents on the diastereoselectivity of the aldol cyclization reaction. In contrast with other pyranose ring contraction methodologies no transition-metal reagents are needed and the sequential rearrangement occurs simply by using visible light and moderate heating ( 0 to 60 8C).

\section{Introduction}

The photochemical behavior of monoketones has attracted a great deal of attention from synthetic and theoretical perspectives, whereas comparatively much less evidence is available on $a$-diketones. ${ }^{[1]}$ Both types of photoexcited carbonyls exhibit a remarkable regioselectivity of intramolecular 1,5-hydrogen transfer, to give a 1,4-biradical intermediate, which finally evolves following two competitive paths: Norrish type II photoelimination and Norrish-Yang photocyclization. ${ }^{[2]}$ Whereas photoelimination is usually the priority process with monoketones, a-diketones yield almost exclusively 2-hydroxycyclobutanones by photocyclization. Moreover, in $a$-diketones the 1,5-hydrogen abstraction apparently occurs only from the external carbonyl, such that the formation of acyl cyclobutanols has never been ob-

a. Dr. D. Alvarez-Dorta, Dr. E. I. León, Dr. A. Martín, Dr. I. Pérez-Martín, Dr. C. Riesco-Fagundo, Prof. Dr. E. Suárez Instituto de Productos Naturales, y Agrobiología del C.S. I.C. Carretera de la Esperanza 3, 38203 La Laguna, Tenerife (Spain) Fax: (+ 34) 922-260-135

E-mail: eila@ipna.csic.es esuarez@ipna.csic.es

b. Dr. A. R. Kennedy

WestCHEM Department of Pure and Applied Chemistry University of Strathclyde 295 Cathedral Street, Glasgow G1 1 XL (UK)

Supporting information for this article is available on the $\mathrm{WWW}$ under http://dx.doi.org/10.1002/chem.201301230. served. The development of synthetic applications of this 1,5hydrogen atom transfer reaction originated by $a$-dike- tones has been scant. ${ }^{[3]}$ This is somewhat surprising since $a$ diketones can be selectively activated over other photochemically excitable groups, just by irradiation with visible light (violet-blue interval, ca. $450 \mathrm{~nm}$ ). We believe this may be due to the lack of direct and general methods for their synthesis, especially on sensitive substrates, and to the instability observed for some aliphatic $a$-diketones.

The conversion of carbohydrate derivatives into enantiopure polyfunctionalized cyclopentanes has been extensively documented. ${ }^{[4]}$ Two general methodologies are currently used: Ring closing of polyfunctionalized acyclic molecules that most often involves multistep reaction sequences and ring contraction of pyranoside carbohydrate derivatives, which normally require a single synthetic step. These ringcontraction methods represent a highly efficient biomimetic approach to the synthesis of cyclopentanols. ${ }^{[5]}$ Several procedures have been published, all of which start from conveniently modified carbohydrate derivatives and in general involve a reductive ring-opening followed by an intramolecular reductive coupling using samarium or zirconium reagents. Representative examples with indication of starting materials and reagents are: Hept-6-enopyranosides $\left(\left[\mathrm{Cp}_{2} \mathrm{ZrCl}_{2}\right] / \mathrm{BF}_{3} \cdot \mathrm{OEt}_{2}\right)^{[6]}$ or $\left(\mathrm{SmI}_{2} /\left[\mathrm{Pd}\left(\mathrm{PPh}_{3}\right)_{4}\right]\right),{ }^{[7]}$ oct-6-enopyranosiduronate $\left(\mathrm{SmI}_{2}\right),{ }^{[8]}$ hept-6-ynopyranosides $\left(\mathrm{SmI}_{2} /\right.$ $\left.\left[\mathrm{Pd}\left(\mathrm{PPh}_{3}\right)_{4}\right]\right),{ }^{[7]}$ 6-deoxy-6-iodo-hexopyranoside $(\mathrm{SmI})_{2}^{\left[{ }^{[9]}\right.}$ and glucodialdo-1,5-hexopyranoside $\left(\mathrm{SmI}_{2}\right) .{ }^{[10]}$

In connection with our ongoing research programs on the reactivity of $a$-diketones ${ }^{[11]}$ and intramolecular hydrogen 


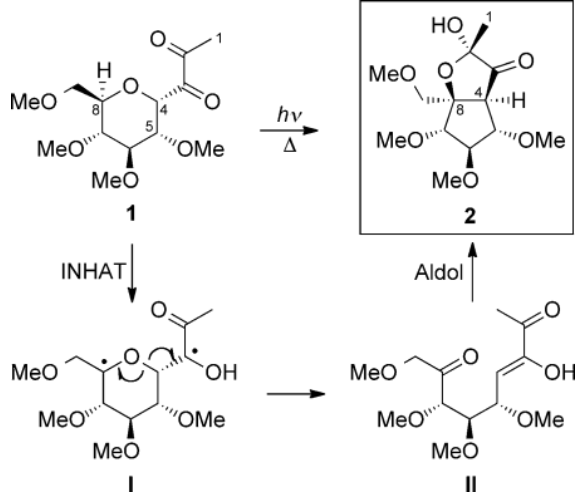

Scheme 1. Photochemistry of nono-2,3-diulose 1. IN HAT = intramolecular hydrogen atom transfer.

atom transfer (INHAT) promoted by alkoxyl radicals in carbohydrate chemistry, ${ }^{[12]}$ we present herein our results on the photochemical reactivity of nono-2,3-diuloses (Scheme 1). ${ }^{[13]}$ It is generally accepted that hydrogen atom abstraction by a photoexcited carbonyl closely resembles the hydrogen atom abstraction by alkoxyl radicals. ${ }^{[1, b]}$ Therefore, one would expect that in nono-2,3-diulose 1 , in as much as the hydrogen atom at $\mathrm{C} 5$ is geometrically inaccessible, the abstraction of H8 may proceed either by the external carbonyl (1,6HAT) or by the innermost carbonyl (1,5-HAT). Through research conducted in our laboratory we have shown that both types of processes can be carried out with alkoxyl radi- cals. $C$-Glycosides possessing 1-hydroxymethyl[14] and 1-hydroxyethy ${ }^{[15]}$ tethers cyclized, with the $\mathrm{PhI}(\mathrm{OAc})_{2} / \mathrm{I}_{2}$ system, to give 6,8-dioxabicyclo[3.2.1]octane (1,5-HAT) and 2,9dioxabicyclo[3.3.1]nonane (1,6-HAT) derivatives, respectively.

As described in Scheme 1 the reaction of 2,3-diulose 1 proceeded by a sequential Norrish type II photoelimination through the 3,8-biradical I; the intermediate photoenol II subsequently formed was converted into a cyclopentane derivative by a thermal $\mathrm{OH}-b$-syn aldol cyclization. Finally, the regenerated $a$-diketone reacts with the hydroxyl group at C8

to give the hemiketal 2 . Some features of these processes deserve brief comment. Only visible light and moderate heating are necessary for the reaction to take place, in contrast with the other ring contraction methods listed above that require the presence of transition-metal reagents. As mentioned before, Norrish type II photoelimination of $a$-diketones is an extremely rare reaction, whereas the uncatalyzed aldol cyclization, in which a photoenol acts as a preformed enolate, is unprecedented to our knowledge. As noted, the stereocenters at C4 and C8 destroyed in the photochemical reaction are regenerated diastereoselectively with inversion of configuration in the aldol cyclization step.

Owing to the ready availability of pyranose sugars of various configurations, this protocol would provide access to highly oxygenated 1,2-trans dialkylated cyclopentanes and cyclopentanoid natural products, for example, prostaglandins, brefeldin, and Corey lactone analogues. The synthesis of systems with 1-(hydroxymethyl)cyclopentitol moieties such as trehazoline, calditol, and bacteriohopanoids should be particularly accessible through this methodology. We believe that this protocol is also uniquely suited to study the effect of pyranose ring-substituents on the diastereoselectivity of the aldol cyclization reaction.

\section{Results and Discussion}

The present contribution is a study of the scope and the selectivity of this reaction, as well as an effort to establish the best conditions for this photoreaction and to demonstrate the mechanism involved. This has been achieved by prepar- ing a variety of 2,3-diuloses belonging to carbohydrates in pyranose and furanose form.

Synthesis of a- $C$-propynyl glycosides: We based the preparation of these compounds on the $a$-ethynylation of sugars by C-glycosidation using $n \mathrm{Bu}_{3} \mathrm{SnC} \div \mathrm{CSiMe}_{3}$ in the presence of trimethylsilyl triflate (TMSOTf) followed by desilylation of the initial adducts, a methodology well established by the groups of Isobe ${ }^{[16]}$ and Dondoni. ${ }^{[17]}$ Thus, gluco derivative 6 was obtained from acetate 3 through the silyl intermediate 5 in good yield (Scheme 2). Unfortunately, repeated attempts

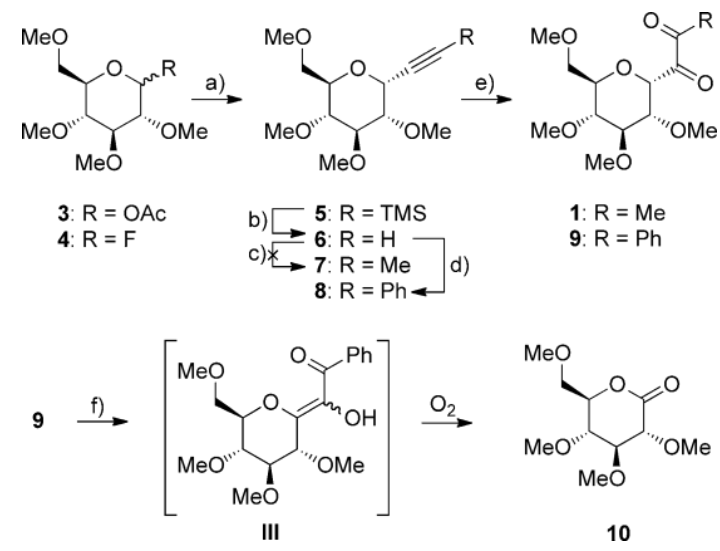

Scheme 2. Synthesis of nono-2,3-diuloses by oxidation of non-2-ynitols. a) For the synthesis of 5 from $3: n \mathrm{Bu}_{3} \mathrm{SnC} \div \mathrm{CTMS}$ (2 equiv), TMSOTf (2.5 equiv), $\mathrm{CH}_{2} \mathrm{Cl}_{2}$, MS $4 \AA$, room temperature, $3 \mathrm{~h}, 66 \%$; for the synthesis of 7 from 3 (Method $\mathrm{A}$ ): TMSC $\div \mathrm{CMe}$ (2 equiv), TMSOTf (3 equiv), MeCN, MS $4 \AA, 08 \mathrm{C}, 2 \mathrm{~h}, 33 \%$; for the synthesis of 7 from 3 (Method B): $n-\mathrm{Bu}_{3} \mathrm{SnC} \div \mathrm{CMe}$ (2 equiv), TMSOTf (2 equiv), $\mathrm{CH}_{2} \mathrm{Cl}_{2}$, MS $4 \AA$, room temperature, $2 \mathrm{~h}, 82 \%$; for the synthesis of 7 from $4: n-\mathrm{Bu}_{3} \mathrm{SnC} \div \mathrm{CMe}$ (2.5 equiv), $\mathrm{BF}_{3} \cdot \mathrm{OEt}_{2}$ (2 equiv), $\mathrm{CH}_{2} \mathrm{Cl}_{2}$, MS $4 \AA$, room temperature, $1 \mathrm{~h}$, $51 \%$; b) $\mathrm{NaOH}$ (1.9 equiv), $\mathrm{CH}_{2} \mathrm{Cl}_{2} / \mathrm{MeOH}$, room temperature, $2 \mathrm{~h}, 92 \%$; c) see text; d) $\left[\mathrm{Pd}\left(\mathrm{PPh}_{3}\right)_{4}\right],(0.05$ equiv), $\mathrm{CuI}$ (0.1 equiv), $\mathrm{PhI}(1$ equiv), piperidine, $808 \mathrm{C}, 1 \mathrm{~h}, 88 \%$; e) $\mathrm{O}_{3}$ (excess), $\mathrm{CH}_{2} \mathrm{Cl}_{2},-788 \mathrm{C}, 2 \mathrm{~h}$, then $\mathrm{Me}_{2} \mathrm{~S}$ (2.1 equiv), 6h, dark; f) Daylight-lamp irradiation, $\mathrm{C}_{6} \mathrm{D}_{6}, 308 \mathrm{C}, \mathrm{N}_{2}, 9 \mathrm{~h}$, then air, $48 \%$, two steps. TMS $=$ trimethylsilyl, 0 Ac $=$ acyl.

to accomplish the alkyne methylation by using several bases ( $n \mathrm{BuLi}, \mathrm{LDA}$, and $\mathrm{NaH}$ ) and alkylating agents (MeI and MeOTf) under a range of conditions gave none of the desired product 7 and only starting material 6 was recovered in all cases. ${ }^{[18]} \mathrm{A}$ related attempt at C-glycosidation by treat- 
ment of acetate 3 and commercially available TM SC $\div \mathrm{CMe}$ was similarly unproductive. After extensive experimentation (see the Supporting Information) the $a$ - $C$-propynyl glucoside 7 was obtained with good diastereoselectivity but in an unacceptable maximum yield of $33 \%$. Therefore, an alternative route was sought using $n \mathrm{Bu}_{3} \mathrm{SnC} \div \mathrm{CMe}$ as glycosyl acceptor, which was prepared according to the procedure developed by Logue and Teng. ${ }^{[19]}$ The reaction catalyzed by TMSOTf proceeded very smoothly to give 7 in good yield and diastereoselectivity ( $90 \%$, diastereomeric ratio (d.r.) $a / b$, 10:1). Alternatively, by using glucopyranosyl fluoride 4 as glycosyl donor and $\mathrm{BF}_{3} \cdot \mathrm{OEt}_{2}$ as catalyst the diastereoselectivity was improved since almost only the $a$-isomer of the product was detected. All the $a$ - $C$-propynyl glycosides shown in Tables 1-4 were prepared by using one of these two methodologies or in some cases by simple functional group modification between them. In general, the fluoride method was preferred in substrates with acid-sensitive pro- tectors (e.g., 15, Table 1), whereas the formation of highly unstable fluorides, as in the furanoses 67-69 (Table 4), pre- vents its utilization.

For comparative purposes in the photochemical experiments, permethylated 2,6-anhydro-7,8-dideoxy-8-phenyl-dglycero-l-gulo-oct-7-ynitol (9) was prepared by Sonogashira cross-coupling of alkyne 6 with iodobenzene. ${ }^{[20]}$

Diketones 1, 9 (Scheme 2), 18-24 (Table 1), 38-43 (Table 2), 58-61 (Table 3), and 70-72 (Table 4) were pre- pared from the corresponding non-2-ynitols by oxidation of the triple bond with ozone (Method $\mathrm{A})^{[21]}$ or $\mathrm{NaIO}_{4} / \mathrm{RuO}_{2} \cdot x$ $\mathrm{H}_{2} \mathrm{O}$ (Method B). ${ }^{[22]} a$-Diketones using benzyl ether as protective groups were better prepared by oxidation of the triple bond with $\mathrm{NaIO}_{4} / \mathrm{RuO}_{2} \cdot x \mathrm{H}_{2} \mathrm{O}$ than by using the ozone methodology. The $a$-diketones obtained as yellow oils are in most cases stable for at least several months when

stored at $-258 \mathrm{C}$ under nitrogen in the dark and can be purified by rapid silica gel column chromatography, although a significant loss of material was generally observed. In all the $a$-diketones the conformation of the pyranose ring was carefully studied by analysis of its vicinal coupling constant values. A chair ${ }^{7} C_{4}$ conformation (equivalent to ${ }^{4} C_{1}$ in the aldose series), in which the $\mathrm{H} 8$ and the diketone tether are in a 1,3-diaxial relationship, appears to be essential for the intramolecular hydrogen atom transfer (INHAT) reaction to take place.

In our preliminary experiment, a $\left[\mathrm{D}_{6}\right]$ benzene solution of a-diketone 1 was irradiated with a daylight lamp (Philips master PL electronic, $23 \mathrm{~W} / 865$ ) at $30 \mathrm{8C}$ until the yellow color faded $(3 \mathrm{~h})$. Direct sunlight can also be used but the reaction is usually slower and times are not reproducible. A single diastereomer with a hexahydro- $2 H$-cyclopenta $[b]$ furan bicyclic structure 2 was formed in $52 \%$ yield, and no other isomers were detected by ${ }^{1} \mathrm{H} N \mathrm{NR}$ spectroscopy of the crude reaction mixture (Table 1). With the aim of establish- ing the excitation behavior of the dicarbonyl system, a solu-
Table 1 . Synthesis and photolysis of 2,3-diuloses with $a$-d-glucopyranose stereochemistry.

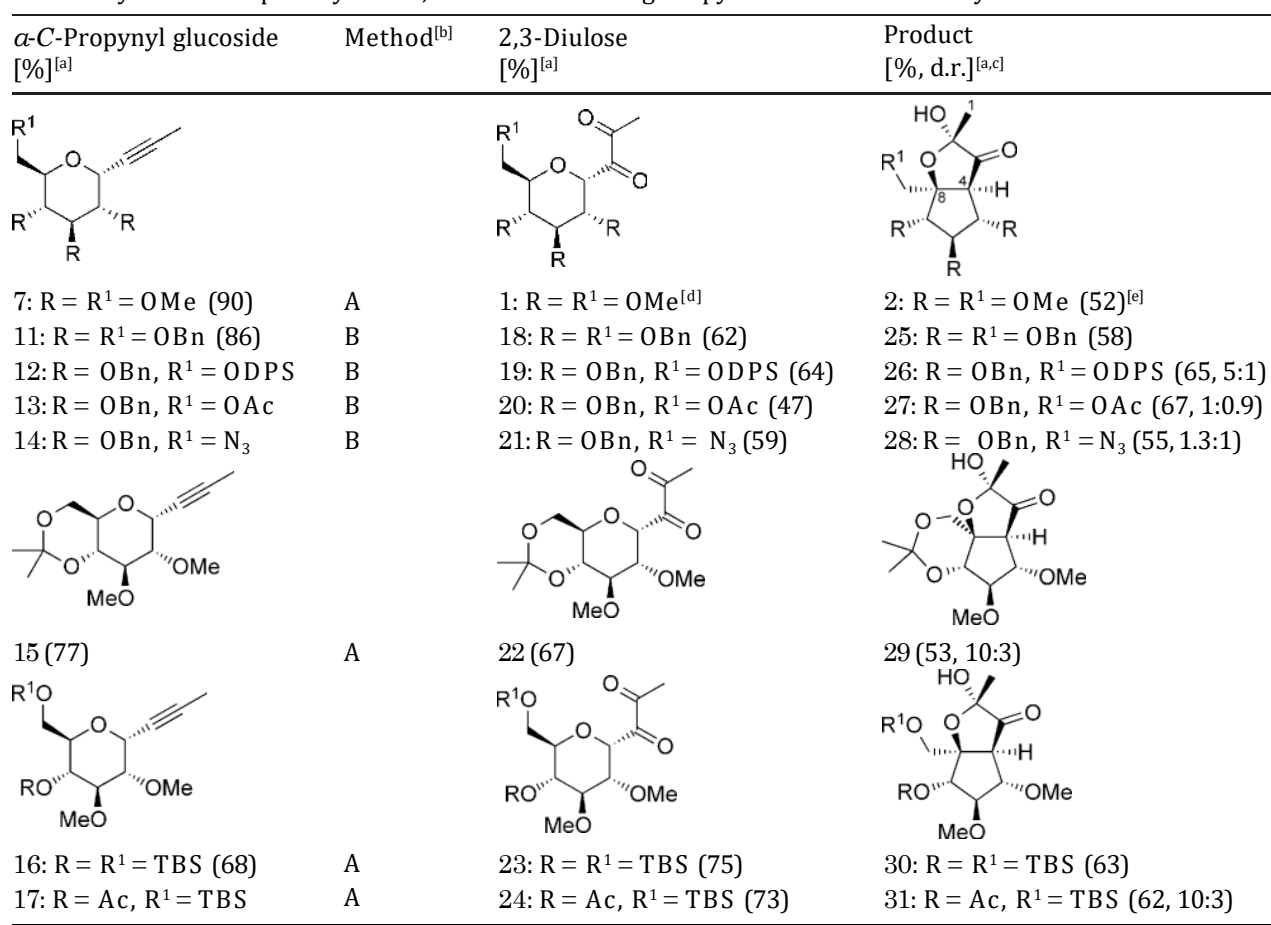

[a] Yield of the product isolated by using chromatography. [b] Method $\mathrm{A}: \mathrm{O}_{3}$ (excess), $\mathrm{CH}_{2} \mathrm{Cl}_{2},-788 \mathrm{C}, 2 \mathrm{~h}$, then $\mathrm{Me}_{2} \mathrm{~S}$ (2.1 equiv), room temperature, dark. Method B: $\mathrm{NaIO}_{4}$ (4 equiv), $\mathrm{RuO}_{2} \cdot x \mathrm{H}_{2} \mathrm{O}$ (8.8 mg), $\mathrm{CCl}_{4}$ / $\mathrm{CH}_{3} \mathrm{CN} / \mathrm{H}_{2} \mathrm{O}$ (1:1:1.5), room temperature, dark. [c] Hemiketal diastereomeric ratio, major isomer shown. [d] Crude product of high purity but could not be purified on chromatography over silica. [e] Yield over three steps. D P S = tert-butyldiphenylsilyl, T BS = tert-butyldimethylsilyl. tion of 1 in $\left[D_{6}\right]$ benzene containing the triplet quencher pyrene (13 equiv) was irradiated at $30 \mathrm{8C}$ for $12 \mathrm{~h}$. The photoreaction was completely inhibited, confirming that the hydrogen abstraction occurs mainly from the triplet state since cleavage products promoted by the singlet biradical were not detected. Compound 2 is a crystalline solid whose structure and stereochemistry was determined by extensive $1 \mathrm{D}$ and $2 \mathrm{D}$ NMR studies and confirmed by X-ray crystallographic analysis. ${ }^{[23]}$ In particular, the ${ }^{3} J_{\mathrm{H} 4, \mathrm{H} 5}=$ $4.7 \mathrm{~Hz}$ (calcd 4.1 Hz), HMBC correlations of $\mathrm{H} 4$ to $\mathrm{C} 8$ and C9, and NOE interactions between the hydroxyl proton and $\mathrm{H} 4$ and $\mathrm{H} 9$ as well as interactions between 1-Me and $\mathrm{H} 5$ and H7 accounted for the b-syn cyclization and the $S$ stereochemistry of the hemiketal. Due to the flexibility of the cyclopentane, weighted average ${ }^{3} J_{\mathrm{H}, \mathrm{H}}$ for the ring protons were calculated for the more popu- 
lated conformers as determined by pseudorotational analysis. ${ }^{[24]}$ Two conformers were obtained with pseudorotational phase angle ( $P$; north- and south-type, $P_{\mathrm{N}} P_{\mathrm{S}}$, respectively) and populations, as indicated: $E_{6}\left(P_{\mathrm{N}}=346.48,60 \%\right)$ and ${ }^{6} T_{7} / E_{7}\left(P_{\mathrm{S}}=189.18,40 \%\right.$; see Table $2 \mathrm{~S}$ and Figure $1 \mathrm{~S}$ in the Supporting Information for details). The more stable conformer is in close agreement with the ring conformation in the solid state: ${ }^{7} T_{6}\left(P_{\mathrm{N}}=358.5\right.$ 8). A small but significant ${ }^{4} J_{\mathrm{H} 5 \mathrm{H} 7}$ coupling (ca. $1 \mathrm{~Hz}$ ) can be detected, which is more consistent with the ${ }^{6} T_{7} / E_{7}$ conformation where both hydro- gens are pseudoequatorial in a W-type planar arrangement. The X-ray crystallographic structure indicated that the con- figuration of the hemiketal is stabilized by an intramolecular sevenmembered hydrogen bond between the hydroxyl group and the oxygen atom at $\mathrm{C} 9$. The reaction was moni- tored by ${ }^{1} \mathrm{H}$ NMR spectroscopy but we were unable to detect any photoenol II under these reaction conditions (30 8C); nevertheless, at $08 \mathrm{C}$ a doublet at $\mathrm{d}=5.56 \mathrm{ppm}(J=9 \mathrm{~Hz})$ and a broad singlet exchangeable with $\mathrm{D}_{2} \mathrm{O}$ at $\mathrm{d}=$

$6.73 \mathrm{ppm}$ assignable to the transient photoenol could be observed. ${ }^{[25]}$ Upon warming to room temperature these signals rapidly disappeared completely, the cyclized product 1 being the only one observable after a few minutes.

Unfortunately, the photolysis of phenyl diulose 9 failed completely to undergo the desired 1,5-HAT, instead providing the photoenol III as the only intermediate detectable by ${ }^{1} \mathrm{H}$ NMR spectroscopy (Scheme 2). ${ }^{[25,26]}$ This intermediate could not be isolated and was rapidly oxidized during the work-up, presumably by the ambient oxygen, ultimately leading to the degraded lactone 10 in moderate yield. A possible inversion of the pyranose ring in the diketone was discarded by the coupling con-

stants, which are
consistent with a $C_{4}$ confoubtedly tion.

The other 2,3-diuloses belonging to d-glucopyranose series 18-24 (Table 1) with different substituents at $\mathrm{C} 7$ and C9, vicinal carbon atoms in the INHAT, were synthesized to determine the influence of steric and electronic effects in both the reactivity of the diradi- cal intermediate I and the diastereoselectivity of the aldol reaction. The reactions involved in this transformation do not seem to be influenced greatly by the steric bulkiness or electronegativity of the protecting groups, similar yields being obtained and only products from OH-b-syn aldol cyclization being observed. The somewhat lower yields obtained in the synthesis of 2 and 29 could be more related with the instability of the diulose itself.

The coupling constant ${ }^{3} J_{\mathrm{H} 4, \mathrm{H} 5}$ proved to be diagnostic in distinguishing between the $\mathrm{OH}-b$-syn and $\mathrm{OH}-a-s y n$ aldol cyclization (see below, ${ }^{3} J_{\mathrm{H} 4 a, \mathrm{H} 5 b}=2-4 \mathrm{~Hz}$ is much smaller than ${ }^{3} \boldsymbol{J}_{\mathrm{H} 4 b, \mathrm{H} 5 b}=9-10 \mathrm{~Hz}$ ). However, due to the flexibility of the cyclopentane ring the stereochemistries have been confirmed further by nuclear Overhauser effect spectroscopy (NOESY) studies on minimized structures. In those compounds 2 and 29-31 in which the experimental ring coupling constants could be unequivocally determined, a conformational study by pseudorotational analysis has been performed. Two relatively narrow pseudorotational ranges are preferred for these d-glucose-derived cyclopentanes. In the North-type conformations $P$ ranges from 311 to $3468\left({ }^{5} E /{ }^{5} T_{6}\right.$ to $E_{6}$ ) were observed, whereas $P$ values of $106-1898\left({ }^{4} T_{5}\right.$ to ${ }^{6} T_{7}$ ) were found for the conformations positioned in the South region of the pseudorotational wheel (See Table $2 \mathrm{~S}$ and Figure $1 \mathrm{~S}$ of the Supporting Information for details).

The next series of reactions explored the photolysis of deoxy-2,3-diuloses 38-43 as described in Table 2. The reactions proceeded analogously to the previously mentioned examples in terms of yield and diastereoselectivity except for 5-deoxy-diulose 38, which gave a 1:1 diastereomeric mix- ture of $\mathrm{OH}-b$-syn 44 and $\mathrm{OH}-a$-syn 45 aldol cyclization products after heating for $5 \mathrm{~h}$ at $60 \mathrm{8C}$. The photolysis mix- ture was subjected to acetylation thus allowing for a partial chromatographic separation of acetates 44 and 45 .

The $0 \mathrm{H}-b$-syn cyclization structure was assigned to 44 by the ${ }^{3} J_{\mathrm{H} 4 a, \mathrm{H} 5 b}=4.4 \mathrm{~Hz}$ and substantiated by the observation of NOESY correlations between 1-Me and H7, and H4 and H6. Moreover, a larger ${ }^{3} J_{\mathrm{H} 4, \mathrm{H} 5}=9,8 \mathrm{~Hz}$ coupling constant
Table 2. Synthesis and photolysis of mono- and di-deoxy-2,3-diuloses in pyranose form.

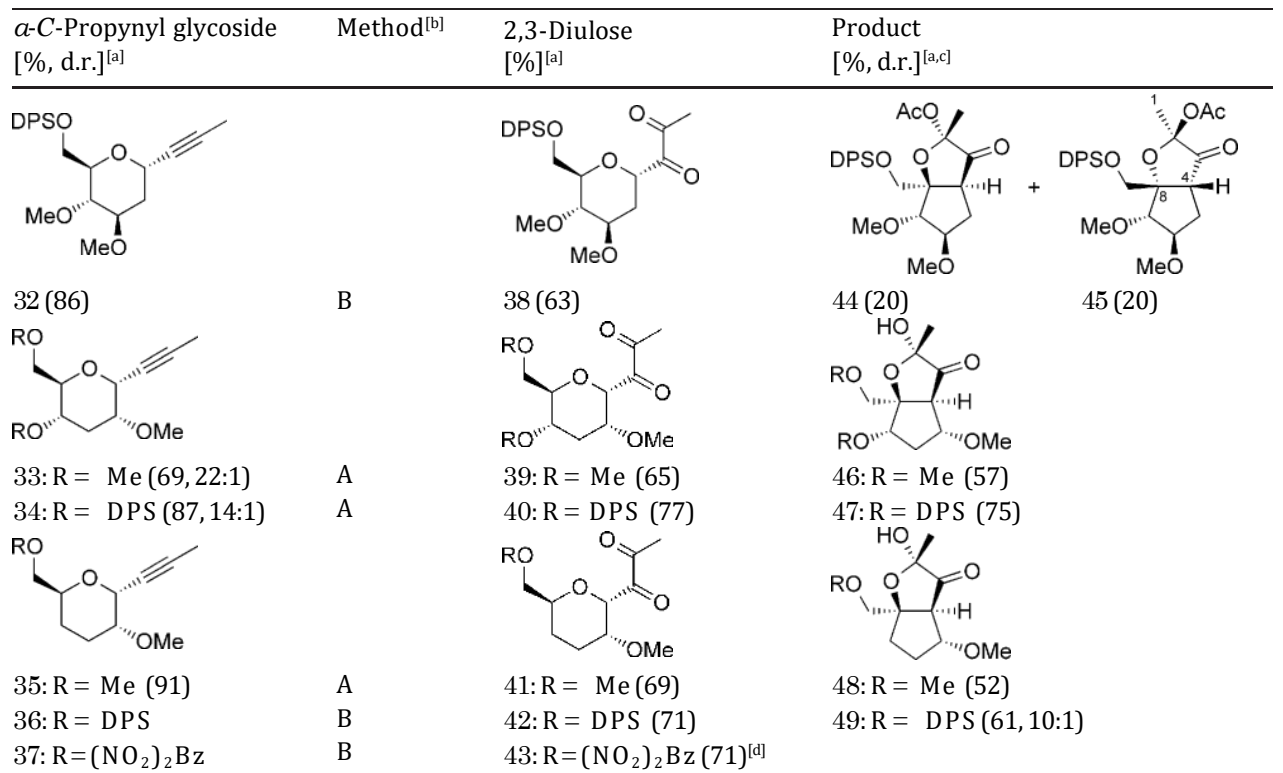

[a] Yield of the product isolated by using chromatography. [b] Method A: $\mathrm{O}_{3}$ (excess), $\mathrm{CH}_{2} \mathrm{Cl}_{2},-788 \mathrm{C}, 2 \mathrm{~h}$, then $\mathrm{Me}_{2} \mathrm{~S}$ (2.1 equiv), dark. Method B: $\mathrm{NaIO}_{4}$ (4 equiv), $\mathrm{RuO}_{2} \cdot x \mathrm{H}_{2} \mathrm{O}$ (8.8 mg), $\mathrm{CCl}_{4} / \mathrm{CH}_{3} \mathrm{CN}_{/} \mathrm{H}_{2} \mathrm{O}$ (1:1:1.5), room temperature, dark. [c] Hemiketal diastereomeric ratio, major isomer shown. [d] Thermolysis of 43 was never realized. $\mathrm{Bz}=$ benzyl. 
and NOESY correlations between 1-Me and H6, and H4 and $\mathrm{H} 7$ were consistent with an $\mathrm{OH}-a$-syn cyclization struc- ture for the isomer 45 .

The most unexpected results were found for the deoxy compounds 40-43, which were transformed into the intermediate photoenols 50-53 upon irradiation at 15-35 8C (Scheme 3). Compounds 50-53, which are surprisingly stable
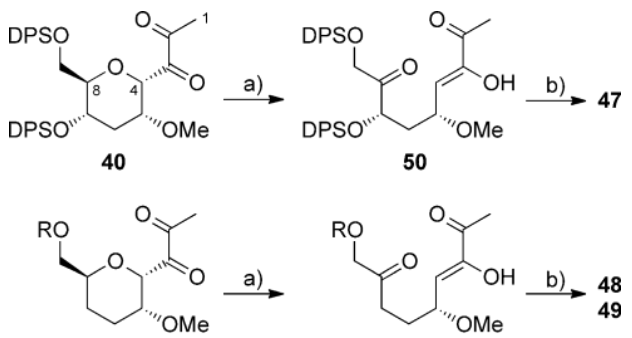

41: $R=M e$

42: $R=D P S$

43: $\mathrm{R}=\left(\mathrm{NO}_{2}\right)_{2} \mathrm{Bz}$

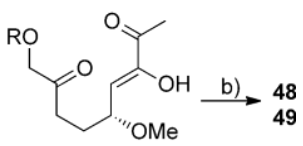

51: $\mathrm{R}=\mathrm{Me}$

52: $\mathrm{R}=\mathrm{DPS}$

53: $\mathrm{R}=\left(\mathrm{NO}_{2}\right)_{2} \mathrm{Bz}$

Scheme 3. Synthesis and aldol reactions of stable photoenols 50-53. a) Daylight-lamp irradiation, $\mathrm{C} \mathrm{B}_{6}$; b) heat at $408 \mathrm{C}$ for the synthesis of $47(78 \%)$ from 50 , and at $60 \mathrm{C}$ for the synthesis of $48(52 \%)$ and 49 $(61 \%)$ from 51 and 52 , respectively. Thermolysis of 53 was never realiz- ed.

at room temperature for extended periods, could be isolated without significant contamination by either the starting material or the respective cyclized product. The oily crude residues did not withstand chromatographic purification over silica gel or alumina but were pure enough to allow complete analytical and spectroscopic characterization. A nuclear Overhauser effect (NOE) interaction between the methyl ketone and the vinyl proton is indicative of the $Z$ configuration for the double bond as shown in Scheme 3. Photoenol 50 obtained by photolysis of 40 at $25 \mathrm{8C}$ cyclized upon heat- ing at $408 \mathrm{C}$ in light-protected benzene solution for $8 \mathrm{~h}$ to afford carbocycle 47 . Photoenols 51 and 52, which are ther- mally more stable, were cy-

clized in the dark at $60 \mathrm{8C}$ to give carbocycles 48 and 49 , respectively.

These thermal experiments, performed with strict exclusion of light, confirm that the aldol reaction proceeded with a high degree of diastereoselectivity, resulting in the exclusive formation of syn-aldols. We did not find any anti-aldol product that (having the $a$-diketone moiety unprotected) could have been destroyed by secondary photolysis in the prior illuminated experiments. Several attempts to obtain suitable crystals of the photoenol for X-ray crystallographic analysis by derivatiza- tion of the primary alcohol (e.g., 3,5-dinitrobenzoate 53) were unsuccessful.

We next prepared 2,3-diuloses of the d-manno 58 and dgalacto 59-61 series to evaluate the influence of the C5 and C7 stereochemistry in the diastereoselectivity of the aldol reaction (Table 3). We anticipated on the basis of the previous experiment with 5-deoxy-diulose 38 (Table 2) that the aldol cyclization could show a marked dependence on the stereochemistry of the substituents at $\mathrm{C} 5$. This was indeed the case; the photolysis of 58 at $30 \mathrm{8C}$ and subsequent heat- ing at 50 8C led exclusively to the formation of the a-syn isomer 62 , albeit in moderate yield. The crude reaction had to be acetylated to facilitate the purification of 62 from a complex reaction mixture. In the mannose skeleton, an al- ternative 1,5-HAT reaction of the $\mathrm{H} 5 \mathrm{a}$ proton by the exter- nal carbonyl under classical Norrish-Yang conditions is pos- sible. Thus, competitive abstraction of H8a and H5a may account for the somewhat lower efficiency of this process but we have been unable to isolate or identify any side products to confirm this hypothesis.

The ${ }^{1} \mathrm{H}$ NMR spectrum of the permethylated d-galactose model 59 exhibits ring coupling constants that strongly point to a ${ }^{4} C_{7}$ chair conformation. It was therefore not very surprising that the photochemical reaction gave 2,3,4,6-tetra- $O$ methyl- $a$-d-galactono-1,5-lactone as the only detectable product in $80 \%$ yield. Since in this geometric arrangement the $a$-diketone tether and the H8 $a$ are in a 1,3-diequatorial disposition, the 1,5-HAT by the internal carbonyl is impossible. The excited carbonyl preferentially abstracts the axial $\mathrm{H} 4 b$ to give a 3,4-photoenol intermediate (such as III, Scheme 2) which is subsequently and presumably degraded to the lactone by adventitious oxygen during the workup.

The tert-butyldiphenylsilyl (DPS) derivative 60 was then synthesized in the hope that an extremely bulky protecting group at the 9-primary alcohol causes a flip of the $C$-glycoside conformation to the ${ }^{7} C_{4}$ chair. ${ }^{[27]}$ Fortunately, the ring coupling constants $\left({ }^{3} J_{\mathrm{H} 4, \mathrm{H} 5}=6.9,{ }^{3} J_{\mathrm{H} 5, \mathrm{H} 6}=8.5\right.$, and ${ }^{3} J_{\mathrm{H} 6, \mathrm{H} 7}=$
Table 3. Synthesis and photolysis of 2,3-diuloses with $a$-d-manno- and $a$-d-galacto-pyranose stereochemistry.

a-C-Propynyl
glycoside [\%, d.r. $]^{[a]}$

[a] Yield of the product isolated by using chromatography. [b] Method $\mathrm{A}: \mathrm{O}_{3}$ (excess), $\mathrm{CH}_{2} \mathrm{Cl}_{2},-788 \mathrm{C}, 2 \mathrm{~h}$, then $\mathrm{Me}_{2} \mathrm{~S}$ (2.1 equiv), dark. Method B: $\mathrm{NaIO}_{4}$ (4 equiv), $\mathrm{RuO}_{2} \times \mathrm{H}_{2} \mathrm{O}$ (8.8 mg), $\mathrm{CCl}_{4} / \mathrm{CH}_{3} \mathrm{CN} / \mathrm{H}_{2} \mathrm{O}$ (1:1:1.5), room temperature, dark. [c] Irradiation of 59 led to 2,3,4,6-tetra- $O$-methyl-d-galactono-1,5-lactone exclusively. 
$2.6 \mathrm{~Hz}$ ) confirmed this assumption and the photolysis-heating process on 60 afforded a mixture of two diastereomeric cyclopentitols, which was acetylated and separated into 63 and 64 after careful chromatography (d.r. 1:2). The intermediate photoenol could be fully characterized by monitoring the reaction by using ${ }^{1} \mathrm{H}$ NMR spectroscopy and subsequent heating at $708 \mathrm{C}$ was necessary to accomplish the aldol cyclization.

The structure of 63 and 64 as b-syn and a-syn cyclization isomers, respectively, was assigned on the previously commented basis: The ${ }^{3} J_{\mathrm{H} 4, \mathrm{H} 5}$ coupling constant, which is smaller for the $b$-syn isomer $\left({ }^{3} J_{\mathrm{H} 4 a, \mathrm{H} 5 b}=2.9 \mathrm{~Hz}\right.$ for 63 versus ${ }^{3} J_{\mathrm{H} 4 b, \mathrm{H} 5 b}=$ $9.5 \mathrm{~Hz}$ for 64), and NOESY experiments. To calcu- late the ring coupling constants and $\mathrm{NOE}$ interaction the most populated conformer was determined to be ${ }^{7} T_{6}$ for both isomers $(P=6.9$ for 63 and $355.8 \mathrm{8}$ for 64$)$ by using pseudorotational analysis (see the Supporting Information for details). Similar results were observed in the reaction of 9acetyl derivative 61: a 1:3 mixture of 65 and 66 was ob- tained in good overall yield (76\%), in which the $a$-syn isomer was also the major product.

In addition, a number of octo-2,3-diuloses of the pentofuranose series of carbohydrates with d-ribo (70 a and $71 \mathrm{~b})$ and d-lyxo (72) stereochemistries were next synthesized to study the influence of the furanose ring conformation in the 1,5 HAT reaction (Table 4). The photolysis of the d-ribo derivative 70 a led to the lactone 73 in moderate yield, presumably by oxidation of the photoenol intermediate 74 a, as previously observed in diketones 9 and 59 in which photoenolization competes with photoelimination. ${ }^{[26]}$ The most populated conformer of 70 a has a phase angle of $P=2568$

Table 4. Synthesis and photolysis of 2,3-diuloses with $a$-d-ribo- and $a$-dlyxofuranose stereochemistry.

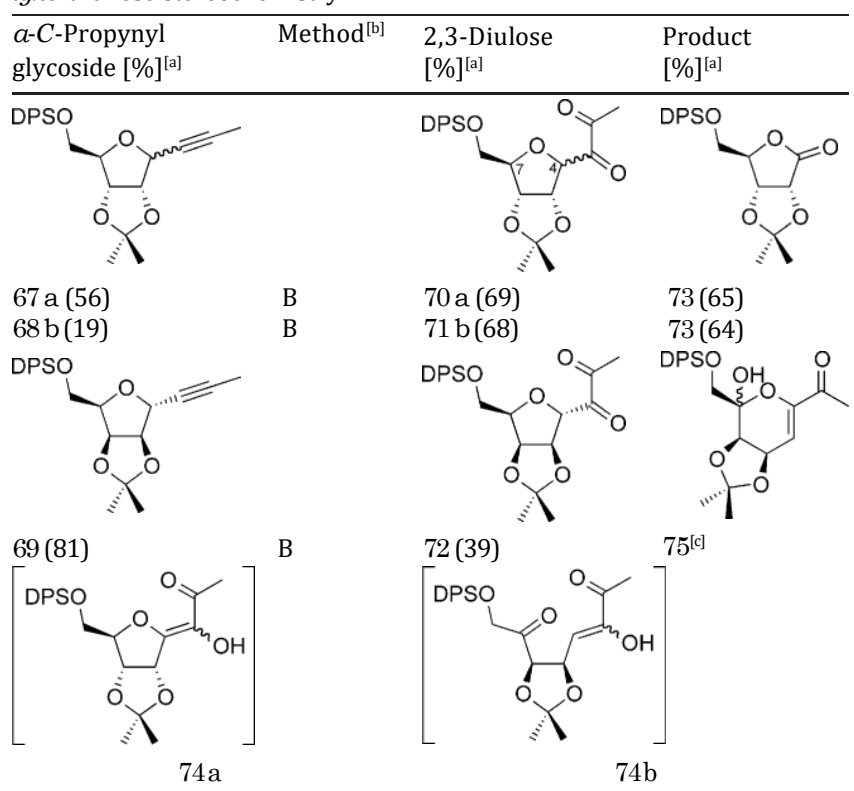

[a] Yield of the product isolated by using chromatography. [b] Meth- od B: $\mathrm{NaIO}_{4}$ (4 equiv), $\mathrm{RuO}_{2} \cdot x \mathrm{H}_{2} \mathrm{O}$ (8.8 mg), $\mathrm{CCl}_{4} / \mathrm{CH}_{3} \mathrm{CN} / \mathrm{H}_{2} \mathrm{O}$ (1:1:1.5), room temperature, dark. [c] The product was chromatographically unsta- ble. $\left({ }^{7} T_{0}\right)$, wherein the $a$-diketone tether and the hydrogen at C7 lie in a syn-1,3-pseudo-diequatorial relationship. The distance between the oxygen at C3 and the extractable hydrogen at $\mathrm{H} 7$ on the minimum energy conformer was calculated as indicative of the 1,5-HAT feasibility. Since it is generally accepted that a narrow range of distances of approx. 2.5-

$3.0 \AA$ are required for the reaction to take place, the obtained value of $d_{\mathrm{C} 3=0 \cdots \mathrm{H} 7}=4.7 \AA$ precludes any photochemical hydrogen abstraction. ${ }^{[28]}$

The abstraction at $\mathrm{H} 7$ is not geometrically feasible in the dribo isomer $71 \mathrm{~b}$, but the abstraction of $\mathrm{H} 5$ by the excited external carbonyl to result in a Yang photocyclization is worthy of consideration. Notwithstanding, the photolysis of $71 \mathrm{~b}$ gave the same lactone 73 in similar yield, demonstrating that in these sugar derivatives photoenolization also competes favorably with the Yang cyclization. ${ }^{[26]}$ In contrast to dribo derivative $70 \mathrm{a}$, the furanose ring of the isomeric $\mathrm{d}$ - lyxo 72 adopts a conformation ${ }^{\circ} E(P=868)$ with the $a$-dike- tone and the $\mathrm{H} 7$ in a syn-1,3-pseudo-diaxial relationship. In the global minimum the INHAT distance now possesses an ideal value of $2.53 \AA$ and the photoelimination proceeded smoothly to give 75 presumably by intramolecular hemiketalization of the intermediate photoenol 74 b. $a, b$-Unsaturated ketone 75 could not withstand chromatographic purification but the crude reaction residue was pure enough to allow complete analytical and spectroscopic characteriza- tion.

As commented previously, we propose a sequential mechanism for this transformation with two clearly differentiated steps (Scheme 1): A Norrish type II photoelimination of the a-diketone that opens the pyranose ring leading to the acyclic photoenol II, which subsequently undergoes an intramolecular aldol cyclization reaction. The photoelimination implies an 1,5-HAT of the H8 by the excited innermost carbonyl of the $a$-diketone, followed by $b$-fragmentation of the 1,4-biradical intermediate I. The Norrish type II photoelimination, although very common in monoketones, ${ }^{[1]]}$ is extremely rare in $a$-diketones, in which only a few synthetically irrelevant examples are documented. ${ }^{[29]}$ This reaction has been postulated to occur through a hydrogen abstraction by the innermost carbonyl, which is otherwise completely unreactive from the photochemical point of view. ${ }^{[30]}$

The distance between the carbonyl oxygen and the $g$-hydrogen associated with the transition state of the 1,5-HAT has been determined in monoketones to have a critical value of around $2.7 \AA^{\left[{ }^{[31]}\right.}$ It is not surprising, therefore, that the hydrogen abstraction is strongly dependent on the pyra- nose ring conformation of the $a$ - $C$-glycoside: favored when a ${ }^{7} C_{4}$ chair is adopted and highly disfavored in ${ }^{4} C_{7}$ arrange- ments.

As far as we are aware, the uncatalyzed thermal syn aldol cyclization in which a $Z$-photoenol (such as II, Scheme 1) acts as a preformed enolate is an unprecedented process. ${ }^{[32]}$ According to Baldwin rules $^{[33]}$ the 5-(enolexo)-exo-trig ring closure involved is stereoelectronically favored and may be considered to be a new intramolecular carbonyl-ene cyclization, in which the carbonyl group and the enol can act as 


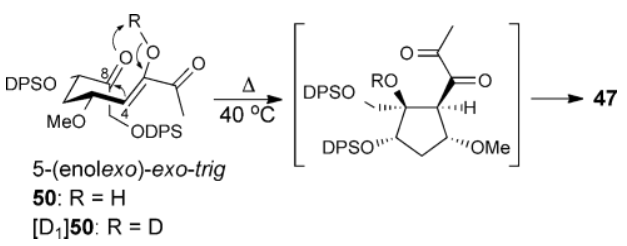

Scheme 4. Thermal kinetic studies with 50 and $\left[D_{1}\right] 50$.

enophile and ene unit, respectively (Scheme 4). ${ }^{[34]}$ The rearrangement is accompanied by migration of the double bond and a 1,5-shift of the enolic hydrogen. ${ }^{[35]}$ These thermal reactions, studied in detail with more stable deoxy-photoenols 50-52 (Scheme 3), proceeded with low activation energy (4060 8C). Comparatively, the uncatalyzed carbonyl-ene processes usually require much higher temperatures. ${ }^{[36]}$ Surprisingly, the cyclization temperature may be even lower in more substituted pyranose frameworks; for example, permethylated diketone 1 was transformed into 2 by irradiation at 0 8C for $12 \mathrm{~h}$ in $55 \%$ yield. In contrast, the dideoxy photoen- ol 51 is stable at room temperature and requires $60 \mathrm{8C}$ for complete cyclization. This striking steric acceleration effect has been previously observed in an uncatalyzed ene reaction. ${ }^{[37]}$ Mechanistic studies of the ene-reaction have demonstrated that a large deuterium kinetic isotope effect (KIE; $k_{\mathrm{H}} / k_{\mathrm{D}}>2$ ) indicates that the INHAT is the rate-limiting step and has often been taken as evidence of a concerted mechanism. ${ }^{[38]}$ Taking advantage of the ready deuteration of the photoenol, we decided to determine the intramolecular hydrogen-deuterium isotope effect for the thermal reaction of photoenol 50 (Scheme 4). Photoenol 50 was chosen because its cyclization into product 47 proceeded smoothly at a moderate temperature and rate. The value of the reaction rate constants for undeuterated $50\left(k_{\mathrm{H}}\right)$ and deuterated $\left[\mathrm{D}_{1}\right] 50$ $\left(k_{\mathrm{D}}\right)$ were measured by scanning at regular intervals $\left[D_{6}\right]$ benzene dissolved samples placed in a preheated (40 8C) NMR probe and monitoring integral data for the vinylic proton. The value of KIE obtained $\left(k_{\mathrm{H}} / k_{\mathrm{D}}=3.7\right)$ appears to indicate a concerted pericylic mechanism.

Models accounting for the observed relative stereochemistry invoke the intermediacy of a $Z$ enolate, intramolecular hydrogen-bonding between the $\mathrm{OH}$ and the carbonyl, and a Zimmerman-Traxler-type transition state, which has been frequently used for catalytic intramolecular aldol reactions (Figure 1). ${ }^{[10,39]}$ The presence of this hydrogen bond is anticipated to confer additional stability and may help to maintain the five-membered transition state in a prearranged conformation for cyclization. As expected from the models of Figure 1,

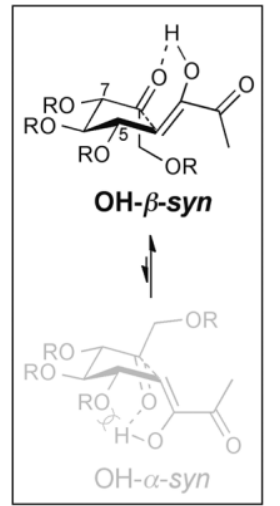

D-gluco major isomer is highlighted. the major stereoisomer is that in which the two newly created stereogenic centers are cis to each other. This is probably very important for the overall yield of the reaction because it permits the internal acetalization of the regenerated $a$-diketone moiety avoiding secondary photolysis. ${ }^{[40]}$

The steric influence of the adjacent substituents at C5 and C7 in the stereochemical outcome can also be easily inferred from these models (Figure 1). Interactions with the $5 a$-equatorial substituent in d-gluco $(1,18-24$, Table 1), 6-deoxy-dgluco (39-40, Table 2), and 6,7-dideoxy-d-gluco (41-42, Table 2) derivatives afforded exclusively $\mathrm{OH}-b$-syn cycliza- tions. The influence of this substituent is confirmed in the 5- deoxyd-gluco derivative (38), in which an equimolecular mixture of $\mathrm{OH}-b$-syn and $\mathrm{OH}-a$-syn isomers was formed. In- version of configuration at C5 in d-manno derivative (58) entails a more crowded $b$-face of the transition-state (TS) ring with exclusive formation of the $\mathrm{OH}-\mathrm{a}$-syn isomer. This effect is also observed in d-galacto derivatives (59-61), thus leading preferentially to a majority of $\mathrm{OH}-a$-syn cyclization products.

Degradation of the masked $a$-diketones could be realized under oxidative conditions by using two different methodologies: With periodic acid in methanol (Method $\mathrm{A})^{[41]}$ or by $b$ fragmentation of the alkoxyl radical, generated from the hemiketal alcohol by reaction with the $\mathrm{PhI}(\mathrm{OAc})_{2} / \mathrm{I}_{2}$ system (Method B) ${ }^{[42]}$ as depicted in Table 5. With method A the yields were excellent with substrates bearing very stable protective groups (e.g., methyl ethers, Table 5, entries 1 and 4) but decreased substantially with easily oxidized (e.g., benzyl ethers, entry 2) or acid-sensitive groups (e.g., silyl ethers, entries 3 and 7). Method B, however, turned out to be more efficient with substrates possessing these sensitive groups, for example, disilyl compound 47 (Table 5, entry 7). The efficiency of both methods is similar with stable protective groups (entry 5). Another observation that emerges from these experiments is that methylation with diazomethane, either of the crude or purified acid, considerably decreases the global yield (compare method A, Table 5, entries 4 and 5 or method B, entries 6 and 7).

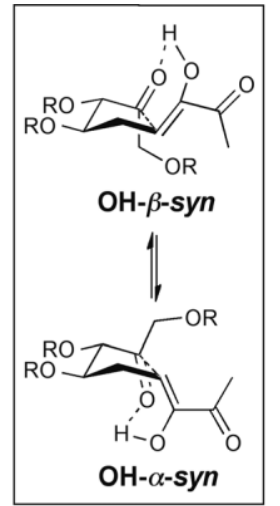

5-deoxy-D-gluco

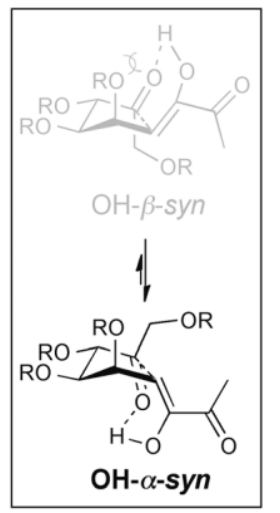

D-manno

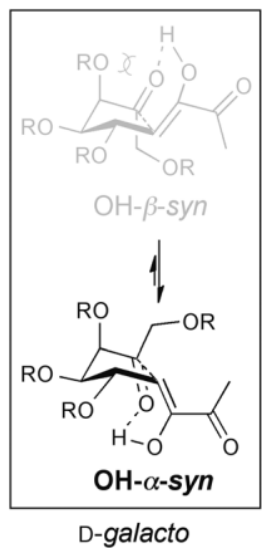

D-galacto

Figure 1. Proposed models for the stereochemical course of the aldol reaction. The transition state (TS) of the 


\begin{tabular}{lll}
\hline Entry Substrate & $\begin{array}{l}\text { Method } \\
{[\%]^{[a, b]}}\end{array}$ & Product \\
\hline & & \\
& &
\end{tabular}

[a] Method A: $\mathrm{H}_{5} \mathrm{IO}_{6} \cdot 2 \mathrm{H}_{2} \mathrm{O}, \mathrm{MeOH}$, room temperature; Method B: PhI$(\mathrm{OAc})_{2}, \mathrm{I}_{2}, h \mathrm{n}$, room temperature. [b] Yield of the product isolated by using chromatography. [c] Methylation with diazomethane.
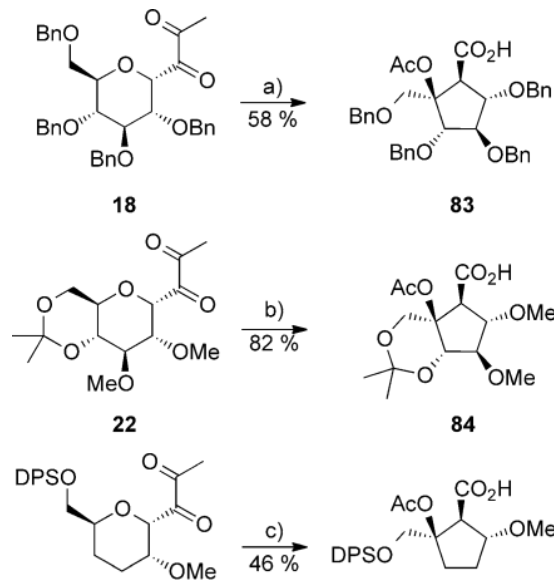

83

$$
42
$$
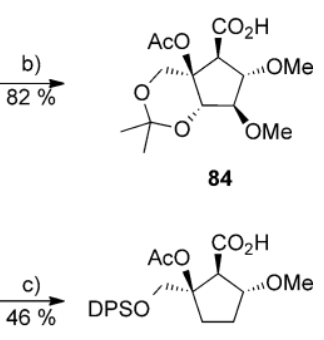

85

Scheme 5. Synthesis of cyclopentitols from 2,3-diuloses in a one-pot, threestep sequence. a) Daylight-lamp irradiation, $\mathrm{C}_{6} \mathrm{D}_{6}, 308 \mathrm{C}, 4 \mathrm{~h}$, then $\mathrm{H}_{5} \mathrm{IO}_{6} \cdot 2 \mathrm{H}_{2} \mathrm{O}$, $\mathrm{MeOH}$, room temperature, $3 \mathrm{~h}$; b) daylight-lamp irradiation, $\mathrm{C}_{6} \mathrm{D}_{6}, 36-408 \mathrm{C}, 2.5 \mathrm{~h}$, then $\mathrm{PhI}(\mathrm{OAc})_{2}, \mathrm{I}_{2}, \mathrm{hn}$, room temperature, 40 min; c) daylight-lamp irradiation, $\mathrm{C}_{6} \mathrm{D}_{6}, 36-40 \mathrm{8C}, 5 \mathrm{~h}$, then $\mathrm{H}_{5} \mathrm{IO}_{6} \cdot 2 \mathrm{H}_{2} \mathrm{O}$, $\mathrm{MeOH}$, room temperature, $3 \mathrm{~h}$. Product isolated by using silica gel chromatography over three steps.

Another set of experiments in which the crude residue of the photolysis-heating reaction is directly treated with the oxidant reagents is summarized in Scheme 5. $a$-Diketones 18, 22 , and 42 were converted into pure cyclopentanes $83-85$, respectively, in good overall yield by using this one-pot, threestep process.

To confirm the structure of the cyclopentane derivatives and to obtain information on their chemical reactivity, a series of short chemical transformations were performed (Scheme 6). The hydrolysis and subsequent methylation with diazomethane of the previously prepared acid 83 af- forded the $a$-hydroxyester 86 whose relative disposition was
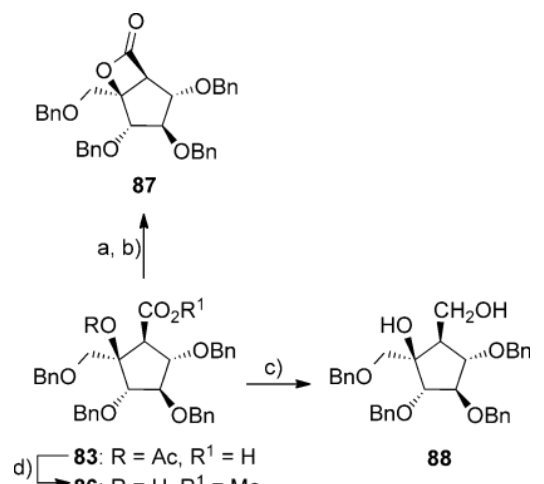

d) $\longrightarrow$ 86: $R=H_{1} R^{1}=M e$

88

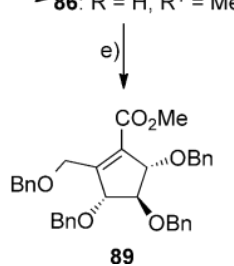

Scheme 6. Some initial experiments on the reactivity of cyclopentitols. a) $\mathrm{KOH}, \mathrm{MeOH}$, room temperature, $24 \mathrm{~h}$; b) $\mathrm{PhSO}_{2} \mathrm{Cl}, \mathrm{Py}, 08 \mathrm{C}, 14 \mathrm{~h}, 73 \%$, two-step; c) $\mathrm{LiAlH}_{4}, \mathrm{THF}, 0 \mathrm{8C} 4 \mathrm{~h}, 78 \%$; d) $\mathrm{KOH}, \mathrm{MeOH}$, room temperature, $24 \mathrm{~h}$, then $\mathrm{CH}_{2} \mathrm{~N}_{2}, 99 \%$; e) $\mathrm{Tf}_{2} \mathrm{O}, \mathrm{CH}_{2} \mathrm{Cl}_{2}, \mathrm{Py}, 08 \mathrm{C}, 24 \mathrm{~h}$, $93 \%$.

confirmed by dehydration to give the $a, b$-unsaturated ester 89 . The $b$-lactone 87 was prepared also using acid 83 as starting material, by treatment with benzenesulfonyl chloride in pyridine after hydrolysis of the acetyl group. The formation of 87 confirms that it does indeed possess the synhydroxy carboxylate arrangement generated during the aldol reaction. Finally, the reduction of 83 with an excess of $\mathrm{LiAlH}_{4}$ afforded the diol 88 spectroscopically identical to the one synthesized by Chiara et al. ${ }^{[43]}$

\section{Conclusion}

The new sequential reaction described herein results in the direct formation of densely polyfunctionalized cyclopentitols by ring contraction of hexopyranose carbohydrates. It is worth noting that the reaction proceeds efficiently in a one- pot twostep process with no reagents other than light and heat. The easy accessibility of pyranose sugars of various configurations and the possibility of prior structural modifi- cations illustrate the potential of this methodology for the preparation of chiral synthons of cyclopentanoid natural products. To the best of our knowledge, this is the first syn- thetic application of the Norrish type II photoelimination in- itiated by the innermost carbonyl of a $a$-diketone. The im- portance of this reaction is that it uniquely generates an acy- clic keto-enol system, which is ideally suited for a subse- quent 5-(enolexo)exo-trig intramolecular aldol cyclization. This thermal uncatalyzed aldol reaction in which a $Z$ photo- enol acts as a preformed enolate is also unprecedented. The stereochemistry of the cyclization is strongly dependent on the configuration of the sugar-ring substituents; for example, 
a d-gluco arrangement of the starting material leads exclusively to $\mathrm{OH}-b$-syn products, whereas an inverted $\mathrm{OH}-a$-syn cyclization is observed for the d-manno series of carbohydrates.

\section{Experimental Section}

General procedure for the oxidation of C-propynyl glycosides to a-diketones: Method A: A solution of $C$-propynyl glycoside (1 equiv) in dry $\mathrm{CH}_{2} \mathrm{Cl}_{2}(20 \mathrm{~mL})$ was cooled to $-788 \mathrm{C}$, ozone was introduced into the solution and the reaction monitored by TLC. Then, nitrogen was bubbled through the solution to expel excess of ozone, dimethyl sulfide $(2-10$ equiv) was added and the mixture was gradually warmed to room temperature and stirred at this temperature for the specified time in the dark. The mixture was concentrated under reduced pressure to give a yellow residue that, in most cases, can be purified by rapid silica gel chromatography (hexanes/Et0Ac mixtures) on a column protected from light with aluminum foil, although a significant loss of material was generally observed.

Method $B$ : $\mathrm{NaIO}_{4}$ (3-4 equiv) and $\mathrm{RuO}_{2} \times \mathrm{H}_{2} \mathrm{O}$ (3-12 mg) were addedto a solution of $C$-propynyl glycoside (1 equiv) in $\mathrm{CCl} / \mathrm{CH} \mathrm{CN} / \mathrm{H}_{3}$ (17 $\mathrm{mL}, 1: 1: 1.5)$ and the mixture stirred at room temperature for the specified time in the dark. The mixture was diluted with $\mathrm{CH} \mathrm{Cl}$, dried over anhydrous $\mathrm{Na}_{2} \mathrm{SO}_{4}$, filtered and concentrated under reduced pres- sure. The residue can be purified by rapid silica gel chromatography (hexanes/EtOAc mixtures) on a column protected from light with aluminum foil, although a significant loss of material was generally observed.

2,6-Anhydro-7,8-dideoxy-1,3,4,5-tetra-O-methyl-8-(trimethylsilyl)-d-glycero-1-gulo-oct-7-ynitol (5): $\mathrm{Bu}_{3} \mathrm{SnC} \div \mathrm{CTMS}(1.49 \mathrm{~g}, 3.86 \mathrm{mmol})$ was added to a solution of $3^{[44]}(538 \mathrm{mg}, 1.93 \mathrm{mmol})$ in dry $\mathrm{CH}_{2} \mathrm{Cl}_{2}(8 \mathrm{~mL})$ containing oven-dried powdered $4 \AA$ molecular sieves $(1.1 \mathrm{~g})$ and stirred at room temperature under nitrogen for $15 \mathrm{~min}$. Trimethylsilyl trifluoromethanesulfonate ( $873 \mathrm{~mL}, 4.83 \mathrm{mmol}$ ) was then added dropwise and the stirring continued for $3 \mathrm{~h}$. The mixture was diluted with $\mathrm{CH}_{2} \mathrm{Cl}_{2}(32 \mathrm{~mL})$, neutralized with $\mathrm{Et}_{3} \mathrm{~N}$, and filtered through a Celite pad. The filtrated was concentrated under reduced pressure and the residue dissolved in EtOAc and washed with an aqueous saturated solution of KF. The organ- ic layer was dried over $\mathrm{Na}_{2} \mathrm{SO}_{4}$ and concentrated under reduced pressure. The residue was purified by silica gel column chromatography (hex-

anes!hexanes/EtOAc 9:1) to give 5 ( $403 \mathrm{mg}, 1.28 \mathrm{mmol}, 66 \%$ ) as an oil: $[\mathrm{a}]_{\mathrm{D}}=+130 \mathrm{~cm}^{3} \mathrm{~g}^{-1} \mathrm{dm}^{-1}\left(\mathrm{c}=3.53\right.$ in $\left.\mathrm{CHCl}_{3}\right) ;{ }^{1} \mathrm{H} \mathrm{NMR}(500 \mathrm{MHz}$, $\left.\mathrm{CDCl}_{3}\right): \mathrm{d}=0.18(\mathrm{~s}, 9 \mathrm{H}), 3.16(\mathrm{dd}, J=9.3,9.8 \mathrm{~Hz}, 1 \mathrm{H}), 3.23(\mathrm{dd}, J=5.6$, $9.5 \mathrm{~Hz}, 1 \mathrm{H}$ ), 3.39 (s, 3H), 3.43 (dd, $J=9.3,9.3 \mathrm{~Hz}, 1 \mathrm{H}$ ), 3.46 (s, 3H), 3.54 (s, $3 \mathrm{H}$ ), 3.57 (dd, $J=10.7,2.3 \mathrm{~Hz}, 1 \mathrm{H}$ ), 3.60 (dd, $J=10.7,3.7 \mathrm{~Hz}, 1 \mathrm{H}$ ), 3.63 (s, $3 \mathrm{H}$ ), 3.82 (ddd, $J=10.1,3.7,2.3 \mathrm{~Hz}, 1 \mathrm{H}$ ), $4.81 \mathrm{ppm}(\mathrm{d}, J=5.6 \mathrm{~Hz}$, $1 \mathrm{H}) ;{ }^{13} \mathrm{C} \mathrm{NMR}\left(100.6 \mathrm{MHz}, \mathrm{CDCl}_{3}\right): \mathrm{d}=-0.1\left(3 \times \mathrm{CH}_{3}\right), 58.5\left(\mathrm{CH}_{3}\right), 59.1$ $\left(\mathrm{CH}_{3}\right), 60.4(\mathrm{CH})_{3} 60.7(\mathrm{CH}), 66.5(\mathrm{CH}), 71.2(\mathrm{CH}), 73_{2} 1(\mathrm{CH}), 79.2$ $(\mathrm{CH}), 80.7(\mathrm{CH}), 84.3(\mathrm{CH}), 94.8(\mathrm{C}), 99.7 \mathrm{ppm}(\mathrm{C})$; IR (film): $\tilde{n}=2931$, 2190, $844 \mathrm{~cm}^{-1}$; MS (EI): $\mathrm{m} / \mathrm{z}(\%): 316(<1)\left[M^{+}\right], 301(<1)$; HRMS (EI): $m / z$ : calcd for $\mathrm{C} \mathrm{H}_{5} \mathrm{Q}_{5} \mathrm{Si}$ : $316.1706\left[M^{+}\right]$; found 316.1701; elemental analysis calcd (\%) for $\mathrm{C}_{15} \mathrm{H}_{28} \mathrm{O}_{5} \mathrm{Si}$ (316.46): $\mathrm{C} 56.93, \mathrm{H}$ 8.92; found: C 56.92 , H 8.95.

2,6-Anhydro-7,8-dideoxy-1,3,4,5-tetra-O-methyl-d-glycero-1-gulo-oct-7 ynitol (6): $\mathrm{NaOH}(1 \mathrm{n}, 3.9 \mathrm{~mL})$ was added to a solution of $5(652 \mathrm{mg}$ $2.06 \mathrm{mmol})$ in $\mathrm{CH}_{2} \mathrm{Cl}_{2} / \mathrm{MeOH}(5: 1,72 \mathrm{~mL})$. The mixture was stirred at room temperature for $2 \mathrm{~h}$, neutralized with a solution of $\mathrm{HCl} 1 \mathrm{n}$ and ex- tracted with EtOAc. The organic layer was washed with brine, driedover $\mathrm{Na}_{2} \mathrm{SO}_{\text {}}$ and concentrated under reduced pressure. The residue waspurified by silica gel column chromatography (hexanes/EtOAc 70:30) to give 6 ( $462 \mathrm{mg}, 1.89 \mathrm{mmol}, 92 \%)$ as an oil: [a] $]_{\mathrm{D}}=+132 \mathrm{~cm}^{3} \mathrm{~g}^{-1} \mathrm{dm}^{-1}(\mathrm{c}=1.64$ in $\left.\mathrm{CHCl}_{3}\right) ;{ }^{1} \mathrm{H} \mathrm{NMR}\left(500 \mathrm{MHz}, \mathrm{CDCl}_{3}\right): \mathrm{d}=2.58(\mathrm{~d}, J=9.3 \mathrm{~Hz}, 1 \mathrm{H}), 3.16$ (dd, $J=10.1,9.0 \mathrm{~Hz}, 1 \mathrm{H}$ ), 3.25 (dd, $J=9.5,5.9 \mathrm{~Hz}, 1 \mathrm{H}$ ), 3.39 (s, $3 \mathrm{H}), 3.47$ (dd, $J=9.3,9.2 \mathrm{~Hz}, 1 \mathrm{H}), 3.51(\mathrm{~s}, 3 \mathrm{H}), 3.54(\mathrm{~s}, 3 \mathrm{H}), 3.59$ (d, $J=3.4 \mathrm{~Hz}$, $2 \mathrm{H}$ ), $3.64(\mathrm{~s}, 3 \mathrm{H}$ ), 3.83 (ddd, $J=10.1,3.0,3.0 \mathrm{~Hz}, 1 \mathrm{H}$ ), $4.84 \mathrm{ppm}$ (dd, $J=$ 5.6, $2.3 \mathrm{~Hz}, 1 \mathrm{H}) ;{ }^{13} \mathrm{CNMR}\left(100.6 \mathrm{MHz}, \mathrm{CDCl}_{3}\right): \mathrm{d}=58.4\left(\mathrm{CH}_{3}\right), 58.8$ $\left(\mathrm{CH}_{3}\right), 60.1\left(\mathrm{CH}_{3}\right), 60.4\left(\mathrm{CH}_{3}\right), 65.6(\mathrm{CH}), 70.9(\mathrm{CH}), 73.1\left(\mathrm{CH}_{2}\right), 77.3$
(CH), $77.8(\mathrm{C}), 78.8(\mathrm{CH}), 80.4(\mathrm{CH}), 84.1 \mathrm{ppm}(\mathrm{CH})$; IR (film): $\tilde{n}=$ 3254, 2933, $2112 \mathrm{~cm}^{-1}$; MS (EI): $m / z(\%): 244(<1)\left[M^{+}\right], 199$ (7); HRMS (EI): $\mathrm{m} / \mathrm{z}$ calcd for $\mathrm{C}_{12} \mathrm{H}_{20} \mathrm{O}_{5}: 244.1311$ [ $M^{+}$]; found: 244.1306 ; el- emental analysis calcd (\%) for $\mathrm{C}_{12} \mathrm{H}_{20} \mathrm{O}_{5}$ (244.28): C 59.00, $\mathrm{H}$ 8.25; found: C 59.14, H 8.37 .

2,6-Anhydro-7,8-dideoxy-1,3,4,5-tetra-O-methyl-8-phenyl-d-glycero-1gulo-oct-7-ynitol (8): Compound $6(310 \mathrm{mg}, 1.27 \mathrm{mmol})$ in piperidine $(7$ $\mathrm{mL}$ ) was added to a suspension of $\left[\mathrm{Pd}\left(\mathrm{PPh}_{3}\right)_{4}\right](73 \mathrm{mg}, 0.06 \mathrm{mmol}), \mathrm{CuI}(24$ $\mathrm{mg}, 0.13 \mathrm{mmol})$, and $\mathrm{PhI}(140 \mathrm{~mL}, 1.27 \mathrm{mmol})$ in piperidine $(12 \mathrm{~mL})$ at room temperature under nitrogen. The mixture was stirred at $808 \mathrm{C}$ for $1 \mathrm{~h}$, diluted with EtOAc, washed with an aqueous saturated sol- ution of $\mathrm{NH}_{4} \mathrm{Cl}$, and the organic layer dried and concentrated under re- duced pressure. The residue was purified by silica gel column chromatog- raphy (hexanes/EtOAc 7:3) to give $8(359 \mathrm{mg}, 1.12 \mathrm{mmol}, 88 \%$ ) as a yel- lowish oil: $[\mathrm{a}]_{\mathrm{D}}=+183 \mathrm{~cm}^{3} \mathrm{~g}^{-1} \mathrm{dm}^{-1}\left(\mathrm{c}=2.15 \mathrm{in}^{\mathrm{n}} \mathrm{CHCl}_{3}\right) ;{ }^{1} \mathrm{H} \mathrm{NMR} \quad(500 \mathrm{MHz}$, $\left.\mathrm{CDCl}_{3}\right): \mathrm{d}=3.31(\mathrm{dd}, J=9.8,9.2 \mathrm{~Hz}, 1 \mathrm{H}), 3.33(\mathrm{dd}, J=9.5$,

$5.6 \mathrm{~Hz}, 1 \mathrm{H}$ ), 3.40 (s, 3H), 3.51 (s, 3H), 3.53 (dd, $J=9.3,9.3 \mathrm{~Hz}, 1 \mathrm{H}), 3.55$ (s, $3 \mathrm{H}), 3.606$ (d, $J=2.3 \mathrm{~Hz}, 1 \mathrm{H}$ ), 3.61 (d, $J=4.2 \mathrm{~Hz}, 1 \mathrm{H}), 3.65$ (s, $3 \mathrm{H}$ ), 3.91 (ddd, $J=9.8,3.9,2.3 \mathrm{~Hz}, 1 \mathrm{H}$ ), $5.06(\mathrm{~d}, J=5.6 \mathrm{~Hz}, 1 \mathrm{H}), 7.30(\mathrm{~m}, 3 \mathrm{H})$, $7.46 \mathrm{ppm}(\mathrm{m}, 2 \mathrm{H}) ;{ }^{13} \mathrm{C} \mathrm{NMR}\left(100.6 \mathrm{MHz}, \mathrm{CDCl}_{3}\right): \mathrm{d}=59.0\left(\mathrm{CH}_{3}\right), 59.6$ $\left(\mathrm{CH}_{3}\right), 60.8\left(\mathrm{CH}_{3}\right), 61.1\left(\mathrm{CH}_{3}\right), 67.0(\mathrm{CH}), 71.7\left(\mathrm{CH}_{2}\right), 73.8(\mathrm{CH}), 79.7$ $(\mathrm{CH}), 81.4(\mathrm{CH}), 83.9(\mathrm{C}), 84.9(\mathrm{CH}), 89.7(\mathrm{C}), 122.7(\mathrm{C}), 128.5(2 \times \mathrm{CH})$, $128.9(\mathrm{CH}), 132.4 \mathrm{ppm}(2 \times \mathrm{CH}) ; \mathrm{IR}(\mathrm{film}): \tilde{n}=2931,2226,1100 \mathrm{~cm}^{-1}$;MS (EI): $m / z(\%): 305(<1)\left[M^{+}-M e\right], 288(4)$; HRMS (EI): $m / z$ calcd for $\mathrm{C}_{17} \mathrm{H}_{21} \mathrm{O}_{5}$ : 305.1389 [ $M^{+}$-Me]; found: 305.1396; elemental analysis calcd (\%) for $\mathrm{C}_{18} \mathrm{H}_{24} \mathrm{O}_{5}$ (320.38):C 67.48, $\mathrm{H}$ 7.55; found: C 67.52, $\mathrm{H}$ 7.79. 3,7-Anhydro-4,5,6,8-tetra-O-methyl-1-phenyl-d-glycero-d-ido-octos-2ulose (9): Prepared from 8 ( $82 \mathrm{mg}, 0.25 \mathrm{mmol}$ ) following the general procedure (Method A) using dimethyl sulfide ( $40 \mathrm{~mL}, 0.54 \mathrm{mmol}$ ). The mixture was stirred at room temperature in the dark for $6 \mathrm{~h}$ and concentrat- ed under reduced pressure to give $9(106 \mathrm{mg})$ that was unstable on silica gel and alumina. Notwithstanding, the crude residue (yellow oil) was pure enough to allow spectroscopic characterization. ${ }^{1} \mathrm{H}$ N M R $(500 \mathrm{MHz}$, $\left.\mathrm{CDCl}_{3}\right): \mathrm{d}=2.86(\mathrm{~s}, 3 \mathrm{H}), 3.22(\mathrm{~s}, 3 \mathrm{H}), 3.34(\mathrm{~s}, 3 \mathrm{H}), 3.39-3.45(\mathrm{~m}, 2 \mathrm{H})$, 3.44 (s, $3 \mathrm{H}$ ), 3.50 (dd, $J=8.6,8.2 \mathrm{~Hz}, 1 \mathrm{H}$ ), $3.64(\mathrm{~m}, 2 \mathrm{H}$ ), 4.65 (ddd, $J=$ 9.7, 2.6, 2.6 Hz, $1 \mathrm{H}), 5.67(\mathrm{~d}, J=7.4 \mathrm{~Hz}, 1 \mathrm{H}), 6.96(\mathrm{~m}, 2 \mathrm{H}), 7.07(\mathrm{~m}, 1 \mathrm{H})$, $7.98 \mathrm{ppm}(\mathrm{m}, 2 \mathrm{H}) ;{ }^{13} \mathrm{C} \mathrm{NMR}\left(100.6 \mathrm{MHz}, \mathrm{CDCl}_{3}\right): \mathrm{d}=58.5\left(\mathrm{CH}_{3}\right), 59.0$ $\left(\mathrm{CH}_{3}\right), 59.9\left(\mathrm{CH}_{3}\right), 60.1\left(\mathrm{CH}_{3}\right), 71.8\left(\mathrm{CH}_{2}\right), 74.7(\mathrm{CH}), 75.5(\mathrm{CH}), 79.2$ $(\mathrm{CH}), 81.3(\mathrm{CH}), 84.2(\mathrm{CH}), 128.7(\mathrm{CH}), 130.3(2 \times \mathrm{CH}), 133.1(\mathrm{C}), 133.9$ $(2 \times \mathrm{CH}), 190.8(\mathrm{C}), 203.7 \mathrm{ppm}(\mathrm{C}) ;$ IR (film): $\tilde{n}=1716,1672,1450 \mathrm{~cm}^{-1}$, MS (EI): $m / z$ (\%): 352 (2) [ $\left.M^{+}\right], 247$ (4), 219 (18); HRMS (EI): $m / z$ calcd for $\mathrm{C}_{18} \mathrm{H}_{24} \mathrm{O}_{7}$ : $352.1522\left[M^{+}\right]$; found: 352.1527 .

Photolysis of 9: A deoxygenated solution of crude diketone $9(106 \mathrm{mg})$ in dry $\mathrm{C}_{6} \mathrm{D}_{6}(0.7 \mathrm{~mL})$ was placed in a resonance tube and irradiated with a daylight-lamp at $30 \mathrm{8C}$. The reaction was monitored by ${ }^{1} \mathrm{H}$ N MR spectroscopy, and complete consumption of starting material was observed after 9 $\mathrm{h}$, leading to exclusive formation of intermediate photoenol III. The solution could be concentrated at low temperature under reduced pres- sure but the photoenol was not stable enough to withstand chromato- graphic purification (silica gel or alumina). After silica gel column chromatography (hexanes/Et0Ac, 75:25) only the known lactone $10^{[45]}$ ( $28 \mathrm{mg}, 0.12 \mathrm{mmol}, 48 \%$ from 8 ) could be obtained.

4,8-Anhydro-1-deoxy-5,6,7,9-tetra-O-methyl-d-glycero-d-ido-nono-2,3diulose (1): Prepared from 7 (69 mg, $0.27 \mathrm{mmol}$ ) following the general procedure (Method A) using dimethyl sulfide ( $40 \mathrm{~mL}, 0.54 \mathrm{mmol})$. The mixture was stirred at room temperature in the dark for $6 \mathrm{~h}$ and concentrated under reduced pressure to give $1(80 \mathrm{mg})$, which was unstable on silica gel and alumina. Notwithstanding, the crude residue (yellow oil) was pure enough to allow spectroscopic characterization. ${ }^{1} \mathrm{H} \mathrm{NMR} \quad(400 \mathrm{MHz}$, $\left.\mathrm{CDCl}_{3}\right): \mathrm{d}=2.25(\mathrm{~s}, 3 \mathrm{H}), 3.16(\mathrm{dd}, J=9.8,7.7 \mathrm{~Hz}, 1 \mathrm{H}), 3.29$ (dd, $J=8.2,7.7 \mathrm{~Hz}, 1 \mathrm{H}), 3.35$ (s, $3 \mathrm{H}), 3.37(\mathrm{~s}, 3 \mathrm{H}), 3.49(\mathrm{~s}, 3 \mathrm{H}), 3.51(\mathrm{dd}$, $J=10.6,4.1 \mathrm{~Hz}, 1 \mathrm{H}), 3.55$ (s, $3 \mathrm{H}), 3.59$ (dd, $J=10.6,2.4 \mathrm{~Hz}, 1 \mathrm{H}), 3.64$ (dd, $J=7.9,7.4 \mathrm{~Hz}, 1 \mathrm{H}$ ), 4.18 (ddd, $J=9.8,4.2,2.1 \mathrm{~Hz}, 1 \mathrm{H}$ ), $5.48 \mathrm{ppm}$ (d, $J=7.4 \mathrm{~Hz}, 1 \mathrm{H}) ;{ }^{13} \mathrm{C} \mathrm{NMR}\left(100.6 \mathrm{MHz}, \mathrm{CDCl}_{3}\right): \mathrm{d}=23.3\left(\mathrm{CH}_{3}\right), 59.1$ $\left(\mathrm{CH}_{3}\right), 59.2\left(\mathrm{CH}_{3}\right), 59.8\left(\mathrm{CH}_{3}\right), 60.1\left(\mathrm{CH}_{3}\right), 71.5\left(\mathrm{CH}_{2}\right), 72.3(\mathrm{CH}), 74.1$ $(\mathrm{CH}), 78.5(\mathrm{CH}), 80.6(\mathrm{CH}), 82.7(\mathrm{CH}), 197.1(\mathrm{C}), 200.3 \mathrm{ppm}(\mathrm{C})$; IR (film): $\mathrm{n}^{=}=2934,2933,1715 \mathrm{~cm}^{-1}$; MS (EI): $m / z(\%): 290(<1)\left[M^{+}\right], 247$ 
(2); HRMS (EI): $m / z$ calcd for $\mathrm{C}_{13} \mathrm{H}_{22} \mathrm{O}_{7}: 290.1366\left[M^{+}\right]$; found: 290.1363 . Photocyclization of 1 : A solution of crude diketone $1(80 \mathrm{mg})$ in dry

$\mathrm{C}_{6} \mathrm{~B}(1 \mathrm{~mL})$, was placed in a resonance tube and irradiated with a daylight lamp at $308 \mathrm{C}$ for $3 \mathrm{~h}$. The mixture was concentrated under reduced pressure and the residue purified by silica gel column chromatography (hexanes/EtOAc 7:3), to give $(2 S, 3 \mathrm{a} S, 4 S, 5 R, 6 S, 6 \mathrm{a} R)$-2-hydroxy-4,5,6-trimethoxy-6a-(methoxymethyl)-2-methyltetrahydro- $2 H$-cyclopenta[b]furan-3(3aH)-one (2) ( $41 \mathrm{mg}, 0.14 \mathrm{mmol}, 52 \%$ from 7 ) as a crystalline solid. M.p. 96.6-97.2 8C ( $n$-hexane/EtOAc); $[\mathrm{a}]_{\mathrm{D}}=+138 \mathrm{~cm}^{3} \mathrm{~g}^{-1} \mathrm{dm}^{-1}(c=1.22$ in $\left.\mathrm{CHCl}_{3}\right) ;{ }^{1} \mathrm{H} \mathrm{NMR}\left(500 \mathrm{MHz}, \mathrm{C} \mathrm{D}_{6} 6\right.$ note: the hydrogen atoms at $\mathrm{C} 5$ and $\mathrm{C} 6$ have very similar chemical shifts and as a consequence the cou- pled hydrogen at C4 appears as a complex signal by virtual coupling. The data of hydrogens at $\mathrm{C} 4-\mathrm{C} 6$ shown below have been calculated by itera- tive simulation using program DAISY as implemented in Bruker Topspin v. 2.1): d = 1.64 (d, $J=0.9 \mathrm{~Hz}, 3 \mathrm{H}), 2.73(\mathrm{~s}, 3 \mathrm{H}), 3.09$ (d, $J=4.7 \mathrm{~Hz}, 1 \mathrm{H})$, $3.17(\mathrm{~s}, 3 \mathrm{H}), 3.25$ (s, 6H), 3.30 (d, $J=10.6 \mathrm{~Hz}, 1 \mathrm{H}), 3.49$ (d, $J=10.3 \mathrm{~Hz}$, $1 \mathrm{H}$ ), 3.64 (complex signal, d, $J=8.2 \mathrm{~Hz}, 1 \mathrm{H}$ ), 3.65 (complex signal, dd, $J=8.2,6.3 \mathrm{~Hz}, 1 \mathrm{H}$ ), 3.78 (complex signal, dd, $J=6.3,4.7 \mathrm{~Hz}, 1 \mathrm{H}$ ), 5.21 $\operatorname{ppm}(\mathrm{d}, J=0.6 \mathrm{~Hz}, 1 \mathrm{H}, \mathrm{OH}) ;{ }^{1} \mathrm{H} \mathrm{NMR}\left(500 \mathrm{MHz}, \mathrm{CDCl}_{3}\right): \mathrm{d}=1.41$ (d, $J$ $=0.9 \mathrm{~Hz}, 3 \mathrm{H}), 3.08(\mathrm{~d}, J=4.1 \mathrm{~Hz}, 1 \mathrm{H}), 3.42(\mathrm{~s}, 3 \mathrm{H}), 3.46(\mathrm{~s}, 3 \mathrm{H})$, $3.48(\mathrm{~s}, 3 \mathrm{H}), 3.50(\mathrm{~s}, 3 \mathrm{H}), 3.58(\mathrm{~d}, J=10.4 \mathrm{~Hz}, 1 \mathrm{H}), 3.63(\mathrm{dd}, J=6.1$ $1.2 \mathrm{~Hz}, 1 \mathrm{H}$ ), 3.68 (dd, $J=6.0,5.0 \mathrm{~Hz}, 1 \mathrm{H}$ ), 3.70 (ddd, $J=5.0,4.2,0.9 \mathrm{~Hz}$, $1 \mathrm{H}), 3.71(\mathrm{~d}, J=10.4 \mathrm{~Hz}, 1 \mathrm{H}), 5.05 \mathrm{ppm}(\mathrm{brd}, J=0.9 \mathrm{~Hz}, 1 \mathrm{H}, 0 \mathrm{H}$ ); ${ }^{13} \mathrm{C} \mathrm{N}$ MR $\left(100.6 \mathrm{MHz}, \mathrm{C}_{6} \mathrm{D}_{6}\right): \mathrm{d}=21.1\left(\mathrm{CH}_{3}\right), 51.2(\mathrm{CH}), 57.5\left(\mathrm{CH}_{3}\right), 58.1$ $\left(\mathrm{CH}_{3}\right), 58.7\left(\mathrm{CH}_{3}\right), 59.0\left(\mathrm{CH}_{3}\right), 74.2\left(\mathrm{CH}_{2}\right), 84.2(\mathrm{CH}), 87.6(\mathrm{C}), 88.4$ $(\mathrm{CH}), 90.8(\mathrm{CH}), 99.7(\mathrm{C}), 208.1 \mathrm{ppm}(\mathrm{C})$; IR (film): $\mathrm{n}^{\sim}=3337,2949,2836$, $1760 \mathrm{~cm}^{-1}$; MS (FAB): $m / z(\%): 290$ (2) $\left[M^{+}\right], 273$ (100); HR MS (FAB): $\mathrm{m} / \mathrm{z}$ : calcd for $\mathrm{C}_{13} \mathrm{H}_{22} \mathrm{O}_{7}: 290.1366\left[M^{+}\right]$; found: 290.1372 ; elemental analysis calcd (\%) for $\mathrm{C}_{13} \mathrm{H}_{22} \mathrm{O}_{7}$ (290.31): $\mathrm{C}$ 53.78, $\mathrm{H}$ 7.64; found: $\mathrm{C} 53.71 ; \mathrm{H}$ 7.73. Crystal data and structure refinement for compound 2: $\mathrm{C}_{13} \mathrm{H}_{22} \mathrm{O}_{7}, M_{t}$ $=290.31$, orthorhombic, space group $P 2_{1} 2_{1} 2_{1}, a=7.9261(6) \AA, b=$ 9.2758(8) $\AA, c=19.6176(17) \AA, \quad b=908, V=1442.3(2) \AA^{3}, Z=4,1_{\text {calcd }}=$ $1.337 \mathrm{mg} \mathrm{m}^{-3}, \mathrm{~m}\left(\mathrm{Mo}_{\mathrm{Ka}}\right)=0.71073 \AA, F(000)=624, T=123(2) \mathrm{K}$, colorless crystal, $0.45 \times 0.20 \times 0.12 \mathrm{~mm}^{3}$, collected reflections 12479 . The structure was solved by direct method, all hydrogen atoms were refined anisotropically using full-matrix least-squared based $F_{2}$ to give $R_{1}=0.0415, w R_{2}=$ 0.0689 for $3028 \mathrm{independently} \mathrm{observed} \mathrm{reflections}\left(\mathrm{j} F_{\mathrm{o}} \mathrm{j}>2 \mathrm{~s}\left(\mathrm{j} F_{\mathrm{o}} \mathrm{j}\right)\right)$ and 191 parameters.

\section{Irradiation with pyrene: A solution of crude diketone 1 (14 mg,}

$0.048 \mathrm{mmol}$ ) and pyrene (126 mg, $0.624 \mathrm{mmol})$ in dry $\mathrm{C}_{6} \mathrm{D}_{6}(1.2 \mathrm{~mL})$, was placed in a resonance tube and irradiated with a daylight lamp at $30 \mathrm{8C}$ for $12 \mathrm{~h}$. No reaction was detected by using ${ }^{1} \mathrm{H}$ N MR spectroscopy under these conditions.

4,8-Anhydro-5,6,7,9-tetra-O-benzyl-1-deoxy-d-glycero-d-ido-nono-2,3diulose (18): Prepared from 11 (70 mg, $0.125 \mathrm{mmol}$ ) following the general procedure (Method B) using $\mathrm{NaIO}_{4}(107 \mathrm{mg}, 0.5 \mathrm{mmol})$ and $\mathrm{RuO}_{2} \cdot x \mathrm{H}_{2} \mathrm{O}$ $(1.1 \mathrm{mg})$. The reaction mixture was stirred at room temperature in the dark for $1 \mathrm{~h}$. The residue was purified by rapid silica gel column chromatography (hexanes/EtOAc 9:1) to give $18(46 \mathrm{mg}, 0.077 \mathrm{mmol}, 62 \%$ ) as a yellow oil: $[\mathrm{a}]_{\mathrm{D}}=+52 \mathrm{~cm}^{3} \mathrm{~g}^{-1} \mathrm{dm}^{-1}\left(\mathrm{c}=0.65 \mathrm{in}_{\mathrm{CHCl}}\right) ;{ }^{1} \mathrm{H} \mathrm{N} \mathrm{MR}$ (500 $\mathrm{MHz}_{\mathrm{CDCl}}$ ): $\mathrm{d}=2.07(\mathrm{~s}, 3 \mathrm{H}), 3.61(\mathrm{dd}, J=9.8,8.0 \mathrm{~Hz}, 1 \mathrm{H}), 3.64$ (dd, $J=10.9,3.6 \mathrm{~Hz}, 1 \mathrm{H}$ ), 3.67 (dd, $J=10.9,2.4 \mathrm{~Hz}, 1 \mathrm{H}$ ), 3.73 (dd, $J=8.3$, $8.0 \mathrm{~Hz}, 1 \mathrm{H}$ ), 3.99 (dd, $J=8.3,7.1 \mathrm{~Hz}, 1 \mathrm{H}$ ), 4.37 (ddd, $J=9.8,3.6,2.4 \mathrm{~Hz}$, $1 \mathrm{H}), 4.45$ (d, $J=10.9 \mathrm{~Hz}, 1 \mathrm{H}), 4.46(\mathrm{~d}, J=12.1 \mathrm{~Hz}, 1 \mathrm{H}), 4.49(\mathrm{~d}, J=$ $10.9 \mathrm{~Hz}, 1 \mathrm{H}$ ), 4.57 (d, $J=11.6 \mathrm{~Hz}, 2 \mathrm{H}$ ), 4.69 (d, $J=10.9 \mathrm{~Hz}, 1 \mathrm{H}$ ), 4.75 (s, $2 \mathrm{H}), \quad 5.56(\mathrm{~d}, J=7.4 \mathrm{~Hz}, 1 \mathrm{H}), 7.09-7.30 \mathrm{ppm}(\mathrm{m}, 20 \mathrm{H}) ;{ }^{13} \mathrm{C} \mathrm{NMR}$ $\left(100.6 \mathrm{MHz}, \mathrm{CDCl}_{3}\right): \mathrm{d}=23.5\left(\mathrm{CH}_{3}\right), 68.9\left(\mathrm{CH}_{2}\right), 72.4(\mathrm{CH}), 73.4\left(\mathrm{CH}_{2}\right)$, $74.0\left(\mathrm{CH}_{2}\right), 74.5\left(\mathrm{CH}_{2}\right), 74.6(\mathrm{CH}), 74.9\left(\mathrm{CH}_{2}\right), 77.1(\mathrm{CH}), 78.5(\mathrm{CH}), 81.2$ $(\mathrm{CH}), 127.6-128.4(20 \times \mathrm{CH}), 136.8(\mathrm{C}), 137.9(\mathrm{C}), 138.1(\mathrm{C}), 138.2(\mathrm{C})$ 197.4 (C), $200.7 \mathrm{ppm}(\mathrm{C})$; IR (film): $\tilde{\mathrm{n}}=2928,1721 \mathrm{~cm}^{-1}$; MS (EI): $\mathrm{m} / \mathrm{z}(\%)$ $594(<1)\left[M^{+}\right], 503$ (2); HRMS (EI): $m / z$ calcd for $\mathrm{C}_{37} \mathrm{H}_{38} \mathrm{O}_{7}$ : 594.2618 $\left[M^{+}\right]$; found: 594.2622; elemental analysis calcd (\%) for $\mathrm{C}_{37} \mathrm{H}_{38} \mathrm{O}_{7}$ (594.69): C 74.73, H 6.44; found: C 74.80, H 6.29.

Photocyclization of 18: A deoxygenated solution of diketone 18 (30 mg, $0.05 \mathrm{mmol})$ in purified $\mathrm{CDCl}_{3}(0.6 \mathrm{~mL})$, was placed in a resonance tube and irradiated with a daylight lamp at $30 \mathrm{8C}$ for $2 \mathrm{~h}$. The mixture was concentrated under reduced pressure and the residue was purified by silica gel column chromatography (hexanes/EtOAc 9:1), to give $(2 S, 3 \mathrm{a} S, 4 S, 5 R, 6 S, 6 \mathrm{a} R)-4,5,6$-tris(benzyloxy)-6a-[(benzyloxy)methyl]-2-hydroxy-2-methyltetrahydro- $2 H$-cyclopenta $[b]$ furan-3(3a $H)$-one $(17 \mathrm{mg}, 0.029 \mathrm{mmol}, 58 \%)$ as a colorless oil: $[\mathrm{a}]_{\mathrm{D}}=+31 \mathrm{~cm}^{3} \mathrm{~g}^{-1} \mathrm{dm}^{-1}(\mathrm{c}=$ $0.9 \mathrm{in} \mathrm{CHCl})_{3}{ }^{1} \mathrm{H} \mathrm{NMR}(500 \mathrm{MHz}, \mathrm{CDCl}): \mathrm{g}=1.37(\mathrm{~s}, 3 \mathrm{H}), 3.21(\mathrm{~m}$, $1 \mathrm{H}), 3.77(\mathrm{~d}, J=10.6 \mathrm{~Hz}, 1 \mathrm{H}), 3.89$ (d, $J=10.6 \mathrm{~Hz}, 1 \mathrm{H}), 3.94(\mathrm{~m}, 1 \mathrm{H})$, $4.00(\mathrm{~m}, 2 \mathrm{H}), 4.57-4.76(\mathrm{~m}, 8 \mathrm{H}), 5.09(\mathrm{~s}, 1 \mathrm{H}), 7.23-7.39 \mathrm{ppm}(\mathrm{m}, 20 \mathrm{H})$; ${ }^{13} \mathrm{CNMR}\left(100.6 \mathrm{MHz}, \mathrm{CDCl}_{3}\right): \mathrm{d}=20.6\left(\mathrm{CH}_{3}\right), 51.6(\mathrm{CH}), 71.8\left(\mathrm{CH}_{2}\right)$, $72.0\left(\mathrm{CH}_{2}\right), 72.6\left(\mathrm{CH}_{2}\right), 73.3\left(\mathrm{CH}_{2}\right), 74.3\left(\mathrm{CH}_{2}\right), 82.6(\mathrm{CH}), 85.0(\mathrm{CH})$, $87.7(\mathrm{C}), 88.5(\mathrm{CH}), 99.2(\mathrm{C}), 127.7-128.8(20 \times \mathrm{CH}), 135.9(\mathrm{C}), 137.5(\mathrm{C})$, 137.6 (C), 137.8 (C), $208.3 \mathrm{ppm}$ (C); IR (film): $\tilde{n=} 3370,2918,1762 \mathrm{~cm}^{-1}$; MS (EI): $m / z(\%): 594(<1)\left[M^{+}\right], 576(<1)$; HRMS (EI): $m / z$ calcd for $\mathrm{C}_{37} \mathrm{H}_{38} \mathrm{O}_{7}: 594.2618\left[M^{+}\right]$; found: 594.2615; elemental analysis calcd (\%) for $\mathrm{C}_{37} \mathrm{H}_{38} \mathrm{O}_{7}$ (594.69): C 74.73, H 6.44; found: C 74.37, $\mathrm{H} 6.83$.

4,8-Anhydro-5,6,7-tri-O-benzyl-9-O-(tert-butyldiphenylsilyl)-1-deoxy-dglycero-d-ido-nono-2,3-diulose (19): Prepared from 12 (30 mg, $0.042 \mathrm{mmol}$ ) following the general procedure (Method B) using $\mathrm{NaIO}_{4}$ (36 $\mathrm{mg}, 0.168 \mathrm{mmol})$ and $\mathrm{RuO}_{2} \cdot x \mathrm{H}_{2} \mathrm{O}(0.5 \mathrm{mg})$. The reaction mixture was stirred at room temperature in the dark for $1 \mathrm{~h}$. The residue was purified by rapid silica gel column chromatography (hexanes/EtOAc 97:3) to give 19 (20 mg, $0.027 \mathrm{mmol}, 64 \%)$ as a yellow oil: $[\mathrm{a}]_{\mathrm{D}}=+36 \mathrm{~cm}^{3} \mathrm{~g}^{-1} \mathrm{dm}^{-1}(c=$ 1.87 in $\left.\mathrm{CHCl}_{3}\right)$; ${ }^{1} \mathrm{H}$ NMR (400 MHz, $\left.\mathrm{CDCl}_{3}\right): \mathrm{d}=1.07(\mathrm{~s}, 9 \mathrm{H}), 2.09(\mathrm{~s}, 3 \mathrm{H})$, $3.81(\mathrm{~m}, 2 \mathrm{H}), 3.92(\mathrm{dd}, J=11.7,3.2 \mathrm{~Hz}, 1 \mathrm{H}), 3.95(\mathrm{dd}, J=11.7,2.1 \mathrm{~Hz}, 1$ H), 4.01 (dd, $J=8.5,7.2 \mathrm{~Hz}, 1 \mathrm{H}), 4.31(\mathrm{~m}, 1 \mathrm{H}), 4.58(\mathrm{~d}, J=10.9 \mathrm{~Hz}, 1 \mathrm{H})$, $4.66(\mathrm{~d}, J=10.4 \mathrm{~Hz}, 2 \mathrm{H}), 4.82(\mathrm{~s}, 2 \mathrm{H}), 4.84(\mathrm{~d}, J=11.1 \mathrm{~Hz}, 1 \mathrm{H}), 5.62(\mathrm{~d}, J$ $=7.4 \mathrm{~Hz}, 1 \mathrm{H}), 7.16-7.73 \mathrm{ppm}(\mathrm{m}, 25 \mathrm{H}) ;{ }^{13} \mathrm{C} \mathrm{NMR}\left(100.6 \mathrm{MHz}, \mathrm{CDCl}_{3}\right)$ : $\mathrm{d}=19.3(\mathrm{C}), 23.6\left(\mathrm{CH}_{3}\right), 26.5\left(3 \times \mathrm{CH}_{3}\right), 62.9\left(\mathrm{CH}_{2}\right)$, $72.1(\mathrm{CH}), 74.2(\mathrm{CH}), 74.7(\mathrm{CH})_{2} 75.2(\mathrm{CH})_{12} 75.9(\mathrm{CH}), 76.9(\mathrm{CH}), 79.1$ $(\mathrm{CH}), 81.6(\mathrm{CH}), 127.6-135.8(25 \times \mathrm{CH}), 133.2-138.2(5 \times \mathrm{C}), 197.6(\mathrm{C})$, 200.7 ppm (C); IR (film): $\tilde{n}=2928,1713 \mathrm{~cm}^{-1}$; MS (EI): $m / z(\%): 742(<1)$ $\left[M^{+}\right], 685(<1)$; HRMS (EI): $m / z$ calcd for $\mathrm{C}_{46} \mathrm{H}_{50} \mathrm{O}_{7} \mathrm{Si}: 742.3326$ $\left[M^{+}\right]$; found: 742.3345; elemental analysis calcd (\%) for $\mathrm{C}_{46} \mathrm{H}_{50} \mathrm{O}_{7} \mathrm{Si}$ (742.97): C 74.36, H 6.78; found: C 74.52, H 6.98.

Photocyclization of 19: A deoxygenated solution of diketone 19 (40 mg, $0.054 \mathrm{mmol})$ in purified C C Cl $(31.4 \mathrm{~mL})$, was placed in a resonance tube and irradiated with a daylight lamp at room temperature for $3 \mathrm{~h}$. The mixture was concentrated under reduced pressure and the residue puri- fied by silica gel column chromatography (hexanes/EtOAc 9:1) to give (3aS,4S,5R,6S,6aR)-4,5,6-tris(benzyloxy)-6a-[(tert-butyldiphenylsilyl)oxy]methyl-2-hydroxy-2-methyltetrahydro- $2 H$-cyclopenta[b]furan-3(3a $H)$-one (26) as a mixture of isomers (26 $\mathrm{mg}, 0.035 \mathrm{mmol}$, d.r. $5: 1,65 \%)$ as a colorless oil. ${ }^{1} \mathrm{H} \mathrm{NMR}\left(500 \mathrm{MHz}, \mathrm{CDCl}_{3}\right.$; complex spectrum, only clearly distinguished signals are reported): $d=$ major isomer $1.08(\mathrm{~s}, 9 \mathrm{H}), 1.44(\mathrm{~s}, 3$ $\mathrm{H}), 3.29(\mathrm{~d}, J=4.5 \mathrm{~Hz}, 1 \mathrm{H}), 5.09 \mathrm{ppm}(\mathrm{s}, 1 \mathrm{H})$; minor isomer $1.02(\mathrm{~s}$, $9 \mathrm{H}), 1.49(\mathrm{~s}, 3 \mathrm{H}), 3.31 \mathrm{ppm}(\mathrm{d}, J=3.7 \mathrm{~Hz}, 1 \mathrm{H}) ;{ }^{13} \mathrm{C}$ N MR $(100.6 \mathrm{MHz}$, $\mathrm{CDCl}_{3}$ ) (complex spectrum, only clearly distinguished signals are reported): $d$ (major isomer) $=19.1(\mathrm{C}), 20.3\left(\mathrm{CH}_{3}\right), 26.8\left(3 \times \mathrm{CH}_{3}\right), 50.9(\mathrm{CH})$, $66.0\left(\mathrm{CH}_{2}\right), 88.8(\mathrm{C}), 99.1(\mathrm{C}), 207.8 \mathrm{ppm}(\mathrm{C})$; d (minor isomer) $=19.2$ (C), $22.6\left(\mathrm{CH}_{3}\right), 26.8\left(3 \times \mathrm{CH}_{3}\right), 53.4(\mathrm{CH}), 65.1\left(\mathrm{CH}_{2}\right), 88.8(\mathrm{C}), 100.1$ (C), $210.7 \mathrm{ppm}$ (C); IR (film): $\tilde{\mathrm{n}}=3374,2928,1765 \mathrm{~cm}^{-1}$; MS $\left(\mathrm{ESI}^{+}\right.$): $\mathrm{m} / \mathrm{z}$ (\%): 765 (100) $\left[\mathrm{M}^{+}+\mathrm{Na}\right] ;$ HRMS $\left(\mathrm{ESI}^{+}\right): \mathrm{m} / \mathrm{z}$ calcd for $\mathrm{C}_{46} \mathrm{H}_{50} \mathrm{NaO}_{7} \mathrm{Si}$ : $765.3224\left[M^{+}+\mathrm{Na}\right]$; found: 765.3226 ; elemental analysis calcd (\%) for $\mathrm{C}_{46} \mathrm{H}_{50} \mathrm{O}_{7} \mathrm{Si}$ (742.97): $\mathrm{C}$ 74.36, H 6.78; found: $\mathrm{C} 74.66, \mathrm{H} \quad 6.57$. 9-O-Acetyl-4,8-anhydro-5,6,7-tri-O-benzyl-1-deoxy-d-glycero-d-ido-nono2,3-diulose (20): Prepared from 13 (70 $\mathrm{mg}, 0.136 \mathrm{mmol}$ ) following the

general procedure (Method B) using NaIO (116 mg, $0.542 \mathrm{mmol}$ ) and $\mathrm{RuO}_{2} \times \mathrm{H}_{2} \mathrm{O}(1.2 \mathrm{mg})$. The reaction mixture was stirred at room temperature in the dark for $45 \mathrm{~min}$. The residue was purified by rapid silica gel column chromatography (hexanes/EtOAc 9:1) to give 20 (35 mg, $0.064 \mathrm{mmol}, 47 \%)$ as a yellow oil: [a] $=\hbar_{\mathrm{D}} 89 \mathrm{~cm}^{3} \mathrm{~g}^{-1} \mathrm{dm}^{-1}(c=0.62 \mathrm{in}$ $\left.\mathrm{CHCl}_{3}\right)$; ${ }^{1} \mathrm{H} \mathrm{NMR}(500 \mathrm{MHz}, \mathrm{CDCl}): \mathrm{d}=2.05(\mathrm{~s}, 3 \mathrm{H}), 2.12(\mathrm{~s}, 3 \mathrm{H}), 3.49$ (dd, $J=9.8,7.2 \mathrm{~Hz}, 1 \mathrm{H}$ ), 3.80 (dd, $J=7.9,7.2 \mathrm{~Hz}, 1 \mathrm{H}$ ), 4.07 (dd, $J=7.8$, $7.2 \mathrm{~Hz}, 1 \mathrm{H}$ ), 4.21 (dd, $J=12.2,4.8 \mathrm{~Hz}, 1 \mathrm{H}), 4.33$ (dd, $J=12.2,2.1 \mathrm{~Hz}$, $1 \mathrm{H}$ ), 4.45 (ddd, $J=9.8,4.8,2.1 \mathrm{~Hz}, 1 \mathrm{H}), 4.503$ (d, $J=10.6 \mathrm{~Hz}, 1 \mathrm{H}), 4.506$ (d, $J=11.1 \mathrm{~Hz}, 1 \mathrm{H}), 4.59$ (d, $J=10.6 \mathrm{~Hz}, 1 \mathrm{H}), 4.74(\mathrm{~d}, J=10.9 \mathrm{~Hz}, 1 \mathrm{H})$, $4.76(\mathrm{~d}, J=11.4 \mathrm{~Hz}, 1 \mathrm{H}), 4.81$ (d, $J=11.3 \mathrm{~Hz}, 1 \mathrm{H}), 5.57$ (d, $J=7.2 \mathrm{~Hz}$, 1H), $7.10-7.35 \mathrm{ppm}(\mathrm{m}, 15 \mathrm{H}) ;{ }^{13} \mathrm{C} \mathrm{NMR}\left(100.6 \mathrm{MHz} \mathrm{CDCl}_{3}\right): \mathrm{d}=20.8$ 
$\left(\mathrm{CH}_{3}\right), 23.5\left(\mathrm{CH}_{3}\right), 63.4\left(\mathrm{CH}_{2}\right), 72.6(\mathrm{CH}), 72.9(\mathrm{CH}), 73.9\left(\mathrm{CH}_{2}\right), 74.1$ $\left(\mathrm{CH}_{2}\right), 74.6\left(\mathrm{CH}_{2}\right), 76.8(\mathrm{CH}), 78.1(\mathrm{CH}), 80.2(\mathrm{CH}), 127.8-128.5(15 \times$ CH), 136.7 (C), 137.6 (C), 137.9 (C), 170.8 (C), 197.2 (C), 199.6 ppm (C); IR (film): $\mathrm{n}^{2}=2928,1735 \mathrm{~cm}^{-1}$; MS (EI): $m / z$ (\%): 546 (2) [ $\left.M^{+}\right], 475$ (1); HRMS (EI): $m / z$ calcd for $\mathrm{C}_{32} \mathrm{H}_{34} \mathrm{O}_{8}: 546.2254$ [ $\left.M^{+}\right]$; found: 546.2236; elemental analysis calcd (\%) for $\mathrm{C}_{32} \mathrm{H}_{34} \mathrm{O}_{8}$ (546.61): C 70.31, $\mathrm{H}$ 6.27; found: C 70.34,H 6.27.

Photocyclization of 20: A deoxygenated solution of diketone $20(35 \mathrm{mg}$, $0.064 \mathrm{mmol})$ in purified $\mathrm{CDCl}_{3}(1 \mathrm{~mL})$, was placed in a resonance tube and irradiated with a daylight lamp at $308 \mathrm{C}$ for $1 \mathrm{~h}$. The mixture was concentrated under reduced pressure and the residue purified by silica gel column chromatography (hexanes/EtOAc 8:2) to give (3aS,4S,5R,6- $S, 6 \mathrm{a} R$ )4,5,6-tris(benzyloxy)-6a-(acetyloxy)methyl-2-hydroxy-2-methyltetrahydro- $2 H$-cyclopenta[b] furan-3(3aH)-one (27) a colorless oil as a mixture of isomers $(23.5 \mathrm{mg}, 0.043 \mathrm{mmol}$, d.r., 1:0.9, $67 \%)$ : ${ }^{1} \mathrm{H} \mathrm{NMR}$ $(500 \mathrm{M} \mathrm{Hz}, \mathrm{CDCl} \dot{\mathrm{B}}$ complex spectrum, only clearly distinguished signals are reported): $d=1.35(\mathrm{~s}, 3 \mathrm{H}), 1.42(\mathrm{~s}, 3 \mathrm{H}), 2.03(\mathrm{~s}, 3 \mathrm{H}), 2.09(\mathrm{~s}, 3 \mathrm{H})$, $3.07(\mathrm{~d}, J=1.9 \mathrm{~Hz}, 1 \mathrm{H}), 3.11$ (d, $J=3.2 \mathrm{~Hz}, 1 \mathrm{H}), 3.92 \mathrm{ppm}(\mathrm{d}, J=5.4 \mathrm{~Hz}$ $1 \mathrm{H}) ;{ }^{13} \mathrm{C}$ N MR $\left(100.6 \mathrm{M} \mathrm{Hz}, \mathrm{C}_{6} \mathrm{D}_{6}\right.$; complex spectrum, only clearly distinguished signals are reported): $d=20.8\left(\mathrm{CH}_{3}\right), 20.9\left(\mathrm{CH}_{3}\right), 21.5\left(\mathrm{CH}_{3}\right), 22.5$ $\left(\mathrm{CH}_{3}\right), 52.7(\mathrm{CH}), 54.9(\mathrm{CH}), 65.8\left(\mathrm{CH}_{2}\right), 65.9\left(\mathrm{CH}_{2}\right), 87.7(\mathrm{CH}), 87.9(\mathrm{C})$, 88.7 (C), 99.3 (C), 100.0 (C), 170.3 (C), 170.4 (C), 206.6 (C), 210.3 ppm (C); IR (film): $\tilde{n}^{2}=3424,2927,1742 \mathrm{~cm}^{-1} ; \mathrm{MS}$ (EI): $\mathrm{m} / z(\%): 546(<1)\left[M^{+}\right.$ ], 503 (<1); HRMS (EI): $m / z$ calcd for $\mathrm{C}_{32} \mathrm{H}_{34} \mathrm{O}_{8}: 546.2254\left[M^{+}\right]$; found: 546.2273 ; elemental analysis calcd (\%) for $\mathrm{C}_{32} \mathrm{H}_{34} \mathrm{O}_{8}$ (546.61): C 70.31, H 6.27; found: C 70.10, H 6.40 .

4,8-Anhydro-9-azido-5,6,7-tri-O-benzyl-1,9-dideoxy-d-glycero-d-idonono-2,3-diulose (21): Prepared from $14(60 \mathrm{mg}, 0.121 \mathrm{mmol})$ following the general procedure (Method B) using $\mathrm{NaIO}_{4}(103 \mathrm{mg}, 0.481 \mathrm{mmol})$ and $\mathrm{RuO}_{2} \times \mathrm{H}_{2} \mathrm{O}(1.1 \mathrm{mg})$. The reaction mixture was stirred at room temperature in the dark for $30 \mathrm{~min}$. The residue was purified by rapid silica gel column chromatography (hexanes/EtOAc 1:1) to give 21 (38 mg, $0.072 \mathrm{mmol}, 59 \%)$ as a yellow oil. $[\mathrm{a}]_{\mathrm{D}}=+52 \mathrm{~cm}^{3} \mathrm{~g}^{-1} \mathrm{dm}^{-1}(\mathrm{c}=2.32 \mathrm{in}$ $\left.\mathrm{CHCl}_{3}\right) ;{ }^{1} \mathrm{H} \mathrm{NMR}\left(400 \mathrm{MHz}, \mathrm{CDCl}_{3}\right): \mathrm{d}=2.12(\mathrm{~s}, 3 \mathrm{H}), 3.34(\mathrm{dd}, J=13.3$, $5.0 \mathrm{~Hz}, 1 \mathrm{H}), 3.51(\mathrm{dd}, J=9.8,7.2 \mathrm{~Hz}, 1 \mathrm{H}), 3.55(\mathrm{dd}, J=13.3,2.4 \mathrm{~Hz}, 1 \mathrm{H})$, 3.77 (dd, $J=8.0,7.4 \mathrm{~Hz}, 1 \mathrm{H}$ ), $4.08(\mathrm{dd}, J=8.2,7.4 \mathrm{~Hz}, 1 \mathrm{H}$ ), 4.43 (ddd, $J=$ 9.7, 4.6, $2.1 \mathrm{~Hz}, 1 \mathrm{H}), 4.50-4.82(\mathrm{~m}, 6 \mathrm{H}), 5.60(\mathrm{~d}, J=7.2 \mathrm{~Hz}, 1 \mathrm{H}), 7.18-$ $7.37 \mathrm{ppm}(\mathrm{m}, 15 \mathrm{H}) ;{ }^{13} \mathrm{C} \mathrm{NMR}\left(100.6 \mathrm{MHz}, \mathrm{CDCl}_{3}\right): \mathrm{d}=23.5\left(\mathrm{CH}_{3}\right), 51.7$ $\left(\mathrm{CH}_{2}\right), 72.6(\mathrm{CH}), 74.0(\mathrm{CH}), 74.1\left(\mathrm{CH}_{2}\right), 74.3\left(\mathrm{CH}_{2}\right), 74.7\left(\mathrm{CH}_{2}\right), 77.7$ $(\mathrm{CH}), 78.2(\mathrm{CH}), 80.2(\mathrm{CH}), 127.8-128.6(15 \times \mathrm{CH}), 136.7(\mathrm{C}), 137.7(\mathrm{C})$, 137.9 (C), 197.2 (C), $199.8 \mathrm{ppm}$ (C); IR (film): $\tilde{n}=2925,2099,1731$, $1715 \mathrm{~cm}^{-1}$; MS (ESI $\left.{ }^{+}\right): m / z(\%): 584(100)\left[M^{+}+\mathrm{MeOH}+\mathrm{Na}\right] ; \mathrm{HRMS}$ $\left(\mathrm{ESI}^{+}\right): \mathrm{m} / \mathrm{z}$ calcd for $\mathrm{C}_{31} \mathrm{H}_{35} \mathrm{~N}_{3} \mathrm{NaO}_{7}: 584.2373\left[\mathrm{M}^{+}+\mathrm{MeOH}+\mathrm{Na}\right]$; found: 584.2372. All attempts at obtaining correct elemental analysis for this azide in two different analyzers failed.

Photocyclization of 21: A deoxygenated solution of diketone 21 (31 mg, $0.059 \mathrm{mmol})$ in purified $\mathrm{CDCl}_{3}(1.0 \mathrm{~mL})$, was irradiated with a daylight lamp at $308 \mathrm{C}$ for $2 \mathrm{~h}$. The solution was concentrated under reduced pressure and the residue purified by Chromatotron chromatography $\left(\mathrm{Al}_{2} \mathrm{O}_{3}\right.$, hexanes/EtOAc 8:2) to give the isomeric mixture $(3 a S, 4 S, 5 R, 6 S, 6 a R)-6 a-$ (azidomethyl)-4,5,6-tris(benzyloxy)-2-hydroxy-2-methyltetrahydro-2H-cyclopenta[b]furan-3(3aH)-one (28) (17 mg, $0.032 \mathrm{mmol}$, d.r. $1.3: 1,55 \%)$ as a colorless oil: ${ }^{1} \mathrm{H}$ NMR $\left(500 \mathrm{MHz}, \mathrm{CDCl}_{3}\right): \mathrm{d}=1.36(\mathrm{~s}, 3 \mathrm{H}), 1.49(\mathrm{~s}, 3 \mathrm{H})$, 3.09 (d, $J=2.5 \mathrm{~Hz}, 1 \mathrm{H}), 3.12(\mathrm{~d}, J=3.5 \mathrm{~Hz}, 1 \mathrm{H}), 3.46(\mathrm{~d}, J=$ $13.6 \mathrm{~Hz}, 1 \mathrm{H}), 3.78(\mathrm{~d}, J=10.6 \mathrm{~Hz}, 1 \mathrm{H}), 3.79(\mathrm{~d}, J=13.2 \mathrm{~Hz}, 1 \mathrm{H}), 3.89$ (d, $J=4.4 \mathrm{~Hz}, 1 \mathrm{H}), 3.90(\mathrm{~d}, J=12.9 \mathrm{~Hz}, 1 \mathrm{H}), 3.97(\mathrm{dd}, J=3.8,3.5 \mathrm{~Hz}, 1 \mathrm{H})$, 3.99 (dd, $J=5.4,4.7 \mathrm{~Hz}, 1 \mathrm{H}$ ), 4.07 (dd, $J=3.8,3.8 \mathrm{~Hz}, 1 \mathrm{H}$ ), 4.18 (ddd, $J=$ $3.5,2.4,1.1 \mathrm{~Hz}, 1 \mathrm{H}), 4.20(\mathrm{~d}, J=4.1 \mathrm{~Hz}, 1 \mathrm{H}), 4.41-4.70(\mathrm{~m}, 12 \mathrm{H}), 7.21-$ $7.38 \mathrm{ppm}(\mathrm{m}, 30 \mathrm{H}) ;{ }^{13} \mathrm{C}$ NMR $\left(125.7 \mathrm{MHz}, \mathrm{CDCl}_{3}\right): \mathrm{d}=21.3\left(\mathrm{CH}_{3}\right), 22.6$ $\left(\mathrm{CH}_{3}\right), 52.8(\mathrm{CH}), 54.7\left(\mathrm{CH}_{2}\right), 54.7(\mathrm{CH}), 54.9\left(\mathrm{CH}_{2}\right), 71.6\left(\mathrm{CH}_{2}\right), 71.9$ $\left(\mathrm{CH}_{2}\right), 72.3\left(\mathrm{CH}_{2}\right), 72.7\left(\mathrm{CH}_{2}\right), 73.2\left(\mathrm{CH}_{2}\right), 72.2\left(\mathrm{CH}_{2}\right), 82.5(\mathrm{CH}), 83.6$ $(\mathrm{CH}), 85.1(\mathrm{CH}), 85.3(\mathrm{CH}), 87.9(\mathrm{CH}), 88.4(\mathrm{C}), 88.8(\mathrm{CH}), 90.1(\mathrm{C})$, 99.4 (C), 100.3 (C), 127.8-128.7 (30× CH), 136.3 (C), 137.2 (C), 137.3 (C), 137.39 (C), 137.41 (C), 137.5 (C), 207.0 (C), $210.1 \mathrm{ppm}$ (C); I R (film): $\tilde{n}^{\tilde{y}}=$ 3414, 2105, $1767 \mathrm{~cm}^{-1}$; MS (ESI ${ }^{+}$): $\mathrm{m} / \mathrm{z}(\%): 552(100)\left[M^{+}+\mathrm{Na}\right]$; HR MS $\left(\mathrm{ESI}^{+}\right.$): $\mathrm{m} / \mathrm{z}$ calcd for $\mathrm{C}_{30} \mathrm{H}_{31} \mathrm{~N}_{3} \mathrm{NaO}_{6}: 552.2111\left[M^{+}+\mathrm{Na}\right]$; found: 552.2111 . All attempts at obtaining a correct elemental analysis for this azide in two different analyzers failed.
4,8-Anhydro-1-deoxy-5,6-di-O-methyl-7,9-O-isopropylidene-d-glycero-dido-nono-2,3-diulose (22): Prepared from $15(136.0 \mathrm{mg}, 0.50 \mathrm{mmol})$ following the general procedure (Method A) using dimethyl sulfide $(400 \mathrm{~mL}$, $5.4 \mathrm{mmol}$ ). The mixture was stirred at room temperature in the dark for $3.5 \mathrm{~h}$ and concentrated under reduced pressure. The residue was purified by rapid silica gel column chromatography (hexanes/Et0Ac 8:2) to give $22(102.2 \mathrm{mg}, 0.34 \mathrm{mmol}, 67 \%)$ as a yellow oil: $[\mathrm{a}]_{\mathrm{D}}=+107 \mathrm{~cm}^{3} \mathrm{~g}^{-1} \mathrm{dm}^{-1}$ $\left(c=0.58\right.$ in $\left.\mathrm{CHCl}_{3}\right) ;{ }^{1} \mathrm{H} \mathrm{NMR}\left(500 \mathrm{MHz}, \mathrm{CDCl}_{3}\right): \mathrm{d}=1.40(\mathrm{~s}, 3 \mathrm{H}), 1.48(\mathrm{~s}$, $3 \mathrm{H}), 2.28(\mathrm{~s}, 3 \mathrm{H}), 3.34(\mathrm{dd}, J=8.7,8.6 \mathrm{~Hz}, 1 \mathrm{H}), 3.41(\mathrm{~s}, 3 \mathrm{H}), 3.50(\mathrm{~m}$, $1 \mathrm{H}), 3.55$ (s, 3H), 3.58-3.63 (m, 2H), $3.92(\mathrm{dd}, J=10.7,5.3 \mathrm{~Hz}, 1 \mathrm{H}), 4.13$ (ddd, $J=10.1,10.1,5.3 \mathrm{~Hz}, 1 \mathrm{H}), 5.51 \mathrm{ppm}(\mathrm{d}, J=7.6 \mathrm{~Hz}, 1 \mathrm{H}) ;{ }^{13} \mathrm{CNMR}$ $\left(125.7 \mathrm{MHz}, \mathrm{CDCl}_{3}\right): \mathrm{d}=19.0\left(\mathrm{CH}_{3}\right), 23.4\left(\mathrm{CH}_{3}\right), 29.0\left(\mathrm{CH}_{3}\right), 60.0\left(\mathrm{CH}_{3}\right)$, $60.3\left(\mathrm{CH}_{3}\right), 62.5\left(\mathrm{CH}_{2}\right), 67.3(\mathrm{CH}), 71.9(\mathrm{CH}), 74.0(\mathrm{CH}), 81.1(\mathrm{CH}), 81.2$ $(\mathrm{CH}), 99.3(\mathrm{C}), 197.2(\mathrm{C}), 200.3 \mathrm{ppm}(\mathrm{C})$; IR $\left(\mathrm{CHCl}_{3}, 0.2 \mathrm{~mm}\right): \tilde{n}=2999$, $1713 \mathrm{~cm}^{-1}$; MS (ESI ${ }^{+}$): $\mathrm{m} / \mathrm{z}(\%): 357$ (100) $\left[\mathrm{M}^{+}+\mathrm{MeOH}+\mathrm{Na}\right]$; HRMS $\left(\mathrm{ESI}^{+}\right)$): $\mathrm{m} / \mathrm{z}$ calcd for $\mathrm{C}_{15} \mathrm{H}_{26} \mathrm{NaO}_{8}: 357.1525\left[\mathrm{M}^{+}+\mathrm{MeOH}+\mathrm{Na}\right]$; found: 357.1526; elemental analysis calcd (\%) for $\mathrm{C}_{14} \mathrm{H}_{22} \mathrm{O}_{7}$ (302.32): $\mathrm{C} 55.62, \mathrm{H}$ 7.33; found: C 55.57, H 7.34.

Photocyclization of 22 to compound 29: A solution of 22 (16.1 mg, $0.053 \mathrm{mmol})$ in dry $\mathrm{C}_{6} \mathrm{D}_{6}(0.3 \mathrm{~mL})$, was placed in a resonance tube and irradiated with a daylight lamp at 36-40 8C for $2.5 \mathrm{~h}$. The mixture was concentrated under reduced pressure and the residue purified by silica gel column chromatography (hexanes/EtOAc 7:3) to give a mixture of hemiketal diastereomers $29(8.5 \mathrm{mg}, 0.028 \mathrm{mmol}$, d.r. 10:3, 53\%) (major isomer: $\quad(2 S, 3 \mathrm{a} S, 4 S, 5 R, 5 \mathrm{a} S, 9 \mathrm{a} R)$-2-hydroxy-4,5-dimethoxy-2,7,7trimethyltetrahydrofuro $\left[2^{\prime}, 3^{\prime}: 2,3\right]$ cyclopenta[1,2-d][1,3]dioxin-3(2H)-one) as a colorless oil: $[\mathrm{a}]_{\mathrm{D}}=+29 \mathrm{~cm}^{3} \mathrm{~g}^{-1} \mathrm{dm}^{-1}\left(\mathrm{c}=0.50, \mathrm{CHCl}_{3}\right) ;{ }^{1} \mathrm{H} \mathrm{N} \mathrm{MR}$ $\left(500 \mathrm{MHz} \mathrm{CDCl}_{3}\right.$;): $\mathrm{d}$ (major isomer) $=1.36(\mathrm{~s}, 3 \mathrm{H}), 1.47(\mathrm{~s}, 3 \mathrm{H}), 1.48(\mathrm{~s}$ $3 \mathrm{H}$ ), 2.99 (d, $J=2.2 \mathrm{~Hz}, 1 \mathrm{H}), 3.30(\mathrm{~s}, 3 \mathrm{H}), 3.42$ (s, $3 \mathrm{H}), 3.76$ (dd, $J=1.6$, $1.3 \mathrm{~Hz}, 1 \mathrm{H}$ ), 3.89 (d, $J=12.3 \mathrm{~Hz}, 1 \mathrm{H}$ ), 3.92 (ddd, $J=2.2,1.6,1.3 \mathrm{~Hz}, 1 \mathrm{H}$ ), $4.05(\mathrm{~d}, J=12.3 \mathrm{~Hz}, 1 \mathrm{H}), 4.11 \mathrm{ppm}(\mathrm{dd}, J=1.3,1.3 \mathrm{~Hz}, 1 \mathrm{H}) ;{ }^{13} \mathrm{C} \mathrm{N} \mathrm{MR}$ $\left(125.7 \mathrm{MHz}, \mathrm{CDCl}_{3}\right)$ : d (major isomer) $=22.3\left(\mathrm{CH}_{3}\right), 22.8\left(\mathrm{CH}_{3}\right), 25.5$ $\left(\mathrm{CH}_{3}\right), 54.9(\mathrm{CH}), 56.0\left(\mathrm{CH}_{3}\right), 57.2\left(\mathrm{CH}_{3}\right), 66.4\left(\mathrm{CH}_{2}\right), 79.0(\mathrm{CH}), 87.9$ $(\mathrm{CH}), 88.0(\mathrm{C}), 88.8(\mathrm{CH}), 99.7(\mathrm{C}), 99.8(\mathrm{C}), 210.8 \mathrm{ppm}(\mathrm{C})$; IR $\left(\mathrm{CHCl}_{3}\right.$, $0.2 \mathrm{~mm}): \tilde{n}=3581,3016,1770 \mathrm{~cm}^{-1}$; MS $\left(\mathrm{ESI}^{+}\right): \mathrm{m} / \mathrm{z}(\%): 325(100)\left[\mathrm{M}^{+}\right.$ $+\mathrm{Na}$ ]; HRMS (ESI ${ }^{+}$): $\mathrm{m} / \mathrm{z}$ calcd for $\mathrm{C}_{14} \mathrm{H}_{22} \mathrm{NaO}_{7}: 325.1263\left[M^{+}+\mathrm{Na}\right]$; found: 325.1252; elemental analysis calcd (\%) for $\mathrm{C}_{14} \mathrm{H}_{22} \mathrm{O}_{7}$ (302.32): C 55.62, H 7.33; found: C 55.73, H 7.00.

4,8-Anhydro-7,9-bis-O-(tert-butyldimethylsilyl)-1-deoxy-5,6-di-O-methyld-glycero-d-ido-nono-2,3-diulose (23): Prepared from $16 \quad(51 \mathrm{mg}$, $0.12 \mathrm{mmol}$ ) following the general procedure (Method A) using dimethyl sulfide ( $15 \mathrm{~mL}, 0.2 \mathrm{mmol})$. The mixture was stirred at room temperature in the dark for $2 \mathrm{~h}$ and concentrated under reduced pressure. The residue was purified by rapid silica gel column chromatography (hexanes/EtOAc 1:1) to give 23 (40 mg, $0.09 \mathrm{mmol}, 75 \%$ ) as a yellow oil: $[\mathrm{a}]_{\mathrm{D}}=+74 \mathrm{~cm}^{3} \mathrm{~g}^{-1} \mathrm{dm}^{-1}$ $\left(c=0.82\right.$ in $\left.\mathrm{CHCl}_{3}\right) ;{ }^{1} \mathrm{H} \mathrm{NMR}\left(500 \mathrm{MHz}, \mathrm{CDCl}_{3}\right): \mathrm{d}=0.02(\mathrm{~s}, 3 \mathrm{H}), 0.03$ (s, $3 \mathrm{H}), 0.09(\mathrm{~s}, 3 \mathrm{H}), 0.10(\mathrm{~s}, 3 \mathrm{H}), 0.87(\mathrm{~s}, 9 \mathrm{H}), 0.88(\mathrm{~s}, 9 \mathrm{H})$,

2.29 (s, $3 \mathrm{H}), 3.17$ (dd, $J=8.5,8.2 \mathrm{~Hz}, 1 \mathrm{H}), 3.38$ (s, $3 \mathrm{H}), 3.51$ (s, $3 \mathrm{H}), 3.55$ (dd, $J=8.5,7.3 \mathrm{~Hz}, 1 \mathrm{H}$ ), 3.58 (dd, $J=9.1,8.2 \mathrm{~Hz}, 1 \mathrm{H}$ ), 3.75 (dd, $J=11.7$, $3.5 \mathrm{~Hz}, 1 \mathrm{H}$ ), 3.81 (dd, $J=11.7,1.9 \mathrm{~Hz}, 1 \mathrm{H}$ ), 4.02 (ddd, $J=9.2,3.4,1.9 \mathrm{~Hz}$,

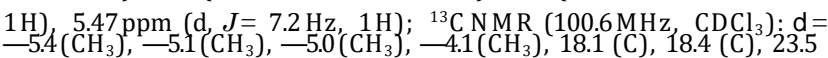
$\left(\mathrm{CH}_{3}\right), 25.90\left(3 \times \mathrm{CH}_{3}\right), 25.95\left(3 \times \mathrm{CH}_{3}\right), 58.9\left(\mathrm{CH}_{3}\right), 60.6\left(\mathrm{CH}_{3}\right), 62.3$ $\left(\mathrm{CH}_{2}\right), 69.2(\mathrm{CH}), 72.1(\mathrm{CH}), 77.1(\mathrm{CH}), 81.9(\mathrm{CH}), 83.9(\mathrm{CH}), 197.6(\mathrm{C})$, $201.3 \mathrm{ppm}$ (C); IR (film): $\tilde{n=} 2955,1711 \mathrm{~cm}^{-1}$; MS (EI): $m / z(\%): 491$ (1) $\left[M^{+}+\mathrm{H}\right], 433$ (87); HRMS (EI): $m / z$ calcd for $\mathrm{C}_{23} \mathrm{H}_{47} \mathrm{O}_{7} \mathrm{Si}_{2}: 491.2860\left[M^{+}\right.$ $+\mathrm{H}$ ]; found: 491.2875; elemental analysis calcd (\%) for $\mathrm{C}_{23} \mathrm{H}_{46} \mathrm{O}_{7} \mathrm{Si}_{2}$ (490.78):C 56.29, H 9.45; found: C 56.30, H 9.45.

Photocyclization of 23: A solution of diketone $23(28 \mathrm{mg}, 0.057 \mathrm{mmol})$ in purified $\mathrm{CDCl}_{3}(0.7 \mathrm{~mL})$, was placed in a resonance tube and irradiated with a daylight lamp at $308 \mathrm{C}$ for $3 \mathrm{~h}$. The mixture was concentrated under reduced pressure and the residue purified by Chromatotron chromatography $\left(\mathrm{Al}_{2} \mathrm{O}_{3}\right.$, toluene as eluent) to give $(2 S, 3 \mathrm{a} S, 4 S, 5 R, 6 S, 6 \mathrm{a} R)-6$ [(tert-butyldimethylsilyl)oxy]-6a-[(tert-butyldimethylsilyl)oxy]methyl-2-hydroxy-4,5-dimethoxy-2-methyltetrahydro- $2 H$-cyclopenta[b]furan-3(3a $H)$ one (30) (17.5 mg, $0.036 \mathrm{mmol}, 63 \%$ ) as a colorless oil: $[\mathrm{a}]_{\mathrm{D}}=+54 \mathrm{~cm}^{3} \mathrm{~g}$ ${ }^{1} \mathrm{dm}^{-1}\left(c=2.10\right.$ in $\left.\mathrm{CHCl}_{3}\right) ;{ }^{1} \mathrm{H} \mathrm{NMR}\left(500 \mathrm{MHz}, \mathrm{CDCl}_{3}\right): \mathrm{d}=$ $0.091(\mathrm{~s}, 3 \mathrm{H}), 0.099(\mathrm{~s}, 3 \mathrm{H}), 0.13(\mathrm{~s}, 3 \mathrm{H}), 0.14(\mathrm{~s}, 3 \mathrm{H}), 0.89(\mathrm{~s}, 9 \mathrm{H}), 0.92$ 
(s, 9H), 1.39 (d, $J=0.9 \mathrm{~Hz}, 3 \mathrm{H}), 2.95$ (d, $J=4.4 \mathrm{~Hz}, 1 \mathrm{H}), 3.39$ (s, 3H), 3.43 (s, $3 \mathrm{H}$ ), 3.53 (dd, $J=5.4,5.4 \mathrm{~Hz}, 1 \mathrm{H}), 3.69$ (dd, $J=5.0,4.4 \mathrm{~Hz}, 1 \mathrm{H}$ ), $3.77(\mathrm{~d}, J=11.4 \mathrm{~Hz}, 1 \mathrm{H}), 3.85(\mathrm{~d}, J=11.4 \mathrm{~Hz}, 1 \mathrm{H}), 3.99(\mathrm{~d}, J=5.7 \mathrm{~Hz}$, $1 \mathrm{H}), 5.11 \mathrm{ppm}(\mathrm{d}, J=0.9 \mathrm{~Hz}, 1 \mathrm{H}) ;{ }^{13} \mathrm{CNMR}\left(100.6 \mathrm{MHz}, \mathrm{CDCl}_{3}\right): \mathrm{d}=$ $-5.7\left(\mathrm{CH}_{3}\right),-5.6\left(\mathrm{CH}_{3}\right),-5.1\left(\mathrm{CH}_{3}\right),-4.8\left(\mathrm{CH}_{3}\right), 18.0(\mathrm{C}), 18.4(\mathrm{C}), 20.3$ $\left(\mathrm{CH}_{3}\right), 25.6\left(3 \times \mathrm{CH}_{3}\right), 25.8\left(3 \times \mathrm{CH}_{3}\right), 51.3(\mathrm{CH}), 57.6\left(\mathrm{CH}_{3}\right), 58.4\left(\mathrm{CH}_{3}\right)$ $65.9\left(\mathrm{CH}_{2}\right), 81.6(\mathrm{CH}), 72.1(\mathrm{CH}), 84.5(\mathrm{CH}), 89.4(\mathrm{CH}), 90.1(\mathrm{CH}), 98.7$ (CH), 208.5 ppm (C); IR (film): $\tilde{n}=3368,2931,1765 \mathrm{~cm}^{-1}$; MS (EI): $\mathrm{m} / \mathrm{z}$ (\%): 473(2) [ $M^{+}-\mathrm{OH}$ ], 433(7); HRMS (EI): $m /$ zcalcd for $\mathrm{C}_{23} \mathrm{H}_{45} \mathrm{O}_{6} \mathrm{Si}_{2}$ : 473.2755[ $\left.\mathrm{M}^{+}-\mathrm{OH}\right]$; found: 473.2761; elemental analysis calcd (\%) for $\mathrm{C}_{23} \mathrm{H}_{46} \mathrm{O}_{7} \mathrm{Si}_{2}$ (490.78): C 56.29, H 9.45; found: C 56.21, H 9.22. 7-O-Acetyl-4,8-anhydro-9-O-(tert-butyldimethylsilyl)-1-deoxy-5,6-di-Omethyl-d-glycero-d-ido-nono-2,3-diulose (24): Prepared from 17 (176.7 $\mathrm{mg}, 0.46 \mathrm{mmol}$ ) following the general procedure (Method A) using dimethyl sulfide $(267 \mathrm{~mL}, 3.64 \mathrm{mmol})$. The mixture was stirred at room temperature in the dark for $2 \mathrm{~h}$ and concentrated under reduced pressure. The residue was purified by rapid silica gel column chromatography (hexanes/EtOAc 1:1) to give $24(139.0 \mathrm{mg}, 0.33 \mathrm{mmol}, 73 \%)$ as a yellow oil: $[\mathrm{a}]_{\mathrm{D}}=+76 \mathrm{~cm}^{3} \mathrm{~g}^{-1} \mathrm{dm}^{-1}\left(c=0.59 \mathrm{in} \mathrm{CHCl}_{3}\right) ;{ }^{1} \mathrm{H} \mathrm{N} \mathrm{MR}\left(500 \mathrm{MHz}, \mathrm{CDCl}_{3}\right)$ : $\mathrm{d}=0.02(\mathrm{~s}, 6 \mathrm{H}), 0.87(\mathrm{~s}, 9 \mathrm{H}), 2.06(\mathrm{~s}, 3 \mathrm{H}), 2.30(\mathrm{~s}, 3 \mathrm{H}), 3.35(\mathrm{~s}, 3 \mathrm{H}), 3.46$ (dd, $J=6.8,6.6 \mathrm{~Hz}, 1 \mathrm{H}$ ), 3.50 (s, $3 \mathrm{H}$ ), 3.65 (dd, $J=11.4,4.7 \mathrm{~Hz}, 1 \mathrm{H}$ ), 3.74 (dd, $J=11.4,3.2 \mathrm{~Hz}, 1 \mathrm{H}$ ), 3.80 (dd, $J=6.8,6.6 \mathrm{~Hz}, 1 \mathrm{H}$ ), 4.16 (ddd, $J=$ $8.8,4.7,3.2 \mathrm{~Hz}, 1 \mathrm{H}), 4.96(\mathrm{dd}, J=8.8,6.6 \mathrm{~Hz}, 1 \mathrm{H}), 5.47 \mathrm{ppm}(\mathrm{d}, J=$ $6.6 \mathrm{~Hz}, 1 \mathrm{H}) ;{ }^{13} \mathrm{C} \mathrm{NMR}\left(125.7 \mathrm{MHz}, \mathrm{CDCl}_{3}\right): \mathrm{d}=-5.5\left(2 \times \mathrm{CH}_{3}\right), 18.3(\mathrm{C})$, $21.0\left(\mathrm{CH}_{3}\right), 23.5\left(\mathrm{CH}_{3}\right), 25.8\left(3 \times \mathrm{CH}_{3}\right), 58.9\left(\mathrm{CH}_{3}\right), 59.6\left(\mathrm{CH}_{3}\right), 63.0$ $\left(\mathrm{CH}_{2}\right), 68.8(\mathrm{CH}), 72.9(\mathrm{CH}), 74.6(\mathrm{CH}), 79.6(2 \times \mathrm{CH}), 169.7(\mathrm{C}), 197.1$ (C), $198.5 \mathrm{ppm}(\mathrm{C})$; IR $\left(\mathrm{CHCl}_{3}, 0.2 \mathrm{~mm}\right): \tilde{n^{-}}=2933,1738,1715 \mathrm{~cm}^{-1}$; MS $\left(\mathrm{ESI}^{+}\right): \mathrm{m} / \mathrm{z}(\%): 473(100)\left[\mathrm{M}^{+}+\mathrm{MeOH}+\mathrm{Na}\right] ; \mathrm{HRMS}\left(\mathrm{ESI}^{+}\right): \mathrm{m} / z$ calcd for $\mathrm{C}_{20} \mathrm{H}_{38} \mathrm{NaO}_{9} \mathrm{Si}: 473.2183\left[M^{+}+\mathrm{MeOH}+\mathrm{Na}\right.$ ]; found: 473.2182; elemental analysis calcd (\%) for $\mathrm{C}_{19} \mathrm{H}_{34} \mathrm{O}_{8} \mathrm{Si}$ (418.55): $\mathrm{C} 54.52, \mathrm{H}$ 8.19; found: $\mathrm{C}$ 54.53, H 8.19.

Photocyclization of 24: A deoxygenated solution of diketone 24 (41.1 mg, $0.098 \mathrm{mmol})$ in dry $\mathrm{C}_{6} \mathrm{D}_{6}(0.3 \mathrm{~mL})$, was placed in a resonance tube and irradiated with a daylight lamp at 36-40 8C for $3 \mathrm{~h}$. The solution was concentrated under reduced pressure and the residue purified by silica gel column chromatography (hexanes/Et0Ac 7:3) to give a mixture of hemiketal diastereomers $31(25.4 \mathrm{mg}, 0.061 \mathrm{mmol}$, d.r., 10:3, 62\%) (major isomer: $(2 S, 3 \mathrm{a} S, 4 S, 5 R, 6 S, 6 \mathrm{a} R)-6 a-[($ tert-butyldimethylsilyl)oxy]- methyl-5ethoxy-2-hydroxy-4-methoxy-2-methyl-3-oxohexahydro- $2 \mathrm{H}$-cyclopenta[b]furan-6-yl acetate) as a colorless oil: [a] $=$ + $56 \mathrm{~cm}^{3} \mathrm{~g}^{-1} \mathrm{dm}^{-1}$ $\left(c=0.59 \mathrm{in} \mathrm{CHCl}_{3}\right) ;{ }^{1} \mathrm{H} \mathrm{NMR}\left(500 \mathrm{MHz} \mathrm{CDCl}_{3}\right): \mathrm{d}$ (major isomer) $=0.15$ (s, 6H), $0.92(\mathrm{~s}, 9 \mathrm{H}), 1.40$ (d, $J=1.0 \mathrm{~Hz}, 3 \mathrm{H}), 2.08$ (s, 3H), 3.11 (dd, $J=$ 2.1, $0.9 \mathrm{~Hz}, 1 \mathrm{H}$ ), 3.34 (s, $3 \mathrm{H}$ ), 3.38 (s, $3 \mathrm{H}$ ), 3.74 (ddd, $J=2.3,2.3,0.8 \mathrm{~Hz}$, $1 \mathrm{H}$ ), 3.77 (d, $J=10.7 \mathrm{~Hz}, 1 \mathrm{H}$ ), 3.80 (d, $J=10.7 \mathrm{~Hz}, 1 \mathrm{H}$ ), 3.87 (ddd, $J=$ 2.1, 2.1, $0.9 \mathrm{~Hz}, 1 \mathrm{H}), 4.91(\mathrm{~d}, J=1.0 \mathrm{~Hz}, 1 \mathrm{H}), 5.21 \mathrm{ppm}(\mathrm{dd}, J=2.5$, $1.0 \mathrm{~Hz}, 1 \mathrm{H}) ;{ }^{13} \mathrm{C} \mathrm{NMR}\left(125.7 \mathrm{M} \mathrm{Hz}, \mathrm{CDCl}_{3}\right): \mathrm{d}$ (major isomer) $=-5.64$ $\left(\mathrm{CH}_{3}\right),-5.55\left(\mathrm{CH}_{3}\right), 18.4(\mathrm{C}), 20.0\left(\mathrm{CH}_{3}\right), 20.8\left(\mathrm{CH}_{3}\right), 25.8\left(3 \times \mathrm{CH}_{3}\right)$, $52.7(\mathrm{CH}), 57.2\left(\mathrm{CH}_{3}\right), 57.3\left(\mathrm{CH}_{3}\right), 64.7\left(\mathrm{CH}_{2}\right), 80.5(\mathrm{CH}), 86.46(\mathrm{CH})$, $86.58(\mathrm{CH}), 90.7(\mathrm{C}), 98.7(\mathrm{C}), 169.6(\mathrm{C}), 207.0 \mathrm{ppm}(\mathrm{C})$; IR $\left(\mathrm{CHCl}_{3}\right.$, $0.2 \mathrm{~mm}): \tilde{n^{2}}=2933,1766,1742 \mathrm{~cm}^{-1} ; \mathrm{MS}\left(\mathrm{ESI}^{+}\right): \mathrm{m} / \mathrm{z}(\%): 441(100)\left[\mathrm{M}^{+}\right.$ $+\mathrm{Na}]$; HRMS (ESI ${ }^{+}$): $\mathrm{m} / \mathrm{z}$ calcd for $\mathrm{C}_{19} \mathrm{H}_{34} \mathrm{NaO}_{8} \mathrm{Si}: 441.1921\left[M^{+}+\mathrm{Na}\right]$; found: 441.1920; elemental analysis calcd (\%) for $\mathrm{C}_{19} \mathrm{H}_{34} \mathrm{O}_{8} \mathrm{Si}$ (418.55): C 54.52, H 8.19; found: C 54.55, H 8.14.

4,8-Anhydro-9-O-(tert-butyldiphenylsilyl)-1,5-dideoxy-6,7-di-O-methyl-dmanno-nono-2,3-diulose (38): Prepared from 32 (70 $\mathrm{mg}, 0.155 \mathrm{mmol}$ ) following the general procedure (Method B) using $\mathrm{NaIO}_{4}$ (132 mg, $0.62 \mathrm{mmol})$ and $\mathrm{RuO}_{2} \cdot x \mathrm{H}_{2} \mathrm{O}(1.1 \mathrm{mg})$. The reaction mixture was stirred at room temperature in the dark for $1 \mathrm{~h}$. The residue was purified by rapid silica gel column chromatography (hexanes/EtOAc 1:1) to give $38(47 \mathrm{mg}$, $0.097 \mathrm{mmol}, 63 \%)$ as a yellow oil: [a $]_{\mathrm{D}}=+13 \mathrm{~cm}^{3} \mathrm{~g}^{-1} \mathrm{dm}^{-1}(\mathrm{c}=0.94 \mathrm{in}$ $\left.\mathrm{CHCl}_{3}\right) ;{ }^{1} \mathrm{H} \mathrm{NMR}\left(500 \mathrm{MHz}, \mathrm{C}_{6} \mathrm{D}_{6}\right): \mathrm{d}=1.18(\mathrm{~s}, 9 \mathrm{H}), 1.60$ (ddd, $J=13.9$, 9.8, $6.0 \mathrm{~Hz}, 1 \mathrm{H}$ ), $1.86(\mathrm{~s}, 3 \mathrm{H}), 2.22$ (ddd, $J=13.9,4.4,3.8 \mathrm{~Hz}$,

$1 \mathrm{H}), 3.11$ (s, 3H), 3.23 (dd, $J=7.9,7.9 \mathrm{~Hz}, 1 \mathrm{H}$ ), 3.35 (s, $3 \mathrm{H}), 3.41$ (ddd, $J=9.8,7.6,4.4 \mathrm{~Hz}, 1 \mathrm{H}$ ), 3.77 (ddd, $J=7.9,4.4,2.8 \mathrm{~Hz}, 1 \mathrm{H}$ ), 3.98 (dd, $J=$ $11.0,4.4 \mathrm{~Hz}, 1 \mathrm{H}$ ), 4.02 (dd, $J=11.0,2.8 \mathrm{~Hz}, 1 \mathrm{H}$ ), 4.89 (dd, $J=6.0,3.8 \mathrm{~Hz}$, $1 \mathrm{H}), 7.21-7.86 \mathrm{ppm}(\mathrm{m}, 10 \mathrm{H}) ;{ }^{13} \mathrm{CNMR}\left(125.7 \mathrm{MHz}, \mathrm{C}_{6} \mathrm{D}_{6}\right): \mathrm{d}=19.5(\mathrm{C})$ $24.4\left(\mathrm{CH}_{3}\right), 27.0\left(3 \times \mathrm{CH}_{3}\right), 28.6\left(\mathrm{CH}_{2}\right), 56.6\left(\mathrm{CH}_{3}\right), 59.7\left(\mathrm{CH}_{3}\right), 63.6$ $\left(\mathrm{CH}_{2}\right), 72.5(\mathrm{CH}), 77.6(\mathrm{CH}), 78.6(\mathrm{CH}), 79.0(\mathrm{CH}), 127.8-136.2(10 \times$
CH), 133.8 (C), 134.0 (C), 198.9 (C), 199.9 ppm (C); IR (film): $\tilde{n^{2}}$ 2938, $1722 \mathrm{~cm}^{-1}$; MS (ESI ${ }^{+}$): $m / z(\%): 539(100)\left[M^{+}+\mathrm{MeOH}+\mathrm{Na}\right]$; HRMS $\left(\mathrm{ESI}^{+}\right.$): $\mathrm{m} / \mathrm{z}$ calcd for $\mathrm{C}_{28} \mathrm{H}_{40} \mathrm{NaO}_{7} \mathrm{Si}: 539.2441\left[\mathrm{M}^{+}+\mathrm{MeOH}+\mathrm{Na}\right]$; found: 539.2440; elemental analysis calcd (\%) for $\mathrm{C}_{27} \mathrm{H}_{36} \mathrm{O}_{6} \mathrm{Si}$ (484.66): C 66.91, $\mathrm{H}$ 7.49; found: C 67.09, H 7.52.

Photocyclization of 38: A solution of diketone 38 ( $48 \mathrm{mg}, 0.102 \mathrm{mmol})$ in dry $\mathrm{C}_{6} \mathrm{D}_{6}(1.0 \mathrm{~mL})$, was placed in a resonance tube and irradiated with a daylight lamp at $308 \mathrm{C}$. The reaction was monitored by ${ }^{1} \mathrm{H}$ N M R spectroscopy, complete consumption of starting material was observed after $9 \mathrm{~h}$. Then, the reaction mixture was heated at $608 \mathrm{C}$ for $5 \mathrm{~h}$ and concentrated under reduced pressure. The residue was solved in $\mathrm{CH}_{2} \mathrm{Cl}_{2}(0.82 \mathrm{~mL})$ cooled to $08 \mathrm{C}$ and acetylated by adding $\mathrm{Ac}_{2} \mathrm{O}(29 \mathrm{~mL}, 0.306 \mathrm{mmol})$ and D M A P ( $37 \mathrm{mg}, 0.306 \mathrm{mmol}$ ) followed by stirring at room temperature for 2 $h$. The reaction mixture was concentrated under reduced pressure and the residue partially resolved by silica gel column chromatography (hexanes/EtOAc 8:2) to give $(2 R, 3 \mathrm{a} S, 5 R, 6 S, 6 \mathrm{a} R)-6 \mathrm{a}$-[(tert-butyldiphenylsilyl)oxy]methyl-5,6-dimethoxy-2-methyl-3-oxohexahydro- $2 H$-cyclopenta $[b]$ furan-2-yl acetate $(44)$ and $(2 S, 3 a R, 5 R, 6 S, 6 a S)-6 a-[($ tert-butyldiphenylsilyl)oxy]methyl-5,6-dimethoxy-2-methyl-3-oxohexahydro- $2 \mathrm{H}$-cyclopenta $[b]$ furan-2-yl acetate (45) (21 mg, $0.040 \mathrm{mmol}$, d.r. 1:1,39\%). The sensitive photoenol intermediate was detected by N M R spectroscopy but in this case could not be obtained in pure state. Only clearly distinguish- ed signals from the reaction mixture are reported: ${ }^{1} \mathrm{H} \mathrm{N} \mathrm{MR}\left(500 \mathrm{MHz}, \mathrm{C}_{6} \mathrm{D}_{6}\right)$ : $\mathrm{d}=1.25(\mathrm{~s}, 9 \mathrm{H}), 1.75(\mathrm{~s}, 3 \mathrm{H}), 2.85(\mathrm{~s}, 3 \mathrm{H}), 3.00(\mathrm{~s}, 3 \mathrm{H}), 4.70(\mathrm{~d}$,

$J=18.6 \mathrm{~Hz}, 1 \mathrm{H}$ ), $4.82(\mathrm{~d}, J=18.6 \mathrm{~Hz}, 1 \mathrm{H}$ ), 5.28 (dd, $J=7.5,7.5 \mathrm{~Hz}, 1 \mathrm{H}$ ), $6.66 \mathrm{ppm}(\mathrm{br} \mathrm{s}, 1 \mathrm{H}, \mathrm{OH}) ;{ }^{13} \mathrm{C} \mathrm{NMR}\left(125.7 \mathrm{MHz}, \mathrm{C}_{6} \mathrm{D}_{6}\right): \mathrm{d}=19.6(\mathrm{C}), 22.3$ $\left(\mathrm{CH}_{3}\right), 27.0\left(3 \times \mathrm{CH}_{3}\right), 57.6\left(\mathrm{CH}_{3}\right), 59.3\left(\mathrm{CH}_{3}\right), 69.5\left(\mathrm{CH}_{2}\right), 111.6(\mathrm{CH})$, 149.2 (C), 193.9 (C), $207.5 \mathrm{ppm}(\mathrm{C})$.

Compound 44: colorless oil. [a $]_{\mathrm{D}}=-2 \mathrm{~cm}^{3} \mathrm{~g}^{-1} \mathrm{dm}^{-1}(c=0.54 \mathrm{inCHCl})$; $_{3}$ ${ }^{1} \mathrm{H} \mathrm{NMR}\left(500 \mathrm{MHz}, \mathrm{CDCl}_{3}\right): \mathrm{d}=1.06(\mathrm{~s}, 9 \mathrm{H}), 1.55(\mathrm{~s}, 3 \mathrm{H}), 1.75(\mathrm{~s}, 3 \mathrm{H})$, 1.99 (dddd, $J=13.9,4.4,4.4,1.3 \mathrm{~Hz}, 1 \mathrm{H}$ ), 2.30 (ddd, $J=13.9,10.4,5.4 \mathrm{~Hz}$, $1 \mathrm{H}$ ), 3.23 (dd, $J=10.4,4.4 \mathrm{~Hz}, 1 \mathrm{H}$ ), 3.27 (s, $3 \mathrm{H}$ ), 3.47 (s, $3 \mathrm{H}$ ), 3.73 (br d, $J=4.1 \mathrm{~Hz}, 1 \mathrm{H}), 3.85(\mathrm{~d}, J=10.1 \mathrm{~Hz}, 1 \mathrm{H}), 3.88(\mathrm{ddd}, J=4.7,4.7,4.7 \mathrm{~Hz}$, $1 \mathrm{H}), \quad 3.91(\mathrm{~d}, J=10.1 \mathrm{~Hz}, 1 \mathrm{H}), 7.36-7.79 \mathrm{ppm}(\mathrm{m}, 10 \mathrm{H}) ;{ }^{13} \mathrm{CNMR}$ $\left(100.6 \mathrm{MHz}, \mathrm{CDCl}_{3}\right): \mathrm{d}=19.3(\mathrm{C}), 20.7\left(\mathrm{CH}_{3}\right), 23.0\left(\mathrm{CH}_{3}\right), 26.9\left(3 \times \mathrm{CH}_{3}\right)$, $31.7\left(\mathrm{CH}_{2}\right), 46.4(\mathrm{CH}), 56.4\left(\mathrm{CH}_{3}\right), 58.9\left(\mathrm{CH}_{3}\right), 64.4\left(\mathrm{CH}_{2}\right), 82.7(\mathrm{CH})$, $90.6(\mathrm{CH}), 93.1(\mathrm{C}), 101.9(\mathrm{C}), 127.6-135.8(10 \times \mathrm{CH}), 133.42(\mathrm{C}), 133.45$

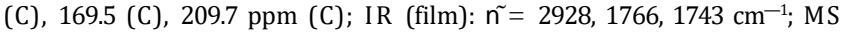
$\left(\mathrm{ESI}^{+}\right): \mathrm{m} / \mathrm{z}(\%): 549(100)\left[\mathrm{M}^{+}+\mathrm{Na}\right]$; HRMS $\left(\mathrm{ESI}^{+}\right): \mathrm{m} / \mathrm{z}$ calcd for $\mathrm{C}_{29} \mathrm{H}_{38} \mathrm{NaO}_{7} \mathrm{Si}$ : $549.2285\left[M^{+}+\mathrm{Na}\right]$; found: 549.2285; elemental analysis calcd (\%) for $\mathrm{C}_{29} \mathrm{H}_{38} \mathrm{O}_{7} \mathrm{Si}$ (526.69): C 66.13, $\mathrm{H}$ 7.27; found: $\mathrm{C} 66.40, \mathrm{H} \quad 7.61$.

Compound 45: colorless oil, [a $]_{\mathrm{D}}=-15 \mathrm{~cm}^{3} \mathrm{~g}^{-1} \mathrm{dm}^{-1}\left(c=0.56\right.$ in $\left.\mathrm{CHCl}_{3}\right)$; ${ }^{1} \mathrm{H} \mathrm{NMR}\left(500 \mathrm{MHz}, \mathrm{CDCl}_{3}\right): \mathrm{d}=1.07(\mathrm{~s}, 9 \mathrm{H}), 1.50$ (s, 3H), 1.77 (ddd, $J=$ 12.8, 9.8, $9.8 \mathrm{~Hz}, 1 \mathrm{H}$ ), 1.86 (s, $3 \mathrm{H}$ ), 2.27 (ddd, $J=12.9,6.3,2.2 \mathrm{~Hz}, 1 \mathrm{H}$ ), 3.31 (dd, $J=10.1,2.2 \mathrm{~Hz}, 1 \mathrm{H}), 3.35$ (s, $3 \mathrm{H}), 3.46$ (s, $3 \mathrm{H}), 3.49(\mathrm{~m}, 1 \mathrm{H})$, 3.77 (d, $J=10.1 \mathrm{~Hz}, 1 \mathrm{H}), 3.85$ (d, $J=9.8 \mathrm{~Hz}, 1 \mathrm{H}), 3.92$ (d, $J=7.6 \mathrm{~Hz}$, $1 \mathrm{H}), 7.36-7.69 \mathrm{ppm}(\mathrm{m}, 10 \mathrm{H}) ;{ }^{13} \mathrm{C} \mathrm{NMR}\left(100.6 \mathrm{MHz}, \mathrm{CDCl}_{3}\right): \mathrm{d}=19.3$ (C), $20.5\left(\mathrm{CH}_{3}\right), 21.9\left(\mathrm{CH}_{3}\right), 26.9\left(3 \times \mathrm{CH}_{3}\right), 30.4\left(\mathrm{CH}_{2}\right), 46.8(\mathrm{CH}), 57.5$ $\left(\mathrm{CH}_{3}\right), 59.5\left(\mathrm{CH}_{3}\right), 65.4\left(\mathrm{CH}_{2}\right), 84.5(\mathrm{CH}), 84.8(\mathrm{CH}), 88.5(\mathrm{C}), 101.2(\mathrm{C})$, 127.7-135.7 (10 × CH), $132.9(\mathrm{C}), 133.0(\mathrm{C}), 169.5(\mathrm{C}), 211.0 \mathrm{ppm}(\mathrm{C})$; IR (film): $\tilde{n}=2931,1768,1744 \mathrm{~cm}^{-1}$; MS $\left(\mathrm{ESI}^{+}\right): \mathrm{m} / \mathrm{z}(\%): 549$ (100) $\left[\mathrm{M}^{+}\right.$ $+\mathrm{Na}]$; HRMS $\left(\mathrm{ESI}^{+}\right): \mathrm{m} / \mathrm{z}$ calcd for $\mathrm{C}_{29} \mathrm{H}_{38} \mathrm{NaO}_{7} \mathrm{Si}: 549.2285\left[M^{+}+\mathrm{Na}\right]$; found: 549.2288; elemental analysis calcd (\%) for $\mathrm{C}_{29} \mathrm{H}_{38} \mathrm{O}_{7} \mathrm{Si}$ (526.69): $\mathrm{C}$ 66.13, H 7.27; found: C 66.11, H 7.58.

4,8-Anhydro-1,6-dideoxy-5,7,9-tri-O-methyl-d-altro-nono-2,3-diulose (39): Prepared from $33(111 \mathrm{mg}, 0.486 \mathrm{mmol}$ ) following the general procedure (Method A) using dimethyl sulfide ( $71 \mathrm{~mL}, 0.970 \mathrm{mmol})$. The mixture was stirred at room temperature in the dark for $6 \mathrm{~h}$ and concentrat- ed under reduced pressure. The residue was purified by rapid silica gel column chromatography (hexanes/EtOAc $7: 3 ! 6: 4)$ to give $39(83 \mathrm{mg}$, $0.315 \mathrm{mmol}, 65 \%)$ as a yellow oil: $[\mathrm{a}]_{\mathrm{D}}=+126 \mathrm{~cm}^{3} \mathrm{~g}^{-1} \mathrm{dm}^{-1}(c=1.3 \mathrm{in}$ $\left.\mathrm{CHCl}_{3}\right) ;{ }^{1} \mathrm{H} \mathrm{NMR}\left(500 \mathrm{MHz}, \mathrm{CDCl}_{3}\right): \mathrm{d}=1.72$ (ddd, $J=13.2,8.8,7.6 \mathrm{~Hz}$, $1 \mathrm{H}$ ), 2.19 (ddd, $J=13.2,5.1,3.8 \mathrm{~Hz}, 1 \mathrm{H}$ ), 2.26 (s, $3 \mathrm{H}$ ), 3.27 (s, $3 \mathrm{H}$ ), 3.35 (s, $3 \mathrm{H}$ ), 3.36 (m, $1 \mathrm{H}$ ), 3.40 (s, $3 \mathrm{H}$ ), 3.55 (dd, $J=10.7,4.7 \mathrm{~Hz}, 1 \mathrm{H}$ ), 3.64 (dd, $J$ $=10.7,2.2 \mathrm{~Hz}, 1 \mathrm{H}$ ), 3.87 (ddd, $J=10.7,6.6,3.8 \mathrm{~Hz}, 1 \mathrm{H}$ ), 4.10 (ddd, $J=9.2$, 4.7, $2.2 \mathrm{~Hz}, 1 \mathrm{H}), 5.38 \mathrm{ppm}(\mathrm{d}, J=6.9 \mathrm{~Hz}, 1 \mathrm{H}) ;{ }^{13} \mathrm{C}$ NMR $(100.6 \mathrm{MHz}$, $\left.\mathrm{CDCl}_{3}\right): \mathrm{d}=23.4\left(\mathrm{CH}_{3}\right), 28.7\left(\mathrm{CH}_{2}\right), 56.8\left(\mathrm{CH}_{3}\right), 56.9\left(\mathrm{CH}_{3}\right)$, 
$59.2\left(\mathrm{CH}_{3}\right), 72.1\left(\mathrm{CH}_{2}\right), 73.4(\mathrm{CH}), 74.1(\mathrm{CH}), 74.7(\mathrm{CH}), 75.7(\mathrm{CH})$, 197.7 (C), $199.4 \mathrm{ppm}$ (C); IR (film): $\tilde{n}=2933,1716 \mathrm{~cm}^{-1}$; MS (EI): $\mathrm{m} / \mathrm{z}$ (\%): $260(<1)\left[M^{+}\right]$; HRMS (EI): $m / z$ : calcd for: $260.1260\left[M^{+}\right]$; found: 260.1261; elemental analysis calcd (\%) for $\mathrm{C}_{12} \mathrm{H}_{20} \mathrm{O}_{6}$ (260.28): $\mathrm{C}$ 55.37, $\mathrm{H}$ 7.74; found: C $55.52, \mathrm{H} 7.73$.

Photocyclization of 39: Method A: A deoxygenated solution of diketone 39 (41 mg, $0.156 \mathrm{mmol})$ in dry $\mathrm{C}_{6} \mathrm{D}_{6}(0.6 \mathrm{~mL})$, was placed in a resonance tube and irradiated with a daylight lamp at $30 \mathrm{8C}$ for $3 \mathrm{~h}$. The reaction was monitored by ${ }^{1} \mathrm{H}$ NMR spectroscopy, complete transformation into a mixture of the enol intermediate and the cyclized product 46 was observed. The mixture was heated at $458 \mathrm{C}$ in the dark for $1 \mathrm{~h}$, concentrated under reduced pressureand the residue purified by Chromatotron chromatography $\left(\mathrm{Al}_{2} \mathrm{O}_{3}\right.$, hexanes/EtOAc $\left.9: 1 ! 7: 3\right)$ to give $46(23 \mathrm{mg}$, $0.089 \mathrm{mmol}, 57 \%$ ) as a colorless oil.

Method B: A deoxygenated solution of diketone 39 (34 mg, $0.131 \mathrm{mmol}$ ) in dry $\mathrm{C}_{6} \mathrm{D}_{6}(0.6 \mathrm{~mL})$, was placed in a resonance tube and irradiated with a daylight lamp at $45 \mathrm{8C}$ for $7 \mathrm{~h}$. The reaction was monitored by ${ }^{1} \mathrm{H} \mathrm{NMR}$ spectroscopy, complete transformation into the cyclized product 46 was observed. The mixture was concentrated under reduced pressure and the residue purified by Chromatotron chromatography $\left(\mathrm{Al}_{2} \mathrm{O}_{3}\right.$, hexanes/ Et OAc $9: 1 ! 7: 3$ as eluents) to give $(2 S, 3 \mathrm{a} S, 4 R, 6 S, 6 \mathrm{a} R)-2$-hydroxy-4,6-dimethoxy-6a-(methoxymethyl)-2-methyltetrahydro- $2 H$-cyclopenta[b]furan-3(3a $\mathrm{H}$ )-one (46) (20 mg, $0.077 \mathrm{mmol}, 59 \%)$ as a colorless oil. [a] = D $+139 \mathrm{~cm}^{3} \mathrm{~g}^{-1} \mathrm{dm}^{-1}\left(c=1.01\right.$ in $\left.\mathrm{CHCl}_{3}\right) ;{ }^{1} \mathrm{H} \mathrm{N} \mathrm{MR}\left(500 \mathrm{M} \mathrm{Hz}, \mathrm{CDCl}_{3}\right): \mathrm{d}=$ $1.35(\mathrm{~d}, J=0.95 \mathrm{~Hz}, 3 \mathrm{H}, \mathrm{OH}$ coupling), 1.95 (ddd, $J=14.8,6.0,5.4 \mathrm{~Hz}$, $1 \mathrm{H}$ ), 2.13 (dddd, $J=14.5,3.2,3.2,1.3 \mathrm{~Hz}, 1 \mathrm{H}$ ), 3.13 (dd, $J=1.3,1.3 \mathrm{~Hz}$, $1 \mathrm{H}), 3.35(\mathrm{~s}, 3 \mathrm{H}), 3.37(\mathrm{~s}, 3 \mathrm{H}), 3.48(\mathrm{~s}, 3 \mathrm{H}), 3.75(\mathrm{~d}, J=10.1 \mathrm{~Hz}, 1 \mathrm{H})$ 3.76 (dd, $J=5.0,3.4 \mathrm{~Hz}, 1 \mathrm{H}$ ), 3.78 (d, $J=10.4 \mathrm{~Hz}, 1 \mathrm{H}$ ), 3.79 (ddd, $J=5.7$, 2.5, $2.5 \mathrm{~Hz}, 1 \mathrm{H}), 5.35 \mathrm{ppm}(\mathrm{d}, J=0.9 \mathrm{~Hz}, 1 \mathrm{H}, \mathrm{OH}) ;{ }^{1} \mathrm{H} \mathrm{N} \mathrm{MR}(500 \mathrm{MHz}$, $\mathrm{C}_{6} \mathrm{D}_{6}$ ): $\mathrm{d}=1.60(\mathrm{~s}, 3 \mathrm{H}$ ), 1.72 (ddd, $J=14.2,5.7,5.7 \mathrm{~Hz}, 1 \mathrm{H}$ ), 1.89 (ddd, $J=14.2,4.7,4.7 \mathrm{~Hz}, 1 \mathrm{H}), 2.70(\mathrm{~s}, 3 \mathrm{H}), 2.98(\mathrm{~s}, 3 \mathrm{H}), 3.13(\mathrm{~s}, 3 \mathrm{H}), 3.18(\mathrm{~d}$, $J=2.8 \mathrm{~Hz}, 1 \mathrm{H}), 3.507(\mathrm{dd}, J=5.0,5.0 \mathrm{~Hz}, 1 \mathrm{H}), 3.513(\mathrm{~d}, J=10.4 \mathrm{~Hz}, 1 \mathrm{H}$ ), $3.54(\mathrm{~d}, J=10.4 \mathrm{~Hz}, 1 \mathrm{H}), 3.66$ (ddd, $J=6.3,3.8,2.8 \mathrm{~Hz}, 1 \mathrm{H}), \quad 5.47 \mathrm{ppm}$ (br s, $1 \mathrm{H}) ;{ }^{13} \mathrm{C} \mathrm{NMR}\left(100.6 \mathrm{MHz}, \mathrm{CDCl}_{3}\right): \mathrm{d}=21.1\left(\mathrm{CH}_{3}\right), 35.4\left(\mathrm{CH}_{2}\right)$ $55.8(\mathrm{CH}), 57.3\left(\mathrm{CH}_{3}\right), 57.8\left(\mathrm{CH}_{3}\right), 59.5\left(\mathrm{CH}_{3}\right), 73.9\left(\mathrm{CH}_{2}\right), 84.5(\mathrm{CH})$, $87.5(\mathrm{CH}), 91.2(\mathrm{C}), 98.7(\mathrm{C}), 210.1 \mathrm{ppm}(\mathrm{C}) ;{ }^{13} \mathrm{C} \mathrm{NMR}(125.7 \mathrm{MHz}$, $\left.\mathrm{C}_{6} \mathrm{D}_{6}\right): \mathrm{d}=21.6\left(\mathrm{CH}_{3}\right), 35.8\left(\mathrm{CH}_{2}\right), 55.6(\mathrm{CH}), 56.8\left(\mathrm{CH}_{3}\right), 57.1\left(\mathrm{CH}_{3}\right)$ $58.7\left(\mathrm{CH}_{3}\right), 74.3\left(\mathrm{CH}_{2}\right), 83.4(\mathrm{CH}), 87.6(\mathrm{CH}), 91.6(\mathrm{C}), 99.6(\mathrm{C})$, $209.6 \mathrm{ppm}(\mathrm{C}) ; \operatorname{IR}$ (film): $\tilde{n}=3348,2936,1760 \mathrm{~cm}^{-1}$; MS $\left(\mathrm{ESI}^{+}\right): \mathrm{m} / \mathrm{z}$ (\%): $283(100)\left[M^{+}+\mathrm{Na}\right]$; HRMS $\left(\mathrm{ESI}^{+}\right): \mathrm{m} / \mathrm{z}$ calcd for $\mathrm{C}_{12} \mathrm{H}_{20} \mathrm{NaO}_{6}$ : 283.1158 $\left[\mathrm{M}^{+}+\mathrm{Na}\right]$; found: 283.1147 ; elemental analysis calcd $(\%)$ for $\mathrm{C}_{12} \mathrm{H}_{20} \mathrm{O}_{6}$ (260.28):C 55.37, $\mathrm{H}$ 7.74; found: C 55.38, $\mathrm{H} \quad 7.74$.

4,8-Anhydro-7,9-bis-O-(tert-butyldiphenylsilyl)-1,6-dideoxy-5-O-methyld-altro-nono-2,3-diulose (40): Prepared from 34 (101 mg, $0.15 \mathrm{mmol}$ ) following the general procedure (Method A) using dimethyl sulfide (22 $\mathrm{mL}$, $0.3 \mathrm{mmol}$ ). The mixture wasstirred at room temperature in the dark for $5 \mathrm{~h}$ and concentrated under reduced pressure. The residue was purified by rapid silica gel column chromatography (hexanes/EtOAc 95:5!9:1) to give $40(81.4 \mathrm{mg}, 0.11 \mathrm{mmol}, 77 \%)$ as a yellow oil: $[\mathrm{a}]_{\mathrm{D}}=+$ $57.9 \mathrm{~cm}^{3} \mathrm{~g}^{-1} \mathrm{dm}^{-1}\left(c=0.95\right.$ in $\left.\mathrm{CHCl}_{3}\right) ;{ }^{1} \mathrm{H} \mathrm{NMR}$ (500 MHz, $\left.\mathrm{CDCl}_{3}\right): \mathrm{d}=$ 0.98 (s, 9 H), 1.01 (s, 9 H), 1.56 (ddd, $J=12.6,9.3,9.0 \mathrm{~Hz}, 1 \mathrm{H}$ ), 1.91 (ddd, $J$ $=12.5,4.7,4.7 \mathrm{~Hz}, 1 \mathrm{H}), 2.25(\mathrm{~s}, 3 \mathrm{H}), 3.10(\mathrm{~s}, 3 \mathrm{H}), 3.44(\mathrm{ddd}, J=10.4,6.9$, $4.6 \mathrm{~Hz}, 1 \mathrm{H}$ ), 3.75 (dd, $J=11.2,4.9 \mathrm{~Hz}, 1 \mathrm{H}$ ), 3.82 (ddd, $J=10.1,9.8,4.5 \mathrm{~Hz}$, $1 \mathrm{H}$ ), 3.96 (dd, $J=11.4,2.2 \mathrm{~Hz}, 1 \mathrm{H}$ ), 4.21 (ddd, $J=10.7,4.9,2.9 \mathrm{~Hz}, 1 \mathrm{H}$ ), $5.30(\mathrm{~d}, J=6.7 \mathrm{~Hz}, 1 \mathrm{H}), 7.27-7.42(\mathrm{~m}, 12 \mathrm{H}), 7.61-7.64 \mathrm{ppm}(\mathrm{m}, 8 \mathrm{H}) ;{ }^{13} \mathrm{C}$ NMR (125.7 MHz, CDCl $\left.)_{3}\right): d=19.2(\mathrm{C}), 19.3(\mathrm{C}), 23.5\left(\mathrm{CH}_{3}\right), 26.8(6 \times$ $\left.\mathrm{CH}_{3}\right), 34.0\left(\mathrm{CH}_{2}\right), 56.9\left(\mathrm{CH}_{3}\right), 63.9\left(\mathrm{CH}_{2}\right), 66.3(\mathrm{CH}), 72.9(\mathrm{CH}), 75.8(\mathrm{CH})$, $78.0(\mathrm{CH}), 127.5(4 \times \mathrm{CH}), 127.6(2 \times \mathrm{CH}), 127.7(2 \times \mathrm{CH}), 129.42(\mathrm{CH})$, $129.44(\mathrm{CH}), 129.7(\mathrm{CH}), 129.8(\mathrm{CH}), 133.3(\mathrm{C}), 133.6(\mathrm{C}), 133.9(\mathrm{C}), 134.1$ (C), $135.7(2 \times \mathrm{CH}), 135.76(2 \times \mathrm{CH}), 135.78(2 \times \mathrm{CH}), 135.82(2 \times \mathrm{CH})$, 197.9 (C), $200.3 \mathrm{ppm}(\mathrm{C})$; IR $\left(\mathrm{CHCl}_{3}, 0.2 \mathrm{~mm}\right): \tilde{\mathrm{n}}=2933,1714 \mathrm{~cm}^{-1}$; MS (EI): $m / z(\%): 708(<1)\left[M^{+}\right], 651$ (18), 135 (100); HRMS (EI): $m / z$ calcd for $\mathrm{C}_{42} \mathrm{H}_{52} \mathrm{O}_{6} \mathrm{Si}_{2}$ : $708.3302\left[M^{+}\right]$; found: 708.3286; elemental analysis calcd (\%) for $\mathrm{C}_{42} \mathrm{H}_{52} \mathrm{O}_{6} \mathrm{Si}_{2}$ (709.03): C 71.15, H 7.39; found: C 71.48, H 7.00. (3Z,5R,7S)-7,9-Bis[(tert-butyldiphenylsilyl)oxy]-3-hydroxy-5-methoxy-3nonene-2,8-dione (50): A deoxygenated solution of diketone $40(56 \mathrm{mg}$, $0.079 \mathrm{mmol})$ in purified $\mathrm{CDCl}_{3}(0.6 \mathrm{~mL})$, was placed in a resonance tube and irradiated with a daylight lamp at 15-20 8C. The reaction was monitored by ${ }^{1} \mathrm{H}$ NMR spectroscopy, complete consumption of starting material 5 was observed after $12 \mathrm{~h}$, leading to exclusive formation of photoen- ol 50 . The solution could be concentrated at low temperature under re- duced pressure but 50 was not stable enough to withstand chromato- graphic purification (silica gel or alumina). Notwithstanding, the crude residue (colorless oil) was pure enough to allow the complete analytical and spectroscopic characterization. [a $]_{\mathrm{D}}=+32.3 \mathrm{~cm}^{3} \mathrm{~g}^{-1} \mathrm{dm}^{-1}(c=1.28 \mathrm{in}$ $\left.\mathrm{CHCl}_{3}\right) ;{ }^{1} \mathrm{H} \mathrm{NMR}\left(500 \mathrm{MHz}, \mathrm{CDCl}_{3}, 20 \mathrm{8C}\right): \mathrm{d}=0.94(\mathrm{~s}, 9 \mathrm{H}), 0.95(\mathrm{~s}, 9 \mathrm{H})$, 1.63 (ddd, $J=14.3,6.6,2.8 \mathrm{~Hz}, 1 \mathrm{H}$ ), 2.10 (ddd, $J=14.4,10.6,2.2 \mathrm{~Hz}$,

$1 \mathrm{H}$ ), $2.24(\mathrm{~s}, 3 \mathrm{H}), 3.03(\mathrm{~s}, 3 \mathrm{H}), 4.20(\mathrm{~d}, J=17.7 \mathrm{~Hz}, 1 \mathrm{H}), 4.38$ (dd, $J=6.3$, $2.2 \mathrm{~Hz}, 1 \mathrm{H}$ ), 4.52 (ddd, $J=10.8,8.0,2.7 \mathrm{~Hz}, 1 \mathrm{H}), 4.53$ (d, $J=17.9 \mathrm{~Hz}$, $1 \mathrm{H}$ ), 5.34 (dd, $J=8.2,1.3 \mathrm{~Hz}, 1 \mathrm{H}$ ), 6.49 (d, $J=1.3 \mathrm{~Hz}, 1 \mathrm{H}), 7.16-7.34$ (m, $10 \mathrm{H}), 7.43-7.57 \mathrm{ppm}(\mathrm{m}, 10 \mathrm{H}) ;{ }^{13} \mathrm{C} \mathrm{NMR}\left(125.7 \mathrm{MHz}, \mathrm{CDCl}_{3}, 208 \mathrm{C}\right): \mathrm{d}=$ $19.18(\mathrm{C}), 19.21(\mathrm{C}), 23.1\left(\mathrm{CH}_{3}\right), 26.7\left(3 \times \mathrm{CH}_{3}\right), 26.9\left(3 \times \mathrm{CH}_{3}\right), 34.5$ $\left(\mathrm{CH}_{2}\right), 56.7\left(\mathrm{CH}_{3}\right), 68.3\left(\mathrm{CH}_{2}\right), 71.1(\mathrm{CH}), 74.6(\mathrm{CH}), 115.6(\mathrm{CH}), 127.6$ $(2 \times \mathrm{CH}), 127.68(4 \times \mathrm{CH}), 127.71(2 \times \mathrm{CH}), 129.67(\mathrm{CH}), 129.69(\mathrm{CH})$, $129.86(\mathrm{CH}), 129.88(\mathrm{CH}), 133.6(\mathrm{C}), 133.8(\mathrm{C}), 133.06(\mathrm{C}), 134.12(\mathrm{C})$, $135.5(4 \times \mathrm{CH}), 135.7(2 \times \mathrm{CH}), 135.8(2 \times \mathrm{CH}), 148.4(\mathrm{C}), 194.9(\mathrm{C})$, $208.6 \mathrm{ppm}$ (C); IR $\left(\mathrm{CHCl}_{3}, 0.2 \mathrm{~mm}\right): \tilde{n}=3441,2246,1733,1684$ $1661 \mathrm{~cm}^{-1}$; UV/Vis $\left(\mathrm{CH}_{2} \mathrm{Cl}_{2}\right): \mathrm{I}_{\max }(\mathrm{e})=271$ (sh) (3055), 264 (3841), 261 (3877), 255 (sh) (3441), 227 (5628 mol-1 $\mathrm{m}^{3} \mathrm{~cm}^{-1}$ ); MS (EI): $\mathrm{m} / \mathrm{z}(\%): 665$ <1) $\left[\mathrm{M}^{+}\right.$-Ac], 651(4), 135(100); HRMS $\left(\mathrm{ESI}^{+}\right): \mathrm{m} / \mathrm{zcalcd}$ for $\mathrm{C}_{42} \mathrm{H}_{52} \mathrm{NaO}_{6} \mathrm{Si}_{2}: 731.3200[M+\mathrm{Na}]$; found: 731.3198 ; elemental analysis calcd (\%) for $\mathrm{C}_{42} \mathrm{H}_{52} \mathrm{O}_{6} \mathrm{Si}_{2}$ (709.03): $\mathrm{C}$ 71.15, $\mathrm{H}$ 7.39; found: $\mathrm{C} 71.02, \mathrm{H}$ 7.40

Kinetic studies: The unlabeled photoenol 50 (9.4 mg, $0.013 \mathrm{mmol})$ was dissolved in $\left[\mathrm{D}_{6}\right]$ benzene $(0.6 \mathrm{~mL})$, transferred to an NMR tube and immediately placed into the preheated probe $(40 \mathrm{8C})$ of a $500 \mathrm{MHz} \mathrm{NMR}$ spectrometer to minimize spontaneous cyclization of 50. The integral of vinyl proton at $5.10 \mathrm{ppm}(1 \mathrm{H}, \mathrm{dd}, J=8.2,1.3 \mathrm{~Hz})$ were monitored over $14 \mathrm{~h}$ and data were collected every $30 \mathrm{~min}$. The same procedure was car- ried out for the deuterated photoenol $\left[\mathrm{D}_{1}\right] 50(11.5 \mathrm{mg}, 0.016 \mathrm{mmol})$ dis- solved in $\left[D_{6}\right]$ benzene $(0.6 \mathrm{~mL})$, prepared by hydroxyl proton exchange with $\mathrm{D}_{2} \mathrm{O}$. The vinyl proton now appears at $5.11 \mathrm{ppm}(1 \mathrm{H}, \mathrm{d}, J=8.2 \mathrm{~Hz})$. Since $\left[\mathrm{D}_{1}\right] 50$ was not isotopically pure (contained $11 \%$ of unlabeled 50 ) the appropriate corrections were introduced during the concentration cal- culations. The kinetic isotope effect (KIE) value $\left(k_{\mathrm{H}} / k_{\mathrm{D}}=3.7\right)$ was com- puted by plotting the selected concentration data against time and fitting the data to a firstorder simulation to obtain the rate constants for the cyclization of both 50 $\left(k_{\mathrm{H}}=5.42 \times 10^{-5}, R^{2}=0.999\right)$ and $\left[\mathrm{D}_{1}\right] 50\left(k_{\mathrm{D}}=1.48 \times 10^{-5}, R^{2}=0.999\right)$.

Photocyclization of 40: A deoxygenated solution of diketone $40(19 \mathrm{mg}$, $0.027 \mathrm{mmol})$ in dry $\mathrm{C}_{6} \mathrm{D}_{6}(0.6 \mathrm{~mL})$, was placed in a resonance tube and irradiated with a daylight lamp at $258 \mathrm{C}$ for $7 \mathrm{~h}$. The reaction was moni- tored by ${ }^{1} \mathrm{H}$ NMR spectroscopy, complete transformation into the enol intermediate 50 was observed. The enol was heated at $40 \mathrm{8C}$ in the dark for $8 \mathrm{~h}$, leading to exclusive formation of carbocycle 47. The solution was concentrated under reduced pressure and the residue purified by Chromatotron chromatography on alumina (hexanes/EtOAc 9:1) to give $(2 S, 3 a S, 4 R, 6 S, 6 a R)-4$-(benzyloxy)-6-[(tert-butyldiphenylsilyl)oxy]-6a-

[(tert-butyldiphenylsilyl)oxy]methyl-2-hydroxy-2-methyltetrahydro- $2 \mathrm{H}$-cyclopenta[b]furan-3(3aH)-one (47) (14.8 mg, $0.021 \mathrm{mmol}, 78 \%)$ as a crystalline solid: M.p. $105.7-107.98 \mathrm{C}(\mathrm{MeOH}) ;[\mathrm{a}]_{\mathrm{D}}=+30.3 \mathrm{~cm}^{3} \mathrm{~g}^{-1} \mathrm{dm}^{-1}(\mathrm{c}=$ 1.56 in $\left.\mathrm{CHCl}_{3}\right)$; ${ }^{1} \mathrm{H} \mathrm{NMR}\left(500 \mathrm{MHz} \mathrm{CDCl}_{3}\right): \mathrm{d}=0.82(\mathrm{~s}, 9 \mathrm{H}), 1.08(\mathrm{~s}$, 9H), 1.36 (s, $3 \mathrm{H}$ ), 1.65 (dddd, $J=13.9,4.6,4.6,0.7 \mathrm{~Hz}, 1 \mathrm{H}$ ), 1.76 (ddd, $J=14.2,5.7,5.7 \mathrm{~Hz}, 1 \mathrm{H}), 3.18(\mathrm{dd}, J=2.6,0.7 \mathrm{~Hz}, 1 \mathrm{H}), 3.26(\mathrm{~s}, 3 \mathrm{H}), 3.63$ (ddd, $J=5.9,4.7,2.9 \mathrm{~Hz}, 1 \mathrm{H}), 4.00(\mathrm{~d}, J=11.7 \mathrm{~Hz}, 1 \mathrm{H}), 4.05(\mathrm{~d}, J=$ $11.7 \mathrm{~Hz}, 1 \mathrm{H}), 4.14(\mathrm{dd}, J=5.4,5.1 \mathrm{~Hz}, 1 \mathrm{H}), 5.38(\mathrm{~s}, 1 \mathrm{H}), 7.24-7.29(\mathrm{~m}$, $4 \mathrm{H}), 7.35-7.51(\mathrm{~m}, 12 \mathrm{H}), 7.64-7.67 \mathrm{ppm}(\mathrm{m}, 4 \mathrm{H}) ;{ }^{13} \mathrm{C} \mathrm{N} \mathrm{MR}(100.6 \mathrm{MHz}$, $\left.\mathrm{CDCl}_{3}\right): \mathrm{d}=19.1(\mathrm{C}), 19.2(\mathrm{C}), 20.7\left(\mathrm{CH}_{3}\right), 26.7\left(3 \times \mathrm{CH}_{3}\right), 26.9\left(3 \times \mathrm{CH}_{3}\right)$,

$40.0\left(\mathrm{CH}_{2}\right), 54.8(\mathrm{CH}), 56.8(\mathrm{CH})_{3} 66.8(\mathrm{CH})_{2} 79.6(\mathrm{CH}), 82.2(\mathrm{CH}), 93.3$ (C), $99.0(\mathrm{C}), 127.5(2 \times \mathrm{CH}), 127.6(2 \times \mathrm{CH}), 128.0(2 \times \mathrm{CH}), 128.1(2 \times$ $\mathrm{CH}), 129.8(2 \times \mathrm{CH}), 130.1(\mathrm{CH}), 130.3(\mathrm{CH}), 131.4(\mathrm{C}), 131.5(\mathrm{C}), 132.8$ (C), $133.5(\mathrm{C}), 135.6(2 \times \mathrm{CH}), 135.76(2 \times \mathrm{CH}), 135.81(2 \times \mathrm{CH}), 135.89$ $(2 \times \mathrm{CH}), 209.9 \mathrm{ppm}(\mathrm{C})$; IR $\left(\mathrm{CHCl}_{3}, 0.2 \mathrm{~mm}\right): \bar{n}=3672,3357,2932$, $1763 \mathrm{~cm}^{-1}$; MS (EI): $m / z(\%): 651(1)\left[M^{+}-t \mathrm{Bu}\right], 633(4), 135(100)$; HRMS $\left(\mathrm{ESI}^{+}\right)$: $m / z$ calcd for $\mathrm{C}_{42} \mathrm{H}_{52} \mathrm{NaO}_{6} \mathrm{Si}_{2}: 731.3200\left[M^{+}+\mathrm{Na}\right]$; found: 
731.3213; elemental analysis calcd (\%) for $\mathrm{C}_{42} \mathrm{H}_{52} \mathrm{O}_{6} \mathrm{Si}_{2}$ (709.03): $\mathrm{C} 71.15, \mathrm{H}$ 7.39; found: C 71.21, H 7.40.

4,8-Anhydro-6,7-dideoxy-5,9-di-O-methyl-d-arabino-nono-2,3-diulose (41): Prepared from 35 (25.3 $\mathrm{mg}, 0.13 \mathrm{mmol})$ following the general procedure (Method A) using dimethyl sulfide ( $40 \mathrm{~mL}, 0.54 \mathrm{mmol}$ ). The mixture was stirred at room temperature in the dark for $2 \mathrm{~h}$ and concentrated under reduced pressure. The residue was purified by rapid silica gel column chromatography (hexanes/EtOAc 8:2) to give 41 (20.4 mg, $0.09 \mathrm{mmol}, 69 \%)$ as a yellow oil. $[\mathrm{a}]_{\mathrm{D}}=+116 \mathrm{~cm}^{3} \mathrm{~g}^{-1} \mathrm{dm}^{-1}(\mathrm{c}=2.57 \mathrm{in}$ $\left.\mathrm{CHCl}_{3}\right) ;{ }^{1} \mathrm{H} \mathrm{NMR}\left(500 \mathrm{MHz}, \mathrm{CDCl}_{3}\right): \mathrm{d}=1.48(\mathrm{~m}, 1 \mathrm{H}), 1.65(\mathrm{~m}, 1 \mathrm{H})$, $1.75(\mathrm{~m}, 1 \mathrm{H}), 1.97(\mathrm{~m}, 1 \mathrm{H}), 2.27(\mathrm{~s}, 3 \mathrm{H}), 3.27(\mathrm{~s}, 3 \mathrm{H}), 3.38(\mathrm{~s}, 3 \mathrm{H}), 3.39$ 3.43 (m, $2 \mathrm{H}$ ), 3.74 (ddd, $J=9.2,6.3,4.0 \mathrm{~Hz}, 1 \mathrm{H}), 4.44(\mathrm{~m}, 1 \mathrm{H}), 5.43 \mathrm{ppm}$ (d, $J=6.3 \mathrm{~Hz}, 1 \mathrm{H}) ;{ }^{13} \mathrm{C} \mathrm{NMR}\left(125.7 \mathrm{MHz}, \mathrm{CDCl}_{3}\right): \mathrm{d}=23.50\left(\mathrm{CH}_{2}\right), 23.54$ $\left(\mathrm{CH}_{2}\right), 24.1\left(\mathrm{CH}_{2}\right), 56.9\left(\mathrm{CH}_{3}\right), 59.2\left(\mathrm{CH}_{3}\right), 70.6(\mathrm{CH}), 74.1(\mathrm{CH}), 75.0$ $\left(\mathrm{CH}_{2}\right), 76.3(\mathrm{CH}), 197.8(\mathrm{C}), 200.8 \mathrm{ppm}(\mathrm{C})$; IR $\left(\mathrm{CHCl}_{3}, 0.2 \mathrm{~mm}\right): \tilde{\mathrm{n}^{2}}=$ 2934, 1771, 1714, $1099 \mathrm{~cm}^{-1}$; MS (ESI $\left.{ }^{+}\right): m / z(\%): 253(100)\left[M^{+}+\mathrm{Na}\right]$, 229(3); HRMS (ESI ${ }^{+}$): $m / z$ calcd for C $\mathrm{H}_{1 \mathrm{Na}} \mathrm{Q}: 253.1052\left[M^{+}+\mathrm{Na}\right]$; found: 253.1042; elemental analysis calcd (\%) for $\mathrm{C}_{11} \mathrm{H}_{18} \mathrm{O}_{5}$ (230.26): $\mathrm{C}$ 57.38, H 7.88; found: C 57.30, H 7.85.

(3E,5R)-3-Hydroxy-5,9-dimethoxy-3-nonene-2,8-dione (51): A deoxygenated solution of diketone $41(37.0 \mathrm{mg}, 0.16 \mathrm{mmol})$ in purified $\mathrm{CDCl}_{3}(0.3$ $\mathrm{mL}$ ), was placed in a resonance tube and irradiated with a daylight lamp at 30-33 8C. The reaction was monitored by ${ }^{1} \mathrm{H}$ N M R spectroscopy; complete consumption of starting material was observed after $4.5 \mathrm{~h}$, lead- ing to exclusive formation of intermediate photoenol 51 . The solution could be concentrated at low temperature under reduced pressure but 51 was not stable enough to withstand chromatographic purification (silica gel or alumina). Notwithstanding, the crude residue (32.5 mg, $0.14 \mathrm{mmol}, 88 \%$ ) was pure enough to allow the complete analytical and spectroscop- ic characterization.

Compound 51: colorless oil. [a] $]_{\mathrm{D}}=+19 \mathrm{~cm}^{3} \mathrm{~g}^{-1} \mathrm{dm}^{-1}\left(c=0.32\right.$ in $\left.\mathrm{CHCl}_{3}\right)$; ${ }^{1} \mathrm{H} \mathrm{NMR}\left(500 \mathrm{MHz}, \mathrm{CDCl}_{3}\right): \mathrm{d}=1.89(\mathrm{~m}, 1 \mathrm{H}), 1.96(\mathrm{~m}, 1 \mathrm{H}), 2.38(\mathrm{~s}$, $3 \mathrm{H}$ ), 2.50 (ddd, $J=17.4,8.2,6.1 \mathrm{~Hz}, 1 \mathrm{H}$ ), 2.59 (ddd, $J=17.4,8.2,6.6 \mathrm{~Hz}$, $1 \mathrm{H}), 3.30(\mathrm{~s}, 3 \mathrm{H}), 3.41(\mathrm{~s}, 3 \mathrm{H}), 4.01(\mathrm{~s}, 2 \mathrm{H}), 4.27$ (ddd, $J=8.3,7.4$, $5.6 \mathrm{~Hz}, 1 \mathrm{H}$ ), 5.48 (dd, $J=8.3,1.2 \mathrm{~Hz}, 1 \mathrm{H}), 6.61 \mathrm{ppm}(\mathrm{d}, J=1.2 \mathrm{~Hz}, 1 \mathrm{H})$; ${ }^{13} \mathrm{C} \mathrm{N}$ MR $\left(125.7 \mathrm{MHz}, \mathrm{CDCl}_{3}\right): \mathrm{d}=23.1\left(\mathrm{CH}_{3}\right), 28.2\left(\mathrm{CH}_{2}\right), 34.6\left(\mathrm{CH}_{2}\right)$, $56.9(\mathrm{CH}), 59.3(\mathrm{CH}),{ }_{3} 75.1(\mathrm{CH}), 77.6(\mathrm{CH}), 115.5(\mathrm{CH}), 148.8(\mathrm{C})$ 194.8 (C), $208.1 \mathrm{ppm}(\mathrm{C})$; IR ( $\mathrm{CHCl}, 0.2 \mathrm{~mm}): \tilde{n}=3435,2935,2252$, 1731, 1682, 1663, $1350 \mathrm{~cm}^{-1}$; UV/Vis $\left(\mathrm{CH}_{2} \mathrm{Cl}_{2}\right): I_{\max }(\mathrm{e})=260 \mathrm{~nm}$ (8003 $\mathrm{mol}^{-1} \mathrm{~m}^{3} \mathrm{~cm}^{-1}$ ); MS (EI): $m / z(\%): 230(1)\left[M^{+}\right], 213(1), 199(1)$, 198(12); HRMS (EI): $m / z$ : calcd for $\mathrm{C}_{11} \mathrm{H}_{18} \mathrm{O}_{5}: 230.1154$ [ $M^{+}$]; found: 230.1153; elemental analysis calcd (\%) for $\mathrm{C}_{11} \mathrm{H}_{18} \mathrm{O}_{5}(230.26): \mathrm{C} 57.38, \mathrm{H}$ 7.88; found: C $57.58, \mathrm{H} 7.91$.

Photocyclization of 41: A deoxygenated solution of diketone 41 (51.2 mg, $0.23 \mathrm{mmol})$ in dry C $\mathrm{D}_{6}(0.4 \mathrm{~mL})$, was placed in a resonance tube and irradiated with a daylight lamp at $30 \mathrm{8C}$ for $4.5 \mathrm{~h}$. The reaction was monitored by ${ }^{1} \mathrm{H}$ NMR spectroscopy, complete transformation into the intermediate photoenol 51 was observed. The mixture was heated at $608 \mathrm{C}$ in the dark for $7 \mathrm{~h}$, concentrated under reduced pressure and the residue purified by Chromatotron chromatography $\left(\mathrm{Al}_{2} \mathrm{O}_{3}\right.$, hexanes/EtOAc 8:2) to give $(2 S, 3 \mathrm{a} S, 4 R, 6 \mathrm{a} R)$-2-hydroxy-4-methoxy-6a-(methoxymethyl)-2-

methyltetrahydro- $2 H$-cyclopenta $[b]$ furan- $3(3 a H)$-one (48) $\quad(26.8 \mathrm{mg}$, $0.12 \mathrm{mmol}, 52 \%)$ as a colorless oil: [a] $=_{\mathrm{D}}+101 \mathrm{~cm}^{3} \mathrm{~g}^{-1} \mathrm{dm}^{-1}(c=0.34 \mathrm{in}$ $\left.\mathrm{CHCl}_{3}\right) ;{ }^{1} \mathrm{H} \mathrm{NMR}(500 \mathrm{M} \mathrm{Hz}, \mathrm{CDCl}$ ): $\mathrm{d}=1.37(\mathrm{br} \mathrm{d}, J=1.4 \mathrm{~Hz}, 3 \mathrm{H}), 1.52$ (dddd, $J=13.5,12.2,8.0,4.5 \mathrm{~Hz}, 1 \mathrm{H}$ ), 1.87 (m, $2 \mathrm{H}$ ), 1.94 (dddd, $J=13.5$, $5.8,1.6,1.6 \mathrm{~Hz}, 1 \mathrm{H}), 3.05(\mathrm{~d}, J=1.9 \mathrm{~Hz}, 1 \mathrm{H}), 3.32(\mathrm{~s}, 3 \mathrm{H}), \quad 3.38(\mathrm{~d}, J=$ $9.8 \mathrm{~Hz}, 1 \mathrm{H}), 3.48(\mathrm{~s}, 3 \mathrm{H}), 3.74(\mathrm{~d}, J=9.8 \mathrm{~Hz}, 1 \mathrm{H}), 3.86 \mathrm{ppm}(\mathrm{d}, J=$ $4.2 \mathrm{~Hz}, 1 \mathrm{H}) ;{ }^{1} \mathrm{H}$ NMR $\left(500 \mathrm{MHz}, \mathrm{C}_{6} \mathrm{D}_{6}\right): \mathrm{d}=1.26-1.35(\mathrm{~m}, 1 \mathrm{H}), 1.61(\mathrm{~d}$ $J=0.95 \mathrm{~Hz}, 3 \mathrm{H}), 1.58-1.69(\mathrm{~m}, 3 \mathrm{H}), 2.68(\mathrm{~s}, 3 \mathrm{H}), 2.83(\mathrm{~d}, J=9.8 \mathrm{~Hz}$, $1 \mathrm{H}), 2.94(\mathrm{~s}, 3 \mathrm{H}), 3.04(\mathrm{br} \mathrm{s}, 1 \mathrm{H}), 3.24$ (d, $J=9.5 \mathrm{~Hz}, 1 \mathrm{H}), 3.80$ (d, $J=$ $4.1 \mathrm{~Hz}, 1 \mathrm{H}), 5.40 \mathrm{ppm}(\mathrm{d}, J=1.3 \mathrm{~Hz}, 1 \mathrm{H}, \mathrm{OH}) ;{ }^{13} \mathrm{C} \mathrm{N} \mathrm{MR}(100.6 \mathrm{MHz}$, $\left.\mathrm{CDCl}_{3}\right): \mathrm{d}=20.4\left(\mathrm{CH}_{3}\right), 29.9\left(\mathrm{CH}_{2}\right), 33.9\left(\mathrm{CH}_{2}\right), 55.4(\mathrm{CH}), \quad 56.4\left(\mathrm{CH}_{3}\right)$, $59.2\left(\mathrm{CH}_{3}\right), 77.1\left(\mathrm{CH}_{2}\right), 85.7(\mathrm{CH}), 90.1(\mathrm{C}), 98.8(\mathrm{C}), 209.5 \mathrm{ppm}(\mathrm{C})$; IR $\left(\mathrm{CHCl}_{3}, 0.2 \mathrm{~mm}\right): \tilde{\mathrm{n}^{-}}=3364,2936,1762,1094 \mathrm{~cm}^{-1}$; $\mathrm{MS}\left(\mathrm{ESI}^{+}\right): \mathrm{m} / z(\%):$ $285(28)\left[M^{+}+\mathrm{MeOH}+\mathrm{Na}\right], 253(100)\left[M^{+}+\mathrm{Na}\right] ; H \mathrm{HMS}\left(\mathrm{ESI}^{+}\right): \mathrm{m} / \mathrm{z}$ calcd for $\mathrm{C}_{11} \mathrm{H}_{18} \mathrm{NaO}_{5}$ : $253.1052\left[M^{+}+\mathrm{Na}\right]$; found: 253.1047; elemental analysis calcd (\%) for $\mathrm{C}_{11} \mathrm{H}_{18} \mathrm{O}_{5}$ (230.25): $\mathrm{C} 57.38, \mathrm{H}$ 7.88; found: $\mathrm{C} 57.43$, H 7.87.
4,8-Anhydro-9-(tert-butyldiphenylsilyl)-1,6,7-trideoxy-5-O-methyl-d-arabino-nono-2,3-diulose (42): Prepared from $36(36.7 \mathrm{mg}, 0.09 \mathrm{mmol}$ ) following the general procedure (Method B) using $\mathrm{NaIO}_{4} \quad(56 \mathrm{mg}$, $0.26 \mathrm{mmol})$ and $\mathrm{RuO}_{2} \times \mathrm{H}_{2} \mathrm{O}(0.25 \mathrm{mg})$. The reaction mixture was stirred at room temperature in the dark for $30 \mathrm{~min}$. The residue was purified by rapid silica gel column chromatography (EtOAc) to give $42(28.1 \mathrm{mg}$, 0.06 mmol, $71 \%)$ as a yellow oil: $[\mathrm{a}]_{\mathrm{D}}=+46 \mathrm{~cm}^{3} \mathrm{~g}^{-1} \mathrm{dm}^{-1}(c=1.53 \mathrm{in}$ $\left.\mathrm{CHCl}_{3}\right) ;{ }^{1} \mathrm{H} \mathrm{NMR}\left(500 \mathrm{MHz}, \mathrm{CDCl}_{3}\right): \mathrm{d}=1.06(\mathrm{~s}, 9 \mathrm{H}), 1.51(\mathrm{~m}, 1 \mathrm{H}), 1.66$ $(\mathrm{m}, 1 \mathrm{H}), 1.88(\mathrm{~m}, 1 \mathrm{H}), 1.96(\mathrm{~m}, 1 \mathrm{H}), 2.29(\mathrm{~s}, 3 \mathrm{H}), 3.27(\mathrm{~s}, 3 \mathrm{H}), 3.66(\mathrm{dd}, J=$ $10.5,5.7 \mathrm{~Hz}, 1 \mathrm{H}$ ), 3.72 (ddd, $J=5.8,4.7,4.1 \mathrm{~Hz}, 1 \mathrm{H}$ ), 3.74 (dd, $J=10.5,4.9$ $\mathrm{Hz}, 1 \mathrm{H}), 4.33(\mathrm{~m}, 1 \mathrm{H}), 5.32$ (d, $J=5.8 \mathrm{~Hz}, 1 \mathrm{H}), 7.40(\mathrm{~m}, 6 \mathrm{H}), 7.67 \mathrm{ppm}$ $(\mathrm{m}, 4 \mathrm{H}) ;{ }^{13} \mathrm{C}$ NMR $\left(125.7 \mathrm{MHz}, \mathrm{CDCl}_{3}\right): \mathrm{d}=19.2(\mathrm{C}), 23.2\left(\mathrm{CH}_{2}\right), 23.6$ $\left(\mathrm{CH}_{2}\right), 23.7\left(\mathrm{CH}_{3}\right), 26.8\left(3 \times \mathrm{CH}_{3}\right), 56.8\left(\mathrm{CH}_{3}\right), 65.8\left(\mathrm{CH}_{2}\right), 72.5(\mathrm{CH}), 74.0$ $(\mathrm{CH}), 76.2(\mathrm{CH}), 127.6(4 \times \mathrm{CH}), 129.6(2 \times \mathrm{CH}), 133.46(\mathrm{C}), 133.49$ (C), $135.6(4 \times \mathrm{CH}), 198.1(\mathrm{C}), 200.6 \mathrm{ppm}(\mathrm{C})$; IR $\left(\mathrm{CHCl}_{3}\right.$,

$0.2 \mathrm{~mm}): \tilde{\mathrm{n}^{2}} 2936,1714 \mathrm{~cm}^{-1}$; MS (EI): $\mathrm{m} / \mathrm{z}(\%): 397$ (58) [ $\left.M^{+}-t \mathrm{Bu}\right], 199$ (75); HRMS (EI): $m / z$ calcd for $\mathrm{C}_{22} \mathrm{H}_{25} \mathrm{O}_{5} \mathrm{Si}: 397.1471$ [ $M^{+}-t \mathrm{Bu}$ ]; found: 397.1466; elemental analysis calcd (\%) for $\mathrm{C}_{26} \mathrm{H}_{34} \mathrm{O}_{5} \mathrm{Si}(454.63): \mathrm{C} 68.69, \mathrm{H}$ 7.54; found: C 68.61, H 7.60.

(3Z,5R)-9-[(tert-Butyldiphenylsilyl)oxy]-3-hydroxy-5-methoxy-3-nonene2,8-dione (52): A solution of $42(49.7 \mathrm{mg}, 0.11 \mathrm{mmol})$ in purified $\mathrm{CDCl}_{3}$ $(0.3 \mathrm{~mL})$, was placed in a resonance tube and irradiated with a daylightlamp at 30-358C for $3 \mathrm{~h}$. The reaction was monitored by using ${ }^{1} \mathrm{H} \mathrm{N} \mathrm{MR}$ spectroscopy to give the photoenol $52(47.3 \mathrm{mg}, 0.1 \mathrm{mmol}, 96 \%)$ as a yellowish oil that could not withstand chromatographic purification (silica gel or alumina). Nevertheless, the crude residue was pure enough to allow the complete analytical and spectroscopic characterization. $[\mathrm{a}]_{\mathrm{D}}=$

$+4 \mathrm{~cm}^{3} \mathrm{~g}^{-1} \mathrm{dm}^{-1}\left(c=0.47\right.$ in $\left.\mathrm{CHCl}_{3}\right) ;{ }^{1} \mathrm{H} \mathrm{NMR} \quad\left(500 \mathrm{MHz}, \mathrm{CDCl}_{3}\right): \mathrm{d}=$ $1.10(\mathrm{~s}, 9 \mathrm{H}), 1.84(\mathrm{~m}, 1 \mathrm{H}), 1.96(\mathrm{~m}, 1 \mathrm{H}), 2.36(\mathrm{~s}, 3 \mathrm{H}), 2.58$ (ddd, $J=17.8$, $8.2,6.3 \mathrm{~Hz}, 1 \mathrm{H}$ ), 2.67 (ddd, $J=17.8,8.5,6.3 \mathrm{~Hz}, 1 \mathrm{H}$ ), 3.29 (s, $3 \mathrm{H}$ ), 4.19 (s, $2 \mathrm{H}$ ), 4.26 (ddd, $J=8.2,7.3,6.0 \mathrm{~Hz}, 1 \mathrm{H}$ ), 5.47 (dd, $J=8.2,1.1 \mathrm{~Hz}, 1 \mathrm{H}$ ), $6.60(\mathrm{~d}, J=1.1 \mathrm{~Hz}, 1 \mathrm{H}), 7.37-7.44(\mathrm{~m}, 6 \mathrm{H}), 7.64-7.67 \mathrm{ppm}(\mathrm{m}, 4 \mathrm{H})$; ${ }^{13} \mathrm{CNMR}\left(125.7 \mathrm{MHz}, \mathrm{CDCl}_{3}\right): \mathrm{d}=19.2(\mathrm{C}), 23.1\left(\mathrm{CH}_{3}\right), 26.7\left(3 \times \mathrm{CH}_{3}\right)$, $28.0\left(\mathrm{CH}_{2}\right), 34.3\left(\mathrm{CH}_{2}\right), 56.9\left(\mathrm{CH}_{3}\right), 69.7\left(\mathrm{CH}_{2}\right), 75.1(\mathrm{CH}), 115.5(\mathrm{CH})$, $127.8(4 \times \mathrm{CH}), 129.9(2 \times \mathrm{CH}), 132.7(2 \times \mathrm{C}), 135.5(4 \times \mathrm{CH}), 148.7(\mathrm{C})$, 194.8 (C), $209.6 \mathrm{ppm}$ (C); IR $\left(\mathrm{CHCl}_{3}, 0.2 \mathrm{~mm}\right): \tilde{n}=3435,2933,2249$, 1714, 1681, $1662 \mathrm{~cm}^{-1}$; UV/Vis $\left(\mathrm{CH}_{2} \mathrm{Cl}_{2}\right): I_{\max }(\mathrm{e})=272(\mathrm{sh})$ (2914), 264

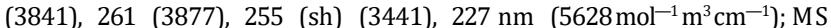
(EI): $m / z(\%): 437$ (5) [ $M^{+}-\mathrm{OH}$ ], 365 (38), 199(100); HRMS (EI): $m / z$ calcd for $\mathrm{C}_{26} \mathrm{H}_{33} \mathrm{O}_{4} \mathrm{Si}: 437.2148\left[M^{+}-\mathrm{OH}\right]$; found: 437.2140; elemental analysis calcd (\%) for $\mathrm{C}_{26} \mathrm{H}_{34} \mathrm{O}_{5} \mathrm{Si}$ (454.63): C 68.69, $\mathrm{H}$ 7.54; found: C 68.83, H 7.30.

Photocyclization of 42: A deoxygenated solution of diketone 42 (58.7 mg, $0.127 \mathrm{mmol})$ in dry $\mathrm{C}_{6} \mathrm{D}_{6}(0.3 \mathrm{~mL})$, was irradiated with a day- light lamp at 36-40 8C for $5 \mathrm{~h}$. The reaction was monitored by ${ }^{1} \mathrm{H}$ NMR spectroscopy, complete transformation into the enol intermediate 52 was observed. The enol was heated at $60 \mathrm{8C}$ in the dark for $2 \mathrm{~h}$, concentrated under reduced pressure and the residue purified by column chromatogra- phy $\left(\mathrm{Al}_{2} \mathrm{O}_{3}\right.$, hexanes/EtOAc 95:5) to give an inseparable mixture of hem- iketal diastereomers ( $35.9 \mathrm{mg}, 0.078 \mathrm{mmol}$, d.r. 10:1,61\%) (major

isomer: $\quad(2 S, 3 \mathrm{a} S, 4 R, 6 \mathrm{a} R)-6 \mathrm{a}$-[(tert-butyldiphenylsilyl)oxy]methyl-2-hydroxy-4-methoxy-2-methyltetrahydro- $2 H$-cyclopenta $[b]$ furan-3(3a $H)$-one (49)) as a colorless oil: $[\mathrm{a}]_{\mathrm{D}}=+62 \mathrm{~cm}^{3} \mathrm{~g}^{-1} \mathrm{dm}^{-1}\left(c=0.98\right.$ in $\left.\mathrm{CHCl}_{3}\right) ;{ }^{1} \mathrm{H}$ $\mathrm{NMR}\left(500 \mathrm{M} \mathrm{Hz} \mathrm{CDCl}_{3}\right): \mathrm{d}$ (major isomer) $=1.07(\mathrm{~s}, 9 \mathrm{H}), 1.45(\mathrm{~s}, 3 \mathrm{H})$, 1.52 (dddd, $J=12.9,12.9,6.9,4.1 \mathrm{~Hz}, 1 \mathrm{H}$ ), 1.68 (ddd, $J=12.9,12.9$, $6.6 \mathrm{~Hz}, 1 \mathrm{H}$ ), 1.78 (ddd, $J=13.2,6.9,0.0 \mathrm{~Hz}, 1 \mathrm{H}$ ), 1.86 (dddd, $J=13.5,6.4$, $1.3,1.3 \mathrm{~Hz}, 1 \mathrm{H}), 3.10(\mathrm{~d}, J=1.9 \mathrm{~Hz}, 1 \mathrm{H}), 3.22(\mathrm{~s}, 3 \mathrm{H}), 3.48$ (d, $J=$ $11.0 \mathrm{~Hz}, 1 \mathrm{H}$ ), 3.86 (br d, $J=4.1 \mathrm{~Hz}, 1 \mathrm{H}), 3.99(\mathrm{~d}, J=11.0 \mathrm{~Hz}, 1 \mathrm{H}), 5.35$ $(\mathrm{d}, J=0.9 \mathrm{~Hz}, 1 \mathrm{H}), 7.38-7.49(\mathrm{~m}, 6 \mathrm{H}), 7.66-7.69 \mathrm{ppm}(\mathrm{m}, 4 \mathrm{H}) ;{ }^{13} \mathrm{C} \mathrm{N} \mathrm{M} \mathrm{R}$ $\left(100.6 \mathrm{MHz} \mathrm{CDCl}_{3}\right.$;): $\mathrm{d}$ (major isomer) $=19.1(\mathrm{C}), 20.3\left(\mathrm{CH}_{3}\right), 26.8(3 \times$ $\left.\mathrm{CH}_{3}\right), 30.3\left(\mathrm{CH}_{2}\right), 33.9\left(\mathrm{CH}_{2}\right), 55.5(\mathrm{CH}), 56.2\left(\mathrm{CH}_{3}\right), 69.0\left(\mathrm{CH}_{2}\right), 85.5$ $(\mathrm{CH}), 91.6(\mathrm{C}), 98.8(\mathrm{C}), 128.0(2 \times \mathrm{CH}), 128.1(2 \times \mathrm{CH}), 130.2(\mathrm{CH})$, $130.4(\mathrm{CH}), 131.41(\mathrm{C}), 131.45(\mathrm{C}), 135.6(2 \times \mathrm{CH}), 135.7(2 \times \mathrm{CH})$, $209.3 \mathrm{ppm}(\mathrm{C})$; IR $\left(\mathrm{CHCl}_{3}, 0.2 \mathrm{~mm}\right): \tilde{n^{-}}=3356,2935,1762 \mathrm{~cm}^{-1}$; MS $\left(\mathrm{ESI}^{+}\right.$ ): $m / z(\%): 477$ (100) $\left[M^{+}+\mathrm{Na}\right]$; HRMS (ESI $\left.{ }^{+}\right): m / z$ calcd for $\mathrm{C}_{26} \mathrm{H}_{34} \mathrm{NaO}_{5} \mathrm{Si}$ : $477.2073\left[M^{+}+\mathrm{Na}\right]$; found: 477.2076; elemental analysis calcd (\%) for $\mathrm{C}_{26} \mathrm{H}_{34} \mathrm{O}_{5} \mathrm{Si}$ (454.63): $\mathrm{C}$ 68.69, $\mathrm{H}$ 7.54; found: $\mathrm{C}$ 68.64, $\mathrm{H} \quad 7.76$. 
4,8-Anhydro-9-O-(3,5-dinitrobenzoyl)-1,6,7-trideoxy-5-O-methyl-d-arabino-nono-2,3-diulose (43): Prepared from $37(65.5 \mathrm{mg}, 0.17 \mathrm{mmol})$ following the general procedure (Method B) using $\mathrm{NaIO}_{4} \quad(111.1 \mathrm{mg}$, $0.52 \mathrm{mmol})$ and $\mathrm{RuO}_{2} \cdot x \mathrm{H}_{2} \mathrm{O}(0.5 \mathrm{mg})$. The reaction mixture was stirred at room temperature in the dark for $30 \mathrm{~min}$. The residue was purified by rapid silica gel column chromatography (EtOAc) to give $43(28.1 \mathrm{mg}$, $0.06 \mathrm{mmol}, 71 \%)$ as a yellow oil. [a $]_{\mathrm{D}}=+47 \mathrm{~cm}^{3} \mathrm{~g}^{-1} \mathrm{dm}^{-1}(c=0.59 \mathrm{in}$ $\left.\mathrm{CHCl}_{3}\right) ;{ }^{1} \mathrm{H} \mathrm{NMR}\left(500 \mathrm{MHz}, \mathrm{CDCl}_{3}\right): \mathrm{d}=1.52(\mathrm{~m}, 1 \mathrm{H}), 1.79-1.88(\mathrm{~m}, 2$ H), $1.94(\mathrm{~m}, 1 \mathrm{H}), 2.28(\mathrm{~s}, 3 \mathrm{H}), 3.28(\mathrm{~s}, 3 \mathrm{H}), 3.88(\mathrm{ddd}, J=7.6,6.4$, $3.6 \mathrm{~Hz}, 1 \mathrm{H}), 4.41(\mathrm{dd}, J=11.7,3.3 \mathrm{~Hz}, 1 \mathrm{H}), 4.48(\mathrm{dd}, J=11.7,7.5 \mathrm{~Hz}$, $1 \mathrm{H}), 4.61(\mathrm{~m}, 1 \mathrm{H}), 5.38(\mathrm{~d}, J=6.4 \mathrm{~Hz}, 1 \mathrm{H}), 9.22 \mathrm{ppm}(\mathrm{m}, 3 \mathrm{H}) ;{ }^{13} \mathrm{C} \mathrm{N} \mathrm{M} \mathrm{R}$ $\left(125.7 \mathrm{MHz}, \mathrm{CDCl}_{3}\right): \mathrm{d}=22.2\left(\mathrm{CH}_{2}\right), 23.1\left(\mathrm{CH}_{2}\right), 23.5\left(\mathrm{CH}_{3}\right), 56.9\left(\mathrm{CH}_{3}\right)$ $67.9\left(\mathrm{CH}_{2}\right), 69.4(\mathrm{CH}), 75.0(\mathrm{CH}), 75.8(\mathrm{CH}), 122.4(\mathrm{CH}), 129.6(2 \times \mathrm{CH})$, 133.8 (C), 148.7 (2× C), 162.5 (C), 197.6 (C), 199.2 ppm (C); IR $\left(\mathrm{CHCl}_{3}\right.$ $0.2 \mathrm{~mm}): \tilde{n}=2945,1732 \mathrm{~cm}^{-1}$; MS (EI): $\mathrm{m} / \mathrm{z}(\%): 410(3)\left[M^{+}\right], 378(4)$ 340 (14), 339 (85); HRMS (EI): $m / z$ calcd for $\mathrm{C}_{17} \mathrm{H}_{18} \mathrm{~N}_{2} \mathrm{O}_{10}: 410.0961\left[M^{+}\right.$ ]; found: 410.0957; elemental analysis calcd (\%) for $\mathrm{C}_{17} \mathrm{H}_{18} \mathrm{~N}_{2} \mathrm{O}_{10}$ (410.33): C 49.76, H 4.42, N 6.83; found: C 49.68, H 4.37, N 6.61.

(5R,6Z)-7-Hydroxy-5-methoxy-2,8-dioxo-6-nonenyl 3,5-dinitrobenzoate (53): A deoxygenated solution of diketone 43 (47.6 $\mathrm{mg}, 0.12 \mathrm{mmol}$ ) in purified $\mathrm{CDCl}_{3}(0.3 \mathrm{~mL})$ was placed in a resonance tube and irradiated with a daylight lamp at $308 \mathrm{C}$ for $6 \mathrm{~h}$. The reaction was monitored by ${ }^{1} \mathrm{H}$ NMR spectroscopy and complete transformation into the photoenol intermediate was observed. The solution was concentrated at low temperature under reduced pressure to give 53, which was not stable enough to with- stand chromatographic purification (silica gel or alumina). Notwithstand- ing, the crude residue (yellowish oil) was pure enough to allow the com- plete analytical and spectroscopic characterization. $[\mathrm{a}]_{\mathrm{D}}=+8 \mathrm{~cm}^{3} \mathrm{~g}^{-1} \mathrm{dm}^{-1}(\mathrm{c}=$ 1.39, $\left.\mathrm{CDCl}_{3}\right) ;{ }^{1} \mathrm{H}$ NMR $\left(400 \mathrm{MHz}, \mathrm{CDCl}_{3}\right): \mathrm{d}=1.94(\mathrm{~m}, 1 \mathrm{H}), 2.03(\mathrm{~m}, 1$ $\mathrm{H}), 2.39(\mathrm{~s}, 3 \mathrm{H}), 2.61(\mathrm{~m}, 2 \mathrm{H}), 3.32(\mathrm{~s}, 3 \mathrm{H}), 4.30$

(ddd, $J=8.2,7.7,5.6 \mathrm{~Hz}, 1 \mathrm{H}), 5.06(\mathrm{~s}, 2 \mathrm{H}), 5.49$ (dd, $J=8.2,1.3 \mathrm{~Hz}, 1 \mathrm{H}$ ), $6.65(\mathrm{~d}, J=1.3 \mathrm{~Hz}, 1 \mathrm{H}), 9.20$ (d, $J=2.1 \mathrm{~Hz}, 2 \mathrm{H}), 9.25 \mathrm{ppm}(\mathrm{dd}, J=2.1$, 2.1, $1 \mathrm{H}) ;{ }^{13} \mathrm{C} \mathrm{NMR}\left(100.6 \mathrm{MHz}, \mathrm{CDCl}_{3}\right): \mathrm{d}=23.1\left(\mathrm{CH}_{3}\right), 28.3\left(\mathrm{CH}_{2}\right), 34.7$ $\left(\mathrm{CH}_{2}\right), 57.0\left(\mathrm{CH}_{3}\right), 69.3\left(\mathrm{CH}_{2}\right), 74.9(\mathrm{CH}), 115.1(\mathrm{CH}), 122.7(\mathrm{CH}), 129.7$ $(2 \times \mathrm{CH}), 133.1$ (C), $148.8(2 \times \mathrm{C}), 148.9$ (C), 161.9 (C), 194.7 (C), $201.3 \mathrm{ppm}^{-}$
$1664 \mathrm{~cm}^{-1}$ (6110 $\left.\mathrm{mol}^{-1} \mathrm{~m}^{3} \mathrm{~cm}^{-1}\right)$; MS (EI): $m / z(\%): 410(<1)\left[M^{+}\right], 378(2), 367(1)$, 335(17); HRMS (EI): $m / z$ : calcd for $\mathrm{C}_{17} \mathrm{H}_{18} \mathrm{~N}_{2} \mathrm{O}_{10}$ : 410.0961 [ $\left.M^{+}\right]$; found: 410.0967; elemental analysis calcd (\%) for $\mathrm{C}_{17} \mathrm{H}_{18} \mathrm{~N}_{2} \mathrm{O}_{10}$ (410.33): $\mathrm{C} 49.76, \mathrm{H}$ 4.42, N 6.83; found: C 49.91, H 4.45, N 6.45 .

4,8-Anhydro-9-O-(tert-butyldiphenylsilyl)-1-deoxy-5,6,7-tri-O-methyl-dglycero-d-talo-nono-2,3-diulosa (58): Prepared from $54 \quad(50 \mathrm{mg}$, $0.103 \mathrm{mmol}$ ) following the general procedure (Method B) using $\mathrm{NaIO}_{4}$ (88 $\mathrm{mg}, 0.412 \mathrm{mmol})$ and $\mathrm{RuO}_{2} \times \mathrm{H}_{2} \mathrm{O}(0.7 \mathrm{mg})$. The reaction mixture was stirred at room temperature in the dark for $1 \mathrm{~h}$. The residue was purified by rapid silica gel column chromatography (hexanes/EtOAc 1:1) to give 58 (28 $\mathrm{mg}, 0.055 \mathrm{mmol}, 53 \%)$ as a yellow oil: $[\mathrm{a}]_{\mathrm{D}}=+16 \mathrm{~cm}^{3} \mathrm{~g}^{-1} \mathrm{dm}^{-1}(c=1.50 \mathrm{in}$ $\left.\mathrm{CHCl}_{3}\right) ;{ }^{1} \mathrm{H} \mathrm{N} \mathrm{MR}\left(400 \mathrm{MHz}, \mathrm{C}_{6} \mathrm{D}_{6}\right): \mathrm{d}=1.20(\mathrm{~s}, 9 \mathrm{H}), 1.86(\mathrm{~s}, 3 \mathrm{H}), 3.18(\mathrm{~s}$, $3 \mathrm{H}$ ), 3.19 (s, $3 \mathrm{H}$ ), 3.33 (s, $3 \mathrm{H}$ ), 3.43 (dd, $J=8.2,3.2 \mathrm{~Hz}, 1 \mathrm{H}$ ), 3.81 (dd, $J=$ 8.0, $8.0 \mathrm{~Hz}, 1 \mathrm{H}$ ), 4.00 (dd, $J=3.4,3.4 \mathrm{~Hz}, 1 \mathrm{H}), 4.02-4.06(\mathrm{~m}, 3 \mathrm{H}), 5.28(\mathrm{~d}$, $J=3.4 \mathrm{~Hz}, 1 \mathrm{H}), 7.21-7.89 \mathrm{ppm}(\mathrm{m}, 10 \mathrm{H}) ;{ }^{13} \mathrm{C} \mathrm{NMR}\left(100.6 \mathrm{MHz}, \mathrm{C}_{6} \mathrm{D}_{6}\right)$ : $\mathrm{d}=19.6(\mathrm{C}), 24.0\left(\mathrm{CH}_{3}\right), 27.1\left(3 \times \mathrm{CH}_{3}\right), 57.5\left(\mathrm{CH}_{3}\right), 57.7\left(\mathrm{CH}_{3}\right), 59.8$ $\left(\mathrm{CH}_{3}\right), 63.8\left(\mathrm{CH}_{2}\right), 74.5(\mathrm{CH}), 75.8(\mathrm{CH}), 76.3(\mathrm{CH}), 78.2(\mathrm{CH}), 81.2(\mathrm{CH})$, 128.1-132.6 (10 × CH), 133.9 (C), 134.2 (C), 198.2 (C), 198.9 ppm (C); IR (film): $\tilde{\mathrm{n}^{2}}=2933,1718 \mathrm{~cm}^{-1}$; MS $\left(\mathrm{ESI}^{+}\right): \mathrm{m} / \mathrm{z} \quad$ (\%): $569 \quad$ (100) $\left[\mathrm{M}^{+}+\mathrm{MeOH}+\mathrm{Na}\right] ;$ HRMS $\left(\mathrm{ESI}^{+}\right): \mathrm{m} / \mathrm{z}$ : calcd for

$\mathrm{C}_{2} \mathrm{H}{ }_{42} \mathrm{NaO}$ Sii: $569.2547\left[\mathrm{M}^{+}+\mathrm{MeOH}+\mathrm{Na}\right]$; found: 569.2554 ; elemental analysis calcd (\%) for $\mathrm{C}_{28} \mathrm{H}_{38} \mathrm{O}_{7} \mathrm{Si}$ (514.68): $\mathrm{C}$ 65.34, $\mathrm{H}$ 7.44; found: $\mathrm{C}$ 65.26, H 7.49.

Photocyclization of 58: A deoxygenated solution of diketone 58 (40 mg, $0.078 \mathrm{mmol})$ in dry $\mathrm{C}_{6} \mathrm{D}_{6}(1 \mathrm{~mL})$, was placed in a resonance tube and irradiated with a daylight lamp at $30 \mathrm{8C}$. The reaction was monitored by ${ }^{1} \mathrm{H}$ NMR spectroscopy, complete transformation of the starting material was accomplished after $18 \mathrm{~h}$. The solution was then heated at $50 \mathrm{8C}$ in the dark for $2 \mathrm{~h}$, and concentrated under reduced pressure. The crude residue in $\mathrm{CH}_{2} \mathrm{Cl}_{2}(0.7 \mathrm{~mL})$ was acetylated at $08 \mathrm{C}$ with $\mathrm{Ac}_{2} \mathrm{O}(30 \mathrm{~mL}, 0.778 \mathrm{mmol})$ and 4-dimethylaminopyridine (DMAP; $28 \mathrm{mg}, 0.233 \mathrm{mmol}$ ) for $2 \mathrm{~h}$. The mixture was concentrated under reduced pressure and the residue puri- fied by silica gel column chromatography (hexanes/Et0Ac 7:3) to give $(2 S, 3 \mathrm{a} R, 4 R, 5 R, 6 S, 6 \mathrm{a} S)-6 \mathrm{a}-[($ tert-butyldiphenylsilyl)oxy]methyl-4,5,6-trimethoxy-2-methyl-3-oxohexahydro- $2 H$-cyclopenta[b]furan-2-yl acetate (62) (13 $\mathrm{mg}, 0.023 \mathrm{mmol}, 30 \%$ ) as a colorless oil. The sensitive photoenol intermediate was detected by NMR spectroscopy but in this case could not be obtained in pure state. Only clearly distinguished signals from the re- action mixture are reported: ${ }^{1} \mathrm{H} N \mathrm{MR}\left(400 \mathrm{MHz}, \mathrm{C}_{6} \mathrm{D}_{6}\right): \mathrm{d}=1.26(\mathrm{~s}, 9 \mathrm{H}), 1.77(\mathrm{~s}$, $3 \mathrm{H}), 2.90$ (s, 3H), 3.10 (s, 3H), $3.6(\mathrm{~s}, 3 \mathrm{H}), 4.71$ (d, $J=18.3 \mathrm{~Hz}$, $1 \mathrm{H}$ ), 4.85 (d, $J=18.5 \mathrm{~Hz}, 1 \mathrm{H}$ ), 5.33 (br d, $J=9.3 \mathrm{~Hz}, 1 \mathrm{H}$ ), $6.73 \mathrm{ppm}(\mathrm{brs}$, $1 \mathrm{H}, \mathrm{OH})$.

Compound 62: $[\mathrm{a}]_{\mathrm{D}}=-4 \mathrm{~cm}^{3} \mathrm{~g}^{-1} \mathrm{dm}^{-1}\left(\mathrm{c}=1.00 \mathrm{in} \mathrm{CHCl}_{3}\right) ;{ }^{1} \mathrm{H} \mathrm{NMR}$ $\left(500 \mathrm{MHz}, \mathrm{CDCl}_{3}\right): \mathrm{d}=1.07(\mathrm{~s}, 9 \mathrm{H}), 1.52(\mathrm{~s}, 3 \mathrm{H}), 1.85(\mathrm{~s}, 3 \mathrm{H}), 3.41(\mathrm{~d}$, $J=1.9 \mathrm{~Hz}, 1 \mathrm{H}$ ), 3.431 (dd, $J=7.9,4.7 \mathrm{~Hz}, 1 \mathrm{H}), 3.434(\mathrm{~s}, 3 \mathrm{H}), 3.46$ (s, $3 \mathrm{H}), 3.52(\mathrm{~s}, 3 \mathrm{H}), 3.72(\mathrm{~d}, J=10.4 \mathrm{~Hz}, 1 \mathrm{H}), 3.79(\mathrm{dd}, J=4.7,2.1 \mathrm{~Hz}, 1 \mathrm{H})$, $3.87(\mathrm{~d}, J=10.1 \mathrm{~Hz}, 1 \mathrm{H}), 4.19(\mathrm{~d}, J=8.2 \mathrm{~Hz}, 1 \mathrm{H}), 7.35-7.70 \mathrm{ppm}(\mathrm{m}$, $10 \mathrm{H}) ;{ }^{13} \mathrm{CNMR}\left(125.7 \mathrm{MHz}, \mathrm{CDCl}_{3}\right): \mathrm{d}=19.3$ (C), $20.4\left(\mathrm{CH}_{3}\right), 22.4$ $\left(\mathrm{CH}_{3}\right), 26.8\left(3 \times \mathrm{CH}_{3}\right), 51.8(\mathrm{CH}), 57.5\left(\mathrm{CH}_{3}\right), 58.0\left(\mathrm{CH}_{3}\right), 60.1\left(\mathrm{CH}_{3}\right), 64.8$ $\left(\mathrm{CH}_{2}\right), 79.5(\mathrm{CH}), 82.4(\mathrm{CH}), 85.6(\mathrm{CH}), 88.3(\mathrm{C}), 101.1(\mathrm{C}), 127.6-135.7$ $(10 \times \mathrm{CH}), 132.9(\mathrm{C}), 133.0(\mathrm{C}), 169.7(\mathrm{C}), 207.7 \mathrm{ppm}(\mathrm{C})$; IR (film): $\tilde{\mathrm{n}}=$ 2921, 1763, 1743 $\mathrm{cm}^{-1}$; MS (ESI $\left.{ }^{+}\right): m / z(\%): 579$ (100) $\left[M^{+}+\mathrm{Na}\right]$; HRMS $\left(\mathrm{ESI}^{+}\right.$): $\mathrm{m} / \mathrm{z}$ calcd for $\mathrm{C}_{30} \mathrm{H}_{40} \mathrm{NaO}_{8} \mathrm{Si} ; 579.2390\left[M^{+}+\mathrm{Na}\right]$; found: 579.2396 ; elemental analysis calcd (\%) for $\mathrm{C}_{30} \mathrm{H}_{40} \mathrm{O}_{8} \mathrm{Si}$ (558.72): $\mathrm{C}$ 64.72, H 7.24; found: C $66.70, \mathrm{H} 7.05$.

4,8-Anhydro-1-deoxy-5,6,7,9-tetra-O-methyl-d-glycero-1-gluco-nono-2,3diulose (59): Prepared from 55 (150 $\mathrm{mg}, 0.581 \mathrm{mmol}$ ) following the general procedure (Method A) using dimethyl sulfide ( $85 \mathrm{~mL}, 1.162 \mathrm{mmol}$ ). The mixture was stirred at room temperature in the dark for $3 \mathrm{~h}$ and concentrated under reduced pressure. The residue was purified by rapid silica gel column chromatography (hexanes/EtOAc 1:1) to give 59 as a yellow oil ( $98 \mathrm{mg}, 0.338 \mathrm{mmol}, 58 \%$ ), which was unstable on silica gel. However, a small analytical sample could be obtained by rapid silica gel column chromatography (hexanes/EtOAc 7:3). [a] $]_{\mathrm{D}}=-30 \mathrm{~cm}^{3} \mathrm{~g}^{-1} \mathrm{dm}^{-1}$ $\left(c=2.14\right.$ in $\left.\mathrm{CHCl}_{3}\right) ;{ }^{1} \mathrm{H} \mathrm{NMR}\left(400 \mathrm{MHz} \mathrm{CDCl}_{3}\right): \mathrm{d}=2.35(\mathrm{~s}, 3 \mathrm{H}), 3.32(\mathrm{~s}$ $3 \mathrm{H}), 3.36(\mathrm{~s}, 3 \mathrm{H}), 3.39-3.56(\mathrm{~m}, 3 \mathrm{H}), 3.44(\mathrm{~s}, 3 \mathrm{H}), 3.51(\mathrm{~s}, 3 \mathrm{H}), 3.83(\mathrm{dd}$, $J=4.1,1.9 \mathrm{~Hz}, 1 \mathrm{H}), 4.01(\mathrm{dd}, J=2.2,1.9 \mathrm{~Hz}, 1 \mathrm{H}), 4.19$ (dd, $J=4.7$, $4.4 \mathrm{~Hz}, 1 \mathrm{H}), \quad 5.07 \mathrm{ppm} \quad(\mathrm{d}, J=2.2 \mathrm{~Hz}, 1 \mathrm{H}) ;{ }^{13} \mathrm{C} \mathrm{NMR}(100.6 \mathrm{MHz}$, $\left.\mathrm{CDCl}_{3}\right): \mathrm{d}=25.6\left(\mathrm{CH}_{3}\right), 58.3\left(\mathrm{CH}_{3} 58.4(\mathrm{CH}), 59.8(\mathrm{CH}), 60.0(\mathrm{CH}), 3\right.$ $73.0\left(\mathrm{CH}_{2}\right), 80.4(\mathrm{CH}), 84.7(\mathrm{CH}), 85.2(\mathrm{CH}), 85.9(\mathrm{CH}), 87.6(\mathrm{CH}), 196.9$ (C), $199.7 \mathrm{ppm}$ (C); IR (film): $\tilde{n}=2931,1728,1714 \mathrm{~cm}^{-1}$; MS (EI): $\mathrm{m} / \mathrm{z}$ (\%): 291 (34) $\left[M^{+}+\mathrm{H}\right]$; HRMS (EI): $m / z$ calcd for $\mathrm{C}_{13} \mathrm{H}_{23} \mathrm{O}_{7}: 291.1444$ $\left[M^{+}+\mathrm{H}\right]$; found: 291.1414; elemental analysis calcd (\%) for $\mathrm{C}_{13} \mathrm{H}_{22} \mathrm{O}_{7}$ (290.31):C 53.78, H 7.64; found: C 53.67,H 7.62.

Photolysis of 59: A deoxygenated solution of diketone $59(25 \mathrm{mg}$, $0.086 \mathrm{mmol})$ in dry $\mathrm{C}_{6} \mathrm{D}_{6}(0.6 \mathrm{~mL})$, was placed in a resonance tube and irradiated with a daylight lamp at $30 \mathrm{8C}$. The reaction was monitored by ${ }^{1} \mathrm{H}$ NMR spectroscopy, complete consumption of starting material was observed after $2 \mathrm{~h}$, leading to exclusive formation of intermediate photoenol. The solution could be concentrated at low temperature under reduced pressure but the photoenol was not stable enough to withstand chromatographic purification (silica gel or alumina). After silica gel column chromatography (hexanes/EtOAc 40:60) only the known 2,3,4,6- tetra- $O$ methyl-d-galactono-1,5-lactone ${ }^{[46]}(16 \mathrm{mg}, 0.068 \mathrm{mmol}, 80 \%)$ could be obtained.

4,8-Anhydro-9-O-(tert-butyldiphenylsilyl)-1-deoxy-5,6,7-tri-O-methyl-dglycero-1-gluco-nono-2,3-diulose (60): Prepared from $56(50 \mathrm{mg}$, $0.104 \mathrm{mmol}$ ) following the general procedure (Method B) using NaIO $(89 \mathrm{mg}, 0.416 \mathrm{mmol})$ and $\mathrm{RuO}_{2} \cdot x \mathrm{H}_{2} \mathrm{O}(0.9 \mathrm{mg})$. The reaction mixture was stirred at room temperature in the dark for $20 \mathrm{~min}$. The residue was purified by rapid silica gel column chromatography (hexanes/EtOAc 4:6) to give 60 (34 mg, $0.066 \mathrm{mmol}, 64 \%$ ) as a yellow oil: [a $]_{\mathrm{D}}=+61 \mathrm{~cm}^{3} \mathrm{~g}^{-1} \mathrm{dm}^{-1}$ $\left(c=0.25\right.$ in $\left.\mathrm{CHCl}_{3}\right) ;{ }^{1} \mathrm{H} \mathrm{NMR}\left(500 \mathrm{MHz} \mathrm{CDCl}_{3}\right): \mathrm{d}=1.07(\mathrm{~s}, 9 \mathrm{H}), 2.28$ (s, $3 \mathrm{H}$ ), 3.37 (s, $3 \mathrm{H}$ ), 3.41 (dd, $J=8.5,2.6 \mathrm{~Hz}, 1 \mathrm{H}), 3.50$ (s,

$3 \mathrm{H}), 3.53(\mathrm{~s}, 3 \mathrm{H}), 3.79(\mathrm{~d}, J=2.4 \mathrm{~Hz}, 1 \mathrm{H}), 3.81(\mathrm{~d}, J=1.1 \mathrm{~Hz}, 1 \mathrm{H}), 3.91$ (dd, $J=2.6,2.4 \mathrm{~Hz}, 1 \mathrm{H}$ ), 3.94 (dd, $J=8.5,6.9 \mathrm{~Hz}, 1 \mathrm{H}$ ), $4.44(\mathrm{~m}, 1 \mathrm{H}), 5.38$ (d, $J=6.9 \mathrm{~Hz}, 1 \mathrm{H}), 7.35-7.77 \mathrm{ppm}(\mathrm{m}, 10 \mathrm{H}) ;{ }^{13} \mathrm{C} \mathrm{N} \mathrm{MR}(100.6 \mathrm{MHz}$, $\left.\mathrm{CDCl}_{3}\right): \mathrm{d}=19.2\left(\mathrm{CH}_{3}\right), 23.5(\mathrm{C}), 26.9\left(3 \times \mathrm{CH}_{3}\right), 57.8\left(\mathrm{CH}_{3}\right), 59.5\left(\mathrm{CH}_{3}\right)$, $60.7\left(\mathrm{CH}_{3}\right), 61.2\left(\mathrm{CH}_{2}\right), 71.9(\mathrm{CH}), 74.6(\mathrm{CH}), 75.4(\mathrm{CH}), 78.4(\mathrm{CH}), 81.2$ 
(CH), 127.7-135.6 (10× CH), $133.3(\mathrm{C}), 133.4(\mathrm{C}), 197.0(\mathrm{C}), 200.1 \mathrm{ppm}$ (C); IR (film): $\tilde{n}=2936,1716 \mathrm{~cm}^{-1}$; MS (ESI $\left.{ }^{+}\right): m / z(\%): 569(100)\left[M^{+}\right.$ $+\mathrm{MeOH}+\mathrm{Na}$ ]; HRMS (ESI ${ }^{+}$): $m / z$ calcd for $\mathrm{C}_{29} \mathrm{H}_{42} \mathrm{NaO}_{8} \mathrm{Si}: 569.2547$ $\left[\mathrm{M}^{+}+\mathrm{MeOH}+\mathrm{Na}\right]$; found: 569.2556 ; elemental analysis calcd (\%) for $\mathrm{C}_{28} \mathrm{H}_{38} \mathrm{O}_{7} \mathrm{Si}$ (514.68): C 65.34, H 7.44; found: C $65.49, \mathrm{H} 7.36$.

Photocyclization of 60: A deoxygenated solution of diketone 60 (28 mg, $0.054 \mathrm{mmol})$ in dry $\mathrm{C}_{6} \mathrm{D}_{6}(0.7 \mathrm{~mL})$, was placed in a resonance tube and irradiated with a daylight lamp at $308 \mathrm{C}$. The reaction was monitored by ${ }^{1} \mathrm{H}$ N M R spectroscopy; complete transformation into the enol intermedi- ate was observed after $2.5 \mathrm{~h}$. The solution was heated at $60 \mathrm{8C}$ in the dark for 1.5 $\mathrm{h}$ and concentrated under reduced pressure. The residue was dis- solved in $\mathrm{CH}_{2} \mathrm{Cl}_{2}(0.6 \mathrm{~mL})$, cooled at $08 \mathrm{C}$, and acetylated with $\mathrm{Ac}_{2} \mathrm{O} \quad(20 \mathrm{~mL}, 0.163$ $\mathrm{mmol}$ ) and D M A P (20 mg, $0.163 \mathrm{mmol})$. The solution was stirred at room temperature for $2 \mathrm{~h}$, concentrated under reduced pres- sure, and the residue purified by silica gel column chromatography (hex- anes/Et0Ac 7:3) to give $(2 S, 3 a R, 4 S, 5 R, 6 R, 6 \mathrm{a} S)-6 \mathrm{a}-[($ tert-butyldiphenylsi- lyl)oxy]methyl-4,5,6trimethoxy-2-methyl-3-oxohexahydro- $2 H$-cyclopen- ta[b] furan-2-yl acetate (64) and $(2 R, 3 \mathrm{a} S, 4 S, 5 R, 6 R, 6 \mathrm{a} R)$-6a-[(tert-butyldi- phenylsilyl)oxy]methyl4,5,6-trimethoxy-2-methyl-3-oxohexahydro- $2 H$-cy- clopenta[ $b]$ furan-2-yl acetate (63) (21 mg, $0.038 \mathrm{mmol}$, d.r. 2:1,69\%). The sensitive photoenol intermediate was detected by N M R spectroscopy but in this case could not be obtained in pure state. Only clearly distin- guished signals from the reaction mixture are reported. ${ }^{1} \mathrm{H} \mathrm{N} \mathrm{MR}\left(400 \mathrm{M} \mathrm{Hz}, \mathrm{C}_{6} \mathrm{D}_{6}\right): \mathrm{d}=1.25(\mathrm{~s}, 9$ H), $1.85(\mathrm{~s}, 3 \mathrm{H}), 2.89(\mathrm{~s}, 3 \mathrm{H}), 3.13(\mathrm{~s}, 3 \mathrm{H}), 3.15(\mathrm{~s}, 3 \mathrm{H}), 4.68(\mathrm{~d}, J=18.3 \mathrm{~Hz}$, $1 \mathrm{H}), 4.74(\mathrm{~d}, J=18.6 \mathrm{~Hz}, 1 \mathrm{H}), 5.54(\mathrm{dd}, J=9.0,1.1 \mathrm{~Hz}, 1 \mathrm{H}), 6.84 \mathrm{ppm}(\mathrm{d}$, $J=1.3 \mathrm{~Hz}, \mathrm{OH}) ;{ }^{13} \mathrm{C} \mathrm{NMR}\left(100.6 \mathrm{MHz}, \mathrm{C}_{6} \mathrm{D}_{6}\right): \mathrm{d}=19.6(\mathrm{C}), 22.8\left(\mathrm{CH}_{3}\right)$, $27.0\left(3 \times \mathrm{CH}_{3}\right), 57.0\left(\mathrm{CH}_{3}\right), 58.2\left(\mathrm{CH}_{3}\right), 59.9\left(\mathrm{CH}_{3}\right), 69.5\left(\mathrm{CH}_{2}\right), 111.7$ (CH), 149.9 (C), $194.6(\mathrm{C}), 206.4 \mathrm{ppm}$ (C).

Compound 63: colorless oil. [a $]_{\mathrm{D}}=+14 \mathrm{~cm}^{3} \mathrm{~g}^{-1} \mathrm{dm}^{-1}\left(c=0.28\right.$ in $\left.\mathrm{CHCl}_{3}\right)$; ${ }^{1} \mathrm{H} \mathrm{NMR}(400 \mathrm{MHz}, \mathrm{CDCl}): \mathrm{d}=1.08(\mathrm{~s}, 9 \mathrm{H}), 1.54(\mathrm{~s}, 3 \mathrm{H}), 1.87(\mathrm{~s}, 3 \mathrm{H})$, $3.27(\mathrm{~d}, J=2.9 \mathrm{~Hz}, 1 \mathrm{H}), 3.36(\mathrm{~s}, 3 \mathrm{H}), 3.45(\mathrm{~s}, 3 \mathrm{H}), 3.48(\mathrm{~s}, 3 \mathrm{H}), 3.70$ (d, $J=10.3 \mathrm{~Hz}, 1 \mathrm{H}$ ), 3.77 (dd, $J=4.2,4.2 \mathrm{~Hz}, 1 \mathrm{H}$ ), 3.81 (dd, $J=4.8,2.9 \mathrm{~Hz}$, $1 \mathrm{H}), 3.88(\mathrm{~d}, J=10.1 \mathrm{~Hz}, 1 \mathrm{H}), 4.06(\mathrm{~d}, J=4.0 \mathrm{~Hz}, 1 \mathrm{H}), 7.36-7.72 \mathrm{ppm}$ $(\mathrm{m}, 10 \mathrm{H}) ;{ }^{13} \mathrm{C} \mathrm{NMR}(100.6 \mathrm{MHz}, \mathrm{CDCl}) \mathrm{d}=19.3(\mathrm{C}), 20.7(\mathrm{CH}), 22.7$

$\left(\mathrm{CH}_{3}\right), 26.8\left(3 \times \mathrm{CH}_{3}\right), 53.3(\mathrm{CH}), 57.7\left(\mathrm{CH}_{3}\right), 57.8\left(\mathrm{CH}_{3}\right), 59.9\left(\mathrm{CH}_{3}\right), 65.4$ $\left(\mathrm{CH}_{2}\right), 81.2(\mathrm{CH}), 83.3(\mathrm{CH}), 83.9(\mathrm{CH}), 89.2(\mathrm{C}), 102.0(\mathrm{C}), 127.7-135.7$ $(10 \times \mathrm{CH}), 132.97(\mathrm{C}), 133.00(\mathrm{C}), 169.8(\mathrm{C}), 207.4 \mathrm{ppm}(\mathrm{C})$; IR (film): $\tilde{n}=2930,1766,1745 \mathrm{~cm}^{-1}$; MS (ESI $\left.{ }^{+}\right): m / z(\%): 579(100)\left[M^{+}+\mathrm{Na}\right]$; HRMS $\left(\mathrm{ESI}^{+}\right.$): $m / z$ calcd for $\mathrm{C}_{30} \mathrm{~N}_{48} \mathrm{O} \mathrm{Si}{ }_{8} 579.2390\left[M^{+}+\mathrm{Na}\right]$; found: 579.2393; elemental analysis calcd (\%) for $\mathrm{C}_{30} \mathrm{H}_{40} \mathrm{O}_{8} \mathrm{Si}(556.72): \mathrm{C}$ 64.72, $\mathrm{H}$ 7.24; found: C $64.75, \mathrm{H} 7.16$.

Compound 64: colorless oil; [a] $\overline{\mathrm{D}}+29 \mathrm{~cm}^{3} \mathrm{~g}^{-1} \mathrm{dm}^{-1}(c=0.38$ in CHCl $)$; $_{3}$ ${ }^{1} \mathrm{H} \mathrm{NMR}(500 \mathrm{MHz}, \mathrm{CDCl}) \mathrm{d}=1.06(\mathrm{~s}, 9 \mathrm{H}), 1.59(\mathrm{~s}, 3 \mathrm{H}), 1.63(\mathrm{~s}, 3 \mathrm{H})$, $3.14(\mathrm{~d}, J=9.5 \mathrm{~Hz}, 1 \mathrm{H}), 3.47(\mathrm{~s}, 3 \mathrm{H}), 3.50(\mathrm{~s}, 3 \mathrm{H}), 3.58(\mathrm{~s}, 3 \mathrm{H}), 3.71(\mathrm{dd}$, $J=8.2,3.5 \mathrm{~Hz}, 1 \mathrm{H}), 3.92(\mathrm{~d}, J=11.0 \mathrm{~Hz}, 1 \mathrm{H}), 3.94(\mathrm{~d}, J=3.5 \mathrm{~Hz}, 1 \mathrm{H})$, 4.00 (d, $J=11.4 \mathrm{~Hz}, 1 \mathrm{H}), 4.07$ (dd, $J=9.5,8.2 \mathrm{~Hz}, 1 \mathrm{H}), 7.35-7.72 \mathrm{ppm}$ $(\mathrm{m}, 10 \mathrm{H}) ;{ }^{13} \mathrm{C} \mathrm{NMR}\left(125.7 \mathrm{MHz}, \mathrm{CDCl}_{3}\right): \mathrm{d}=19.4(\mathrm{C}), 20.8\left(\mathrm{CH}_{3}\right), 22.0$ $\left(\mathrm{CH}_{3}\right), 26.9\left(3 \times \mathrm{CH}_{3}\right), 49.6(\mathrm{CH}), 58.9\left(\mathrm{CH}_{3}\right), 59.2\left(\mathrm{CH}_{3}\right), 60.9\left(\mathrm{CH}_{3}\right), 64.9$ $\left(\mathrm{CH}_{2}\right), 82.0(\mathrm{CH}), 83.8(\mathrm{CH}), 85.7(\mathrm{CH}), 92.2(\mathrm{C}), 101.9(\mathrm{C}), 127.6-137.8$ $(10 \times \mathrm{CH}), 129.59(\mathrm{C}), 129.60(\mathrm{C}), 169.5(\mathrm{C}), 205.5 \mathrm{ppm}(\mathrm{C})$; IR (film): $\tilde{n}=2923,1766,1747 \mathrm{~cm}^{-1}$; MS $\left(\mathrm{ESI}^{+}\right): \mathrm{m} / \mathrm{z}(\%): 579(100)\left[\mathrm{M}^{+}+\mathrm{Na}\right]$; HRMS (EI): $m / z$ calcd for $\mathrm{C}_{30} \mathrm{H}_{40} \mathrm{NaO}_{8} \mathrm{Si}: 579.2490\left[M^{+}+\mathrm{Na}\right]$; found: 579.2401; elemental analysis calcd (\%) for $\mathrm{C}_{30} \mathrm{H}_{40} \mathrm{O}_{8} \mathrm{Si}$ (556.72): C 64.72, H 7.24; found: C 64.35, H 7.11.

9-O-Acetyl-4,8-anhydro-1-deoxy-5,6,7-tri-O-methyl-d-glycero-1-gluconono-2,3-diulose (61): Prepared from 57 (70 $\mathrm{mg}, 0.244 \mathrm{mmol})$ following the general procedure (Method $\mathrm{B}$ ) using $\mathrm{NaIO}_{4}(156 \mathrm{mg}, 0.732 \mathrm{mmol})$ and $\mathrm{RuO}_{2} \cdot x \mathrm{H}_{2} \mathrm{O}(1.2 \mathrm{mg})$. The reaction mixture was stirred at room temperature in the dark for $40 \mathrm{~min}$. The residue was purified by rapid silica gel column chromatography (hexanes/EtOAc $4: 6)$ to give $61(37 \mathrm{mg}$, $0.116 \mathrm{mmol}, 48 \%)$ as a yellow oil. [a] $]_{\mathrm{D}}=+82 \mathrm{~cm}^{3} \mathrm{~g}^{-1} \mathrm{dm}^{-1}(c=0.71$ in $\left.\mathrm{CHCl}_{3}\right) ;{ }^{1} \mathrm{H} \mathrm{NMR}\left(500 \mathrm{MHz}, \mathrm{CDCl}_{3}\right): \mathrm{d}=2.05(\mathrm{~s}, 3 \mathrm{H}), 2.28(\mathrm{~s}, 3 \mathrm{H}), 3.34$ $(\mathrm{s}, 3 \mathrm{H}), 3.47(\mathrm{~m}, 1 \mathrm{H}), 3.48(\mathrm{~s}, 3 \mathrm{H}), 3.49(\mathrm{~s}, 3 \mathrm{H}), 3.72(\mathrm{dd}, J=3.2,3.2 \mathrm{~Hz}, 1$ H), 3.98 (dd, $J=6.9,5.0 \mathrm{~Hz}, 1 \mathrm{H}$ ), 4.15 (dd, $J=12.0,3.5 \mathrm{~Hz}, 1 \mathrm{H}$ ), $4.42-4.51$ $(\mathrm{m}, 2 \mathrm{H}), 5.39 \mathrm{ppm}(\mathrm{d}, J=5.4 \mathrm{~Hz}, 1 \mathrm{H}) ;{ }^{13} \mathrm{C} \mathrm{NMR}\left(100.6 \mathrm{MHz}, \mathrm{C}_{6} \mathrm{D}_{6}\right): \mathrm{d}=$ $20.8\left(\mathrm{CH}_{3}\right), 23.7\left(\mathrm{CH}_{3}\right), 58.4\left(\mathrm{CH}_{3}\right), 59.1\left(\mathrm{CH}_{3}\right), 59.6\left(\mathrm{CH}_{3}\right), 61.7\left(\mathrm{CH}_{2}\right), 71.6$ $(\mathrm{CH}), 73.4(\mathrm{CH}), 75.2(\mathrm{CH}), 77.9(\mathrm{CH}), 78.7(\mathrm{CH}), 170.9(\mathrm{C}), 197.1(\mathrm{C})$, $198.5 \mathrm{ppm}(\mathrm{C}) ; \mathrm{IR}$ (film): $\tilde{\mathrm{n}}=2936,1739 \mathrm{~cm}^{-1}$; $\mathrm{MS}\left(\mathrm{ESI}^{+}\right): \mathrm{m} / \mathrm{z}$
(\%): 373(100) $\left[M^{+}+\mathrm{MeOH}+\mathrm{Na}\right], 341(21)\left[\mathrm{M}^{+}+\mathrm{Na}\right] ; \mathrm{HRMS}\left(\mathrm{ESI}^{+}\right)$: $\mathrm{m} / \mathrm{z}$ : calcd for $\mathrm{C}_{15} \mathrm{H}_{26} \mathrm{NaO}_{9}$ : $373.1475\left[M^{+}+\mathrm{MeOH}+\mathrm{Na}\right]$; found: 373.1465 ; elemental analysis calcd (\%) for $\mathrm{C}_{14} \mathrm{H}_{22} \mathrm{O}_{8}$ (318.32): $\mathrm{C}$ 52.82, $\mathrm{H}$ 6.97; found: C 52.95, H 6.89 .

Photocyclization of 61: A deoxygenated solution of diketone 61 (14 mg, $0.044 \mathrm{mmol})$ in dry $\mathrm{C}_{6} \mathrm{D}_{6}(0.5 \mathrm{~mL})$, was placed in a resonance tube and irradiated with a daylight lamp at $308 \mathrm{C}$. The reaction was monitored by ${ }^{1} \mathrm{H}$ N MR spectroscopy; complete transformation into the enol intermedi- ate was observed after $2.5 \mathrm{~h}$. The solution was heated at $708 \mathrm{C}$ in the dark for $3 \mathrm{~h}$ and concentrated under reduced pressure. The residue was dis- solved in $\mathrm{CH}_{2} \mathrm{Cl}_{2}(0.4 \mathrm{~mL})$, cooled at $08 \mathrm{C}$, and acetylated with $\mathrm{Ac}_{2} \mathrm{O} \quad(12 \mathrm{~mL}, 0.132$ $\mathrm{mmol}$ ) and D M AP (16 mg, $0.132 \mathrm{mmol}$ ). The solution was stirred at room temperature for $2 \mathrm{~h}$, concentrated under reduced pres- sure, and the residue purified by silica gel column chromatography (hex- anes/EtOAc 7:3) to give $(2 S, 3 \mathrm{a} R, 4 S, 5 R, 6 R, 6 \mathrm{a} S)$-6a-(acetyloxy)methyl- 4,5,6-trimethoxy-2methyl-3-oxohexahydro- $2 H$-cyclopenta[ $b]$ furan-2-yl acetate (66) and $(2 R, 3 \mathrm{a} S, 4 S, 5 R, 6 R, 6 \mathrm{a} R)$-6a-(acetyloxy)methyl-4,5,6-trime- thoxy-2-methyl3-oxohexahydro- $2 H$-cyclopenta[b]furan-2-yl acetate $(65)(12 \mathrm{mg}, 0.033$ mmol, d.r. $3: 1,76 \%$ from 61 ). The sensitive photoenol in- termediate was detected by N M R spectroscopy but in this case could not be obtained in pure state. 0 nly clearly distinguished signals from the re- action mixture are reported: ${ }^{1} \mathrm{H}$ NMR $\left(400 \mathrm{MHz}, \mathrm{C}_{6} \mathrm{D}_{6}\right): \mathrm{d}=1.79(\mathrm{~s}, 3 \mathrm{H}), 1.87(\mathrm{~s}, 3 \mathrm{H}), 3.13(\mathrm{~s}$, $3 \mathrm{H}), 3.14(\mathrm{~s}, 3 \mathrm{H}), 3.24(\mathrm{~s}, 3 \mathrm{H}), 4.92(\mathrm{~d}, J=17.5 \mathrm{~Hz}, 1 \mathrm{H}), 5.07(\mathrm{~d}, J=17.5$ $\mathrm{Hz}, 1 \mathrm{H}), 5.52(\mathrm{brd}, J=9.3 \mathrm{~Hz}, 1 \mathrm{H}), 6.89 \mathrm{ppm}(\mathrm{br} \mathrm{s}, 1 \mathrm{H}, \mathrm{OH}) ;{ }^{13} \mathrm{C} \mathrm{N} \mathrm{M} \mathrm{R}$ $\left(100.6 \mathrm{MHz}, \mathrm{C}_{6} \mathrm{D}_{6}\right): \mathrm{d}=20.1\left(\mathrm{CH}_{3}\right), 22.7\left(\mathrm{CH}_{3}\right), 57.1\left(\mathrm{CH}_{3}\right), 58.7\left(\mathrm{CH}_{3}\right)$, $60.0\left(\mathrm{CH}_{3}\right), 67.7\left(\mathrm{CH}_{2}\right), 111.6(\mathrm{CH}), 150.0(\mathrm{C}), 169.8(\mathrm{C}), 194.6(\mathrm{C}), 202.7$ $\operatorname{ppm}(\mathrm{C})$.

Compound 65: colorless oil, unstable, and decomposed on standing at room temperature preventing us from obtaining good $1 \mathrm{D}$ and 2D NMR spectra. $[\mathrm{a}]_{\mathrm{D}}=+15 \mathrm{~cm}^{3} \mathrm{~g}^{-1} \mathrm{dm}^{-1}\left(c=0.56\right.$ in $\left.\mathrm{CHCl}_{3}\right) ;{ }^{1} \mathrm{H} \mathrm{NMR}(500 \mathrm{MHz}$, $\left.\mathrm{CDCl}_{3}\right): \mathrm{d}=1.57(\mathrm{~s}, 3 \mathrm{H}), 2.04(\mathrm{~s}, 3 \mathrm{H}), 2.11(\mathrm{~s}, 3 \mathrm{H}), 3.29(\mathrm{~d}, \quad J=2.8 \mathrm{~Hz}$, $1 \mathrm{H}), 3.34(\mathrm{~s}, 3 \mathrm{H}), 3.43(\mathrm{~s}, 3 \mathrm{H}), 3.51(\mathrm{~s}, 3 \mathrm{H}), 3.71(\mathrm{dd}, J=4.1,4.1 \mathrm{~Hz}$, $1 \mathrm{H}), 3.76(\mathrm{~d}, J=4.1 \mathrm{~Hz}, 1 \mathrm{H}), 3.82(\mathrm{dd}, J=4.1,2.8 \mathrm{~Hz}, 1 \mathrm{H}), 4.20$ (d, $J=$ $11.7 \mathrm{~Hz}, 1 \mathrm{H}), 4.43 \mathrm{ppm}(\mathrm{d}, J=11.7 \mathrm{~Hz}, 1 \mathrm{H}) ;{ }^{13} \mathrm{CNMR}(125.7 \mathrm{MHz}$, $\left.\mathrm{CDCl}_{3}\right): \mathrm{d}=21.7\left(\mathrm{CH}_{3}\right), 21.86\left(\mathrm{CH}_{3}\right), 21.9\left(\mathrm{CH}_{3}\right), 54.5(\mathrm{CH}), 58.5\left(\mathrm{CH}_{3}\right)$, $58.7\left(\mathrm{CH}_{3}\right), 60.7\left(\mathrm{CH}_{3}\right), 67.4\left(\mathrm{CH}_{2}\right), 83.2(\mathrm{CH}), 83.8(\mathrm{CH}), 83.9 \mathrm{ppm}$ $(\mathrm{CH})$, (quaternary carbons not observed); IR (film): $\tilde{n^{2}}=2922,1766,1742$, $1731 \mathrm{~cm}^{-1}$; MS $\left(\mathrm{ESI}^{+}\right): \mathrm{m} / \mathrm{z}(\%): 383(100)\left[\mathrm{M}^{+}+\mathrm{Na}\right] ; \operatorname{HRMS}\left(\mathrm{ESI}^{+}\right): \mathrm{m} /$ $Z$ calcd for $\mathrm{C}_{16} \mathrm{H}_{24} \mathrm{NaO}_{9}$ : $383.1318\left[M^{+}+\mathrm{Na}\right]$; found: 383.1322; elemental analysis calcd (\%) for $\mathrm{C}_{16} \mathrm{H}_{24} \mathrm{O}_{9}(360.36)$ : $\mathrm{C} 53.33, \mathrm{H}$ 6.71; found: $\mathrm{C} 53.43, \mathrm{H}$ 6.46 .

Compound 66: colorless oil. $[a]_{\mathrm{D}}=+25 \mathrm{~cm}^{3} \mathrm{~g}^{-1} \mathrm{dm}^{-1}\left(c=0.56\right.$ in $\left.\mathrm{CHCl}_{3}\right)$; ${ }^{1} \mathrm{H} \mathrm{NMR}\left(500 \mathrm{M} \mathrm{Hz} \mathrm{CDCl}_{3}\right): \mathrm{d}=1.54(\mathrm{~s}, 3 \mathrm{H}), 2.05(\mathrm{~s}, 3 \mathrm{H}), 2.09(\mathrm{~s}, 3 \mathrm{H})$, $3.26(\mathrm{~d}, J=9.1 \mathrm{~Hz}, 1 \mathrm{H}), 3.47(\mathrm{~s}, 3 \mathrm{H}), 3.48(\mathrm{~s}, 3 \mathrm{H}), 3.56(\mathrm{~s}, 3 \mathrm{H}), 3.68(\mathrm{dd}$, $J=6.9,4.1 \mathrm{~Hz}, 1 \mathrm{H}), 3.82(\mathrm{~d}, J=3.8 \mathrm{~Hz}, 1 \mathrm{H}), 4.04(\mathrm{dd}, J=9.1,6.9 \mathrm{~Hz}$, $1 \mathrm{H}), 4.18(\mathrm{~d}, J=11.7 \mathrm{~Hz}, 1 \mathrm{H}), 4.69 \mathrm{ppm}(\mathrm{d}, J=11.7 \mathrm{~Hz}, 1 \mathrm{H}) ;{ }^{13} \mathrm{C}$ N M R $\left(125.7 \mathrm{MHz}, \mathrm{CDCl}_{3}\right): \mathrm{d}=20.8\left(\mathrm{CH}_{3}\right), 20.9\left(\mathrm{CH}_{3}\right), 21.7\left(\mathrm{CH}_{3}\right), 50.4(\mathrm{CH})$, $58.8\left(\mathrm{CH}_{3}\right), 59.2\left(\mathrm{CH}_{3}\right), 60.6\left(\mathrm{CH}_{3}\right), 64.1\left(\mathrm{CH}_{2}\right), 83.1(\mathrm{CH}), 83.2(\mathrm{CH})$, $85.2(\mathrm{CH}), 89.6(\mathrm{C}), 101.7(\mathrm{C}), 169.8(\mathrm{C}), 170.4(\mathrm{C}), 205.0 \mathrm{ppm}(\mathrm{C})$; I R (film): $\tilde{n}=2938,1768,1744 \mathrm{~cm}^{-1}$; MS $\left(\mathrm{ESI}^{+}\right): \mathrm{m} / \mathrm{z}(\%): 383(100)\left[\mathrm{M}^{+}\right.$ $+\mathrm{Na}]$; HRMS (ESI ${ }^{+}$): $\mathrm{m} / \mathrm{z}$ calcd for $\mathrm{C}_{16} \mathrm{H}_{24} \mathrm{NaO}_{9}: 383.1318\left[M^{+}+\mathrm{Na}\right]$; found: 383.1324; elemental analysis calcd (\%) for $\mathrm{C}_{16} \mathrm{H}_{24} \mathrm{O}_{9}$ (360.36): C 53.33, H 6.71; found: C 53.35, H 6.68 .

4,7-Anhydro-8-O-tert-butyldiphenylsilyl-1-deoxy-5,6-O-isopropylidene-daltro-octo-2,3-diulosa (70): Prepared from 67 (27 mg, $0.057 \mathrm{mmol}$ ) following the general procedure (Method $\mathrm{B}$ ) using $\mathrm{NaIO}_{4}(37 \mathrm{mg}, 0.171 \mathrm{mmol}$ ) and $\mathrm{RuO}_{2} \times \mathrm{H}_{2} \mathrm{O}(0.3 \mathrm{mg})$. The reaction mixture was stirred at room temperature in the dark for $50 \mathrm{~min}$. The residue was purified by rapid silica gel column chromatography (hexanes/EtOAc $97: 3)$ to give $70(19 \mathrm{mg}$, $0.039 \mathrm{mmol}, 69 \%)$ as a yellow oil and the known lactone $73^{[47]}(2 \mathrm{mg}$, $0.005 \mathrm{mmol}, 6 \%$ )

Compound 70: $[\mathrm{a}]_{\mathrm{D}}=-4 \mathrm{~cm}^{3} \mathrm{~g}^{-1} \mathrm{dm}^{-1}\left(c=1.01\right.$ in $\left.\mathrm{CHCl}_{3}\right) ;{ }^{1} \mathrm{HNMR}$

$\left(500 \mathrm{MHz}, \mathrm{CDCl}_{3}\right): \mathrm{d}=1.09(\mathrm{~s}, 9 \mathrm{H}), 1.27(\mathrm{~s}, 3 \mathrm{H}), 1.36(\mathrm{~s}, 3 \mathrm{H}), 2.37(\mathrm{~s}$, $3 \mathrm{H}$ ), 3.70 (dd, $J=11.4,2.5 \mathrm{~Hz}, 1 \mathrm{H}$ ), 3.88 (dd, $J=11.4,3.5 \mathrm{~Hz}, 1 \mathrm{H}), 4.35$ (dd, $J=2.8,2.8 \mathrm{~Hz}, 1 \mathrm{H}), 4.82(\mathrm{~d}, J=6.0 \mathrm{~Hz}, 1 \mathrm{H}), 5.31(\mathrm{dd}, J=5.7,5.7 \mathrm{~Hz}$, $1 \mathrm{H}), \quad 5.57$ (d, $J=5.4 \mathrm{~Hz}, 1 \mathrm{H}), 7.38-7.65 \mathrm{ppm}(\mathrm{m}, 10 \mathrm{H}) ;{ }^{1} \mathrm{H}$ NMR $\left(500 \mathrm{MHz}, \mathrm{C}_{6} \mathrm{D}_{6}\right): \mathrm{d}=1.08(\mathrm{~s}, 3 \mathrm{H}), 1.13(\mathrm{~s}, 9 \mathrm{H}), 1.35(\mathrm{~s}, 3 \mathrm{H}), 1.99(\mathrm{~s}, 3 \mathrm{H})$, 3.39 (dd, $J=11.0,3.2 \mathrm{~Hz}, 1 \mathrm{H}$ ), 3.66 (dd, $J=11.4,3.5 \mathrm{~Hz}, 1 \mathrm{H}$ ), 4.31 (dd, 
$J=3.5,2.8 \mathrm{~Hz}, 1 \mathrm{H}), 4.65(\mathrm{~d}, J=6.2,1.1 \mathrm{~Hz}, 1 \mathrm{H}), 5.31(\mathrm{dd}, J=5.7,5.7 \mathrm{~Hz}$, $1 \mathrm{H}), \quad 5.66(\mathrm{~d}, J=5.4 \mathrm{~Hz}, 1 \mathrm{H}), \quad 7.19-7.70 \mathrm{ppm}(\mathrm{m}, 10 \mathrm{H}) ;{ }^{13} \mathrm{C} \mathrm{NMR}$ $\left(100.4 \mathrm{MHz}, \mathrm{CDCl}_{3}\right): \mathrm{d}=19.1(\mathrm{C}), 23.6\left(\mathrm{CH}_{3}\right), 24.8\left(\mathrm{CH}_{3}\right), 25.7\left(\mathrm{CH}_{3}\right)$ $26.9\left(3 \times \mathrm{CH}_{3}\right), 65.9\left(\mathrm{CH}_{2}\right), 82.7(\mathrm{CH}), 83.1(\mathrm{CH}), 84.7(\mathrm{CH}), 85.4(\mathrm{CH})$, $113.3(\mathrm{C}), 127.9(4 \times \mathrm{CH}), 129.9(\mathrm{CH}), 130.0(\mathrm{CH}), 132.5(\mathrm{C}), 132.6(\mathrm{C})$ $135.5(2 \times \mathrm{CH}), 135.6(2 \times \mathrm{CH}), 192.2(\mathrm{C}), 197.7 \mathrm{ppm}(\mathrm{C}) ;{ }^{13} \mathrm{CNMR}$ $\left(100.4 \mathrm{MHz}, \mathrm{C}_{6} \mathrm{D}_{6}\right): \mathrm{d}=19.2(\mathrm{C}), 23.1\left(\mathrm{CH}_{3}\right), 24.9\left(\mathrm{CH}_{3}\right), 26.0\left(\mathrm{CH}_{3}\right), 27.0$ $\left(3 \times \mathrm{CH}_{3}\right), 65.9\left(\mathrm{CH}_{2}\right), 83.2(\mathrm{CH}), 83.5(\mathrm{CH}), 84.9(\mathrm{CH}), 85.2(\mathrm{CH}), 113.5$ (C), $128.18(2 \times \mathrm{CH}), 128.21(2 \times \mathrm{CH}), 130.18,(\mathrm{CH}), 130.20(\mathrm{CH}), 133.2$ (C), 133.3 (C), $135.9(2 \times \mathrm{CH}), 136.0(2 \times \mathrm{CH}), 191.7$ (C), 197.4 ppm (C); IR $\left(\mathrm{CHCl}_{3}, 0.2 \mathrm{~mm}\right): \tilde{n}=2936,1717 \mathrm{~cm}^{-1} ; \mathrm{MS}\left(\mathrm{ESI}^{+}\right): \mathrm{m} / z(\%): 537(100)$ $\left[\mathrm{M}^{+}+\mathrm{MeOH}+\mathrm{Na}\right], 505$ (72) $\left[\mathrm{M}^{+}+\mathrm{Na}\right] ; \mathrm{HRMS}\left(\mathrm{ESI}^{+}\right): \mathrm{m} / \mathrm{z}$ calcd for $\mathrm{C}_{28} \mathrm{H}_{38} \mathrm{O}_{7} \mathrm{NaSi}$ : 537.2285 [ $M^{+}+\mathrm{MeOH}+\mathrm{Na}$ ]; found: 537.2288; calcd for $\mathrm{C}_{27} \mathrm{H}_{34} \mathrm{NaO}_{6} \mathrm{Si}\left[M^{+}+\mathrm{Na}\right]$ : 505.2022; found: 505.2025; elemental analysis calcd (\%) for $\mathrm{C}_{27} \mathrm{H}_{34} \mathrm{O}_{6}$ Si (482.64): C 67.19, H 7.10; found: $\mathrm{C}$ 67.32, $\mathrm{H} \quad 7.11$.

Photolysis of 70: A deoxygenated solution of diketone 70 (21 mg, $0.044 \mathrm{mmol})$ in dry $\mathrm{C}_{6} \mathrm{D}_{6}(0.6 \mathrm{~mL})$, was placed in a resonance tube and irradiated with a daylight lamp at $30 \mathrm{8C}$ for $2 \mathrm{~h}$. The reaction was moni- tored by ${ }^{1} \mathrm{H}$ NMR spectroscopy, leading to exclusive formation of the photoenol mixture 74 a (4:1). The mixture was concentrated under re- duced pressure and the residue purified by silica gel column chromatog- raphy (hexanes/EtOAc 9:1) to give known lactone $73(17 \mathrm{mg}$, $0.040 \mathrm{mmol}, 65 \%$ ) from the oxidation of the photoenol by oxygen.

Compound $74 \mathrm{a}:{ }^{1} \mathrm{H} \mathrm{NMR}\left(500 \mathrm{MHz}, \mathrm{C} \mathrm{D}_{6}\right.$;): $\mathrm{d}$ (major isomer) $=0.99(\mathrm{~s}$, $9 \mathrm{H}), 1.23(\mathrm{~s}, 3 \mathrm{H}), 1.36(\mathrm{~s}, 3 \mathrm{H}), 2.46(\mathrm{~s}, 3 \mathrm{H}), 3.22(\mathrm{dd}, J=11.3,2.2 \mathrm{~Hz}$, $1 \mathrm{H}), 3.58(\mathrm{dd}, J=11.4,2.5 \mathrm{~Hz}, 1 \mathrm{H}), 4.39(\mathrm{~m}, 1 \mathrm{H}), 4.68(\mathrm{~d}, J=6.3 \mathrm{~Hz}$, $1 \mathrm{H}), 5.62$ (d, $J=6.3 \mathrm{~Hz}, 1 \mathrm{H}), 6.42$ (br s, $1 \mathrm{H}), 7.18-7.68 \mathrm{ppm}(\mathrm{m}, 10 \mathrm{H})$; d $($ minor isomer $)=1.00(\mathrm{~s}, 9 \mathrm{H}), 1.27(\mathrm{~s}, 3 \mathrm{H}), 1.46(\mathrm{~s}, 3 \mathrm{H}), 2.37(\mathrm{~s}, 3 \mathrm{H})$, 3.26 (dd, $J=11.4,2.5 \mathrm{~Hz}, 1 \mathrm{H}$ ), 3.41 (dd, $J=11.4,2.5 \mathrm{~Hz}, 1 \mathrm{H}), 4.39$ (m, $1 \mathrm{H}), 4.60(\mathrm{~d}, J=6.2 \mathrm{~Hz}, 1 \mathrm{H}), 5.76(\mathrm{~d}, J=6.0 \mathrm{~Hz}, 1 \mathrm{H}), 6.06(\mathrm{br} \mathrm{s}, 1 \mathrm{H})$, 7.18-7.68 ppm (m, 10 H); ${ }^{13} \mathrm{C} \mathrm{NMR}(125.7 \mathrm{M} \mathrm{Hz}, \mathrm{CDCl}$;): d (major isomer $)=19.1(\mathrm{C}), 25.9\left(\mathrm{CH}_{3}\right), 26.1\left(\mathrm{CH}_{3}\right), 26.74\left(3 \times \mathrm{CH}_{3}\right), 26.9\left(\mathrm{CH}_{3}\right)$, $65.0\left(\mathrm{CH}_{2}\right), 80.6(\mathrm{CH}), 81.8(\mathrm{CH}), 87.3(\mathrm{CH}), 112.7(\mathrm{C}), 130.4-136.0(10 \times$ $\mathrm{CH}$ ), 132.5 (C), 133.1 (C), 133.2 (C), 152.2 (C), 191.9 ppm (C); d (minor isomer $)=19.1(\mathrm{C}), 25.6(\mathrm{CH})_{3} 25.7(\mathrm{CH}), 26.7(3 \times \mathrm{CH}), 26.8(\mathrm{CH})$, 65.1( (CH 2, 80.8 (CH), 82.0 (CH), 89.1 (CH), $112.9(\mathrm{C}), 130.35-135.99$ $(10 \times \mathrm{CH}), 132.5(\mathrm{C}), 133.0(\mathrm{C}), 133.2(\mathrm{C}), 152.3(\mathrm{C}), 192.2 \mathrm{ppm}(\mathrm{C})$.

4,7-Anhydro-8-O-tert-butyldiphenylsilyl-1-deoxy-5,6-O-isopropylidene-dallo-octo-2,3-diulose (71): Prepared from 68 (47 mg, $0.102 \mathrm{mmol}$ ) following the general procedure (Method B) using NaIO (73 mg, $0.340 \mathrm{mmol}$ ) and $\mathrm{RuO}_{2} \cdot x \mathrm{H}_{2} \mathrm{O}(0.6 \mathrm{mg})$. The reaction mixture was stirred at room temperature in the dark for $2 \mathrm{~h}$. The residue was purified by rapid silica gel column chromatography (hexanes/EtOAc 97:3) to give 71 as a yellow oil (33 mg, $0.069 \mathrm{mmol}, 68 \%$ ) and the known lactone $73(3 \mathrm{mg}, 0.007 \mathrm{mmol}$,

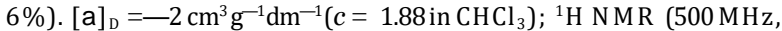
$\mathrm{C}_{6} \mathrm{D}_{6}, 308 \mathrm{C}$; conformer $\left.\mathrm{A}\right): \mathrm{d}=1.17(\mathrm{~s}, 9 \mathrm{H}), 1.18(\mathrm{~s}, 3 \mathrm{H}), 1.47(\mathrm{~s}, 3 \mathrm{H})$, $1.82(\mathrm{~s}, 3 \mathrm{H}), 3.72(\mathrm{~m}, 2 \mathrm{H}), 4.24(\mathrm{dd}, J=9.8,5.0 \mathrm{~Hz}, 1 \mathrm{H}), 4.46(\mathrm{~d}, J=$ $2.5 \mathrm{~Hz}, 1 \mathrm{H}$ ) , 4.67 (dd, $J=6.3,4.7 \mathrm{~Hz}, 1 \mathrm{H}$ ), 5.00 (dd, $J=6.6,3.5 \mathrm{~Hz}, 1 \mathrm{H}$ ), 7.21-7.78 (m, 10H); conformer B: d = $1.13(\mathrm{~s}, 9 \mathrm{H}), 1.17(\mathrm{~s}, 3 \mathrm{H}), 1.46(\mathrm{~s}$, $3 \mathrm{H}), 2.04(\mathrm{~s}, 3 \mathrm{H}), 3.74(\mathrm{dd}, J=11.0,5.4 \mathrm{~Hz}, 1 \mathrm{H}), 3.81(\mathrm{dd}, J=11.0$, $4.7 \mathrm{~Hz}, 1 \mathrm{H}), 4.33(\mathrm{~m}, 1 \mathrm{H}), 4.63(\mathrm{dd}, J=6.3,3.2 \mathrm{~Hz}, 1 \mathrm{H}), 4.86$ (dd, $J=6.3$, $3.5 \mathrm{~Hz}, 1 \mathrm{H}), 5.18(\mathrm{~d}, J=3.5 \mathrm{~Hz}, 1 \mathrm{H}), 7.21-7.78 \mathrm{ppm}(\mathrm{m}, 10 \mathrm{H}) ;{ }^{1} \mathrm{H} \mathrm{NMR}$ $(500 \mathrm{M} \mathrm{Hz}, \mathrm{C} \mathrm{D}, 708 \mathrm{C}): \mathrm{d}=1.14(\mathrm{~s}, 9 \mathrm{H}), 1.19(\mathrm{~s}, 3 \mathrm{H}), 1.45(\mathrm{~s}, 3 \mathrm{H}), 1.86$ $(\mathrm{s}, 3 \mathrm{H}), 3.74(\mathrm{dd}, J=11.0,5.4 \mathrm{~Hz}, 1 \mathrm{H}), 3.78(\mathrm{dd}, J=11.0,5.1 \mathrm{~Hz}, 1 \mathrm{H}$ ), $4.30(\mathrm{~m}, 1 \mathrm{H}), 4.65$ (dd, $J=6.3,3.2 \mathrm{~Hz}, 1 \mathrm{H}), 4.86(\mathrm{dd}, J=6.3,3.5 \mathrm{~Hz}, 1 \mathrm{H}$ ), $5.10(\mathrm{~d}, J=3.5 \mathrm{~Hz}, 1 \mathrm{H}), 7.22-7.76 \mathrm{ppm}(\mathrm{m}, 10 \mathrm{H}) ;{ }^{13} \mathrm{C}\left(100.6 \mathrm{MHz}, \mathrm{C}_{6} \mathrm{D}_{6}\right.$, 308C): d (conformer A) = $19.4(\mathrm{C}), 24.0\left(\mathrm{CH}_{3}\right), 25.5\left(\mathrm{CH}_{3}\right), 27.03\left(\mathrm{CH}_{3}\right)$ $27.4\left(3 \times \mathrm{CH}_{3}\right), 64.4\left(\mathrm{CH}_{2}\right), 82.4(\mathrm{CH}), 82.7(\mathrm{CH}), 85.7(\mathrm{CH}), 86.5(\mathrm{CH})$, 114.1 (C), $128.1-136.0(10 \times \mathrm{CH}), \quad 133.57-133.64(2 \times \mathrm{C}), \quad 195.3(\mathrm{C})$, 197.3 ppm (C); d (conformer B) $=19.4(\mathrm{C}), 23.1\left(\mathrm{CH}_{3}\right), 25.4\left(\mathrm{CH}_{3}\right), 27.0$ $\left(\mathrm{CH}_{3}\right), 27.6\left(3 \times \mathrm{CH}_{3}\right), 64.7\left(\mathrm{CH}_{2}\right), 81.6(\mathrm{CH}), 82.0(\mathrm{CH}), 86.3(\mathrm{CH}), 86.9$ $(\mathrm{CH}), 114.1(\mathrm{C}), 128.1-136.0(10 \times \mathrm{CH}), 133.67-133.64(2 \times \mathrm{C}), 195.3(\mathrm{C})$, 197.3 ppm (C); ${ }^{13} \mathrm{C}$ N MR $\left(125.7 \mathrm{MHz}, \mathrm{C}_{6} \mathrm{D}_{6}, 708 \mathrm{C}\right): \mathrm{d}=19.5(\mathrm{C}), 24.0$ $\left(\mathrm{CH}_{3}\right), 25.6\left(\mathrm{CH}_{3}\right), 27.2\left(\mathrm{CH}_{3}\right), 27.4\left(3 \times \mathrm{CH}_{3}\right), 64.6\left(\mathrm{CH}_{2}\right), 82.6(\mathrm{CH}), 82.9$ $(\mathrm{CH}), 85.6(\mathrm{CH}), 86.8(\mathrm{CH}), 114.3(\mathrm{C}), 128.09(2 \times \mathrm{CH}), 128.10(2 \times \mathrm{CH})$, $130.05(\mathrm{CH}), 130.06(\mathrm{CH}), 133.9(\mathrm{C}), 134.0(\mathrm{C}), 136.04(4 \times \mathrm{CH}), 195.7(\mathrm{C})$, 197.5 ppm (C); IR $\left(\mathrm{CHCl}_{3}, 0.2 \mathrm{~mm}\right): \mathrm{n}<\ddot{U}=>=2936,1721 \mathrm{~cm}^{-1}$;
MS (ESI $\left.{ }^{+}\right): m / z(\%): 537(100)\left[M^{+}+\mathrm{MeOH}+\mathrm{Na}\right], 505(38)\left[M^{+}+\mathrm{Na}\right]$; HRMS (ESI $\left.{ }^{+}\right): \quad m / z$ calcd for $\mathrm{C}_{28} \mathrm{H}_{38} \mathrm{O}_{7} \mathrm{NaSi}$ : $537.2285\left[M^{+}\right.$ $+\mathrm{MeOH}+\mathrm{Na}$ ]; found: 537.2293 ; calcd for $\mathrm{C}_{27} \mathrm{H}_{34} \mathrm{NaO}_{6} \mathrm{Si}\left[M^{+}+\mathrm{Na}\right]$ : 505.2022; found: 505.2033; elemental analysis calcd (\%) for $\mathrm{C}_{27} \mathrm{H}_{34} \mathrm{O}_{6} \mathrm{Si}$ (482.64):C 67.19, H 7.10; found: C 67.38, H 7.38.

Photolysis of 71: A deoxygenated solution of diketone 71 (29 mg, $0.061 \mathrm{mmol})$ in dry $\mathrm{C}_{6} \mathrm{D}_{6}(0.6 \mathrm{~mL})$, was placed in a resonance tube and irradiated with a daylight lamp at $308 \mathrm{C}$ for $2 \mathrm{~h}$. The reaction was monitored by ${ }^{1} \mathrm{H}$ NMR spectroscopy, leading to exclusive formation of the photoenol mixture 74 a (3:5). The mixture was concentrated under reduced pressure and the residue purified by silica gel column chromatography (hexanes/EtOAc 9:1) to give the known lactone 73 (12 mg, $0.028 \mathrm{mmol}, 64 \%$ ) from the oxidation of the photoenol by oxygen. 4,7-Anhydro-8-O-tert-butyldiphenylsilyl-1-deoxy-5,6-O-isopropylidene-dtalo-octo-2,3-diulose (72): Prepared from 69 (70 $\mathrm{mg}, 0.160 \mathrm{mmol})$ following the general procedure (Method B) using $\mathrm{NaIO}_{4}(116 \mathrm{mg}, 0.540 \mathrm{mmol}$ ) and $\mathrm{RuO}_{2} \cdot \mathrm{H}_{2} \mathrm{O}(1 \mathrm{mg})$. The reaction mixture was stirred at room temperature in the dark for $3 \mathrm{~h}$. The residue was purified by rapid silica gel column chromatography (hexanes/EtOAc 9:1) to give 72 (30 mg, $0.062 \mathrm{mmol}, 39 \%)$ as a yellow oil. [a] $={ }_{D_{D}} 3 \mathrm{~cm}^{3} \mathrm{~g}^{-1} \mathrm{dm}^{-1}(c=1.14 \mathrm{in}$

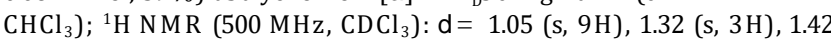
(s, $3 \mathrm{H}), 2.37$ (s, 3H), 3.93 (dd, $J=10.4,6.3 \mathrm{~Hz}, 1 \mathrm{H}), 3.99$ (dd, $J=10.4$, $6.0 \mathrm{~Hz}, 1 \mathrm{H}$ ), 4.08 (ddd, $J=6.0,6.0,3.5 \mathrm{~Hz}, 1 \mathrm{H}$ ), 4.73 (dd, $J=6.0,3.5 \mathrm{~Hz}$, $1 \mathrm{H}), 4.94(\mathrm{dd}, J=6.0,1.3 \mathrm{~Hz}, 1 \mathrm{H}), 5.10(\mathrm{br} \mathrm{s}, 1 \mathrm{H}), 7.33-7.71 \mathrm{ppm}(\mathrm{m}$, $10 \mathrm{H}) ;{ }^{13} \mathrm{CNMR}\left(125.7 \mathrm{MHz}, \mathrm{CDCl}_{3}\right): \mathrm{d}=19.2(\mathrm{C}), 24.5\left(\mathrm{CH}_{3}\right), 25.3$ $\left(\mathrm{CH}_{3}\right), 26.3\left(\mathrm{CH}_{3}\right), 26.8\left(3 \times \mathrm{CH}_{3}\right), 61.8\left(\mathrm{CH}_{2}\right), 80.8(\mathrm{CH}), 82.1(\mathrm{CH}), 83.2$ $(\mathrm{CH}), 84.4(\mathrm{CH}), 113.3(\mathrm{C}), 127.58(2 \times \mathrm{CH}), 127.63(2 \times \mathrm{CH}), 129.6(2 \times$ $\mathrm{CH}), 133.5(\mathrm{C}), 133.6(\mathrm{C}), 135.67(2 \times \mathrm{CH}), 135.71(2 \times \mathrm{CH}), 195.6(\mathrm{C})$, $198.1 \mathrm{ppm}(\mathrm{C}) ;{ }^{1} \mathrm{H} \mathrm{NMR}\left(500 \mathrm{MHz}, \mathrm{C}_{6} \mathrm{D}_{6}\right): \mathrm{d}=1.12(\mathrm{~s}, 3 \mathrm{H}), 1.19(\mathrm{~s}, 9 \mathrm{H})$, $1.32(\mathrm{~s}, 3 \mathrm{H}), 1.80(\mathrm{~s}, 3 \mathrm{H}), 4.14-4.25(\mathrm{~m}, 3 \mathrm{H}), 4.35(\mathrm{dd}, J=6.0,3.5 \mathrm{~Hz}$, $1 \mathrm{H}), 4.69$ (dd, $J=6.0,1.3 \mathrm{~Hz}, 1 \mathrm{H}), 5.16(\mathrm{br} \mathrm{s}, 1 \mathrm{H}), 7.21-7.85(\mathrm{~m}, 10 \mathrm{H})$; ${ }^{13} \mathrm{C} \mathrm{NMR}\left(100.6 \mathrm{MHz}, \mathrm{C}_{6} \mathrm{D}_{6}\right): \mathrm{d}=19.5(\mathrm{C}), 23.7\left(\mathrm{CH}_{3}\right), 25.2\left(\mathrm{CH}_{3}\right), 26.4$ $\left(\mathrm{CH}_{3}\right), 27.0\left(3 \times \mathrm{CH}_{3}\right), 62.5\left(\mathrm{CH}_{2}\right), 81.2(\mathrm{CH}), 82.7(\mathrm{CH}), 83.6(\mathrm{CH}), 84.7$ $(\mathrm{CH}), 113.1(\mathrm{C}), 127.97(2 \times \mathrm{CH}), 128.0(2 \times \mathrm{CH}), 129.93(2 \times \mathrm{CH}), 133.9$ (C), 134.0 (C), $136.06(2 \times \mathrm{CH}), 136.12(2 \times \mathrm{CH}), 195.8$ (C), $197.5 \mathrm{ppm}$ (C); IR $\left(\mathrm{CHCl}_{3}, 0.2 \mathrm{~mm}\right): \tilde{\mathrm{n}}=2933,1719 \mathrm{~cm}^{-1}$; $\mathrm{MS}\left(\mathrm{ESI}^{+}\right): \mathrm{m} / \mathrm{z}(\%): 537$ (100) $\left[\mathrm{M}^{+}+\mathrm{MeOH}+\mathrm{Na}\right] ; 505(39)\left[\mathrm{M}^{+}+\mathrm{Na}\right]$; HRM S $\left(\mathrm{ESI}^{+}\right)$: $\mathrm{m} / \mathrm{zcalcd}$ for $\mathrm{C}_{28} \mathrm{H}_{38} \mathrm{NaO}_{7} \mathrm{Si}\left[M^{+}+\mathrm{MeOH}+\mathrm{Na}\right]: 537.2285$; found: 537.2287; calcd for $\mathrm{C}_{27} \mathrm{H}_{34} \mathrm{NaO}_{6} \mathrm{Si}\left[M^{+}+\mathrm{Na}\right]$ : 505.2022; found: 505.2027; elemental analysis calcd (\%) for $\mathrm{C}_{27} \mathrm{H}_{34} \mathrm{O}_{6} \mathrm{Si}$ (482.64): C 67.19, $\mathrm{H} \mathrm{7.10;} \mathrm{found:} \mathrm{C} 66.80, \mathrm{H}$ 7.51.

Photolysis of 72: A deoxygenated solution of diketone $72(42.7 \mathrm{mg}$, $0.09 \mathrm{mmol}$ ) in purified $\mathrm{CDCl}_{3}(1 \mathrm{~mL})$, was placed in a resonance tube and irradiated with a daylight lamp at $308 \mathrm{C}$ for $9 \mathrm{~h}$. The reaction was monitored by ${ }^{1} \mathrm{H}$ NMR spectroscopy, leading to exclusive formation of 75 besides a small amount of photoenol intermediate $74 \mathrm{~b}$. The mixture was kept at room temperature in the dark for $14 \mathrm{~h}$ for the complete transfor- mation of $74 \mathrm{~b}$ into 75 . The solution could be concentrated at low temper- ature under reduced pressure to give $75(41.4 \mathrm{mg})$ as a hemiketal mixture $(10: 8)$. Compound 75 was not stable enough to withstand chromatograph- ic purification (silica gel or alumina). Notwithstanding, the crude residue (colorless oil) was pure enough to allow the complete analytical and spectroscopic characterization.

Photoenol $74 \mathrm{~b}:{ }^{1} \mathrm{H} \mathrm{N}$ MR $\left(400 \mathrm{MHz}, \mathrm{CDCl}_{3}\right): \mathrm{d}=2.25(\mathrm{~s}, 3 \mathrm{H}), 4.26(\mathrm{~d}, J=$ $18.8 \mathrm{~Hz}, 1 \mathrm{H}$ ), 4.39 (d, $J=18.5 \mathrm{~Hz}, 1 \mathrm{H}), 4.96$ (d, $J=7.4 \mathrm{~Hz}, 1 \mathrm{H}$ ), 5.37 (dd, $J=8.5,1.3 \mathrm{~Hz}, 1 \mathrm{H}), 5.51(\mathrm{dd}, J=8.7,7.4 \mathrm{~Hz}, 1 \mathrm{H}), 6.71(\mathrm{br} \mathrm{d}, J=1.6 \mathrm{~Hz}$, $1 \mathrm{H})$.

Compound 75: ${ }^{1} \mathrm{H}\left(400 \mathrm{MHz}, \mathrm{CDCl}_{3}\right.$; major isomer): $\mathrm{d}=1.11(\mathrm{~s}, 9 \mathrm{H}), 1.33$ $(\mathrm{s}, 3 \mathrm{H}), 1.43(\mathrm{~s}, 3 \mathrm{H}), 2.30(\mathrm{~s}, 3 \mathrm{H}), 3.90(\mathrm{~d}, J=10.6 \mathrm{~Hz}, 1 \mathrm{H}), 4.02(\mathrm{~d}$, $J=10.6 \mathrm{~Hz}, 1 \mathrm{H}), 4.17(\mathrm{dd}, J=5.8,1.3 \mathrm{~Hz}, 1 \mathrm{H}), 4.87(\mathrm{dd}, J=5.8,3.2 \mathrm{~Hz}$, $1 \mathrm{H}$ ), $5.95(\mathrm{dd}, J=3.3,1.2 \mathrm{~Hz}, 1 \mathrm{H}), 7.35-7.76 \mathrm{ppm}(\mathrm{m}, 10 \mathrm{H})$; d (minor isomer $)=1.08(\mathrm{~s}, 9 \mathrm{H}), 1.33(\mathrm{~s}, 3 \mathrm{H}), 1.43(\mathrm{~s}, 3 \mathrm{H}), 2.31(\mathrm{~s}, 3 \mathrm{H}), 3.85(\mathrm{~s}$, $2 \mathrm{H}), 4.30(\mathrm{~d}, J=6.6 \mathrm{~Hz}, 1 \mathrm{H}), 4.70(\mathrm{dd}, J=6.4,4.0 \mathrm{~Hz}, 1 \mathrm{H}), 6.16(\mathrm{~d}, J=$ $4.0 \mathrm{~Hz}, 1 \mathrm{H}), 7.35-7.76 \mathrm{ppm}(\mathrm{m}, 10 \mathrm{H}) ;{ }^{13} \mathrm{C} \mathrm{NMR}\left(100.6 \mathrm{MHz} \mathrm{CDCl}_{3}\right): \mathrm{d}$ (major isomer) $=19.2(\mathrm{C}), 25.9\left(\mathrm{CH}_{3}\right), 26.5\left(\mathrm{CH}_{3}\right), 26.7\left(\mathrm{CH}_{3}\right), 26.80(3 \times$ $\left.\mathrm{CH}_{3}\right), 66.8\left(\mathrm{CH}_{2}\right), 69.3(\mathrm{CH}), 73.3(\mathrm{CH}), 98.0(\mathrm{C}), 107.6(\mathrm{CH}), 110.8(\mathrm{C})$, 127.6-135.7 (10× CH), 132.4 (C), 132.5 (C), 146.7 (C), $194.61 \mathrm{ppm}(\mathrm{C})$; d $($ minor isomer $)=19.3(\mathrm{C}), 25.5\left(\mathrm{CH}_{3}\right), 26.78\left(3 \times \mathrm{CH}_{3}\right), 65.7\left(\mathrm{CH}_{2}\right), 68.7$ 
(CH), $72.1(\mathrm{CH}), 97.2(\mathrm{C}), 105.1(\mathrm{CH}), 109.7(\mathrm{C}), 127.6-135.7(10 \times \mathrm{CH})$, 132.6 (C), 132.7 (C), 149.0 (C), $194.57 \mathrm{ppm}$ (C) (isopropylidene methyl atoms not observed); UV/Vis $\left(\mathrm{CH}_{2} \mathrm{Cl}_{2}\right): \mathrm{I}_{\max }(\mathrm{e})=262 \mathrm{~nm}(\mathrm{sh})$ $\left(2253 \mathrm{~mol}^{-1} \mathrm{~m}^{3} \mathrm{~cm}^{-1}\right), \quad 259 \mathrm{~nm} \quad\left(2374 \mathrm{~mol}^{-1} \mathrm{~m}^{3} \mathrm{~cm}^{-1}\right), \quad 256 \mathrm{~nm} \quad$ (sh) (2274 mol-1 $\left.\mathrm{m}^{3} \mathrm{~cm}^{-1}\right), 228 \mathrm{~nm}\left(5844 \mathrm{~mol}^{-1} \mathrm{~m}^{3} \mathrm{~cm}^{-1}\right)$; IR $\left(\mathrm{CHCl}_{3}, 0.2 \mathrm{~mm}\right):$ ก

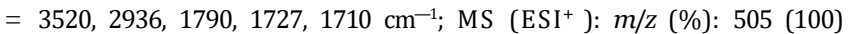
$\left[M^{+}+\mathrm{Na}\right]$; HRMS $\left(\mathrm{ESI}^{+}\right.$): $\mathrm{m} / \mathrm{zcalcd}$ for $\mathrm{C}_{27} \mathrm{H}_{34} \mathrm{NaO}_{6} \mathrm{Si}: 505.2022\left[\mathrm{M}^{+}\right.$ $+\mathrm{Na}$ ]; found: 505.2019; elemental analysis calcd (\%) for $\mathrm{C}_{27} \mathrm{H}_{34} \mathrm{O}_{6} \mathrm{Si}$ (482.64): C 67.19, H 7.10; found: C 67.19, H 6.98.

Methyl (1S,2R,3S,4R,5S)-2-acetyloxy-3,4,5-trimethoxy-2-(methoxymethyl)cyclopentane-1-carboxylate (76): $\mathrm{H}_{5} \mathrm{IO}_{6}(23 \mathrm{mg}, 0.101 \mathrm{mmol})$ was added to a solution of $2(17 \mathrm{mg}, 0.059 \mathrm{mmol})$ in $\mathrm{MeOH}(3.5 \mathrm{~mL})$. The mixture was stirred for $2 \mathrm{~h}$ at room temperature and concentrated under reduced pressure. A solution of the residue in $\mathrm{Et}_{2} \mathrm{O}$ was treated with diazomethane at $0 \mathrm{8C}$. The mixture was then concentrated under reduced pressure and the residue purified by silica gel column chromatography (hexanes/EtOAc 7:3) to give $76(16 \mathrm{mg}, 0.05 \mathrm{mmol}, 85 \%)$ as a colorless oil: $[\mathrm{a}]_{\mathrm{D}}=+32 \mathrm{~cm}^{3} \mathrm{~g}^{-1} \mathrm{dm}^{-1}\left(\mathrm{c}=1.35\right.$ in $\left.\mathrm{CHCl}_{3}\right) ;{ }^{1} \mathrm{H} \mathrm{NMR}(500 \mathrm{MHz}$, $\left.\mathrm{CDCl}_{3}\right): \mathrm{d}=1.99(\mathrm{~s}, 3 \mathrm{H}), 3.13(\mathrm{~d}, J=8.8 \mathrm{~Hz}, 1 \mathrm{H}), 3.35(\mathrm{~s}, 3 \mathrm{H}), 3.42(\mathrm{~s}$, $3 \mathrm{H}), 3.46(\mathrm{~s}, 3 \mathrm{H}), 3.48(\mathrm{~s}, 3 \mathrm{H}), 3.51(\mathrm{dd}, J=5.7,5.0 \mathrm{~Hz}, 1 \mathrm{H}), 3.70(\mathrm{~s}$, $3 \mathrm{H}), 3.77(\mathrm{~d}, J=9.5 \mathrm{~Hz}, 1 \mathrm{H}), 3.89(\mathrm{brd}, J=5.1 \mathrm{~Hz}, 1 \mathrm{H}), 3.94(\mathrm{~d}, J=$ $9.8 \mathrm{~Hz}, 1 \mathrm{H}$ ), $4.09 \mathrm{ppm}$ (ddd, $J=8.8,5.7,0.6 \mathrm{~Hz}, 1 \mathrm{H}) ;{ }^{13} \mathrm{C} \mathrm{NMR}$ $\left(100.6 \mathrm{MHz}, \mathrm{CDCl}_{3}\right): \mathrm{d}=21.7\left(\mathrm{CH}_{3}\right), 51.9(\mathrm{CH}), 52.2\left(\mathrm{CH}_{3}\right), 58.0\left(\mathrm{CH}_{3}\right)$, $58.3\left(\mathrm{CH}_{3}\right), 58.9\left(\mathrm{CH}_{3}\right), 59.3\left(\mathrm{CH}_{3}\right), 70.5\left(\mathrm{CH}_{2}\right), 84.7(\mathrm{CH}), 86.8(\mathrm{CH})$, $86.9(\mathrm{C}), 88.6(\mathrm{CH}), 169.8(\mathrm{C}), 170.7 \mathrm{ppm}(\mathrm{C})$; IR (film): $\tilde{n}=3013,2936$ $1740 \mathrm{~cm}^{-1}$; MS (EI): $m / z(\%): 321(<1)\left[M^{+}+\mathrm{H}\right], 260(2) ; H R M S(E I):$ $\mathrm{m} / \mathrm{z}$ calcd for $\mathrm{C}_{14} \mathrm{Q}_{8}: 321.1549$; found $\left[M^{+}+\mathrm{H}\right]: 321.1558$; elemental analysis calcd (\%) for $\mathrm{C}_{14} \mathrm{H}_{24} \mathrm{O}_{8}$ (320.33): C 52.49, $\mathrm{H}$ 7.55; found: $\mathrm{C} 52.27$ H 7.49.

Methyl (1S,2R,3S,4R,5S)-2-acetyloxy-3,4,5-tris(benzyloxy)-2-(benzyloxymethyl)cyclopentane-1-carboxylate (77): $\mathrm{H}_{5} \mathrm{IO}_{6}(12 \mathrm{mg}, 0.054 \mathrm{mmol})$ was added to a solution of $25(16 \mathrm{mg}, 0.027 \mathrm{mmol})$ in $\mathrm{MeOH}(1.6 \mathrm{~mL})$. The mixture was stirred for $1.5 \mathrm{~h}$ at room temperature and concentrated under reduced pressure. A solution of the residue in $\mathrm{Et}_{2} \mathrm{O}(0.5 \mathrm{~mL})$ was treated with diazomethane at 0 8C. The mixture was then concentrated under reduced pressure and the residue purified by silica gel column chromatography (hexanes/EtOAc 90:10) to give 77 (11 mg, 0.018mmol, $65 \%)$ as a colorless oil: [a] $=\hbar_{\mathrm{D}} 14 \mathrm{~cm}^{3} \mathrm{~g}^{-1} \mathrm{dm}^{-1}(c=0.52 \mathrm{in} \mathrm{CHCl})$; ${ }^{1} \mathrm{H} \mathrm{NMR}\left(500 \mathrm{MHz}, \mathrm{CDCl}_{3}\right): \mathrm{d}=1.95(\mathrm{~s}, 3 \mathrm{H}), 3.37(\mathrm{~d}, J=8.8 \mathrm{~Hz}, 1 \mathrm{H})$, 3.63 (s, $3 \mathrm{H}$ ), 3.94 (d, $J=9.5 \mathrm{~Hz}, 1 \mathrm{H}$ ), 3.96 (dd, $J=5.5,5.5 \mathrm{~Hz}, 1 \mathrm{H}$ ), 4.12 (d, $J=9.8 \mathrm{~Hz}, 1 \mathrm{H}), 4.29(\mathrm{brd}, J=5.1 \mathrm{~Hz}, 1 \mathrm{H}), 4.48(\mathrm{dd}, J=8.8,6.0 \mathrm{~Hz}$, $1 \mathrm{H}), 4.49$ (d, $J=12.6 \mathrm{~Hz}, 1 \mathrm{H}), 4.52(\mathrm{~d}, J=12.0 \mathrm{~Hz}, 1 \mathrm{H}), 4.55(\mathrm{~d}, J=$ $11.7 \mathrm{~Hz}, 1 \mathrm{H}), 4.58(\mathrm{~d}, J=11.7 \mathrm{~Hz}, 1 \mathrm{H}), 4.61(\mathrm{~d}, J=11.7 \mathrm{~Hz}, 1 \mathrm{H}), 4.63(\mathrm{~d}$, $J=11.4 \mathrm{~Hz}, 1 \mathrm{H}), 4.65(\mathrm{~d}, J=11.4 \mathrm{~Hz}, 1 \mathrm{H}), 4.69(\mathrm{~d}, J=11.7 \mathrm{~Hz}, 1 \mathrm{H}), 7.23-$ $7.31 \mathrm{ppm}(\mathrm{m}, 20 \mathrm{H}) ;{ }^{13} \mathrm{C} \mathrm{NMR}\left(125.7 \mathrm{MHz}, \mathrm{CDCl}_{3}\right): \mathrm{d}=21.7\left(\mathrm{CH}_{3}\right)$, $51.9(\mathrm{CH}), 52.7(\mathrm{CH}), 68.7(\mathrm{CH}), 72.4(\mathrm{CH}), 72.8(\mathrm{CH}), 73_{2} 2(\mathrm{CH})$, $73.4(\mathrm{CH}), 83.4(\mathrm{CH}), 85.2(\mathrm{CH}), 87.0(\mathrm{C}), 87.1(\mathrm{CH}), 127.39(\mathrm{CH})$, $127.43(2 \times \mathrm{CH}), 127.55(\mathrm{CH}), 127.58(\mathrm{CH}), 127.66(3 \times \mathrm{CH}), 127.74(2 \times$ $\mathrm{CH}), 127.8(2 \times \mathrm{CH}), 128.2(2 \times \mathrm{CH}), 128.25(2 \times \mathrm{CH}), 128.3(4 \times \mathrm{CH})$, $138.1(\mathrm{C}), 138.2(2 \times \mathrm{C}), 138.3(\mathrm{C}), 169.9(\mathrm{C}), 170.7 \mathrm{ppm}(\mathrm{C})$; IR (film): $\tilde{n}=$ 2928, $1738 \mathrm{~cm}^{-1}$; MS (FAB): $m / z(\%): 647$ (5) [ $\left.M^{+}+\mathrm{Na}\right]$; HRMS (FAB): $\mathrm{m} / \mathrm{Z}$ : calcd for $\mathrm{C}_{38} \mathrm{H}_{40} \mathrm{NaO}_{8}: 647.2621\left[M^{+}+\mathrm{Na}\right]$; found: 647.2651 ; elemental analysis calcd (\%) for $\mathrm{C}_{38} \mathrm{H}_{40} \mathrm{O}_{8}$ (624.72): $\mathrm{C} 73.06, \mathrm{H} \mathrm{6.45}$; found: $\mathrm{C} 72.65$, H 6.82 .

Methyl (1S,2R,3S,4R,5S)-2-acetyloxy-3,4,5-tris(benzyloxy)-2-(tert-butyldiphenylsilyloxymethyl)cyclopentano-1-carboxylate (78): $\mathrm{H}_{5} \mathrm{IO}_{6} \quad(15 \mathrm{mg}$, $0.067 \mathrm{mmol})$ was added to a solution of $26(25 \mathrm{mg}, 0.034 \mathrm{mmol})$ in $\mathrm{MeOH}(2.0 \mathrm{~mL})$. The mixture was stirred for $3 \mathrm{~h}$ at room temperature and concentrated under reduced pressure. A solution of the residue in $\mathrm{Et}_{2} \mathrm{O}(0.8$ $\mathrm{mL}$ ) was treated with diazomethane at 0 8C. The mixture was then concentrated under reduced pressure and the residue purified by silica gel column chromatography (hexanes/EtOAc 90:10) to give 78 (11 mg, 0.014 mmol, $42 \%)$ as a colorless oil: $[\mathrm{a}]_{\mathrm{D}}=+17 \mathrm{~cm}^{3} \mathrm{~g}^{-1} \mathrm{dm}^{-1}(c=0.40$ in $\left.\mathrm{CHCl}_{3}\right) ;{ }^{1} \mathrm{H} \mathrm{NMR}\left(500 \mathrm{MHz}, \mathrm{CDCl}_{3}\right): \mathrm{d}=1.03(\mathrm{~s}, 9 \mathrm{H}), 1.88(\mathrm{~s}$,

$3 \mathrm{H}), 3.610(\mathrm{~s}, 3 \mathrm{H}), 3.611(\mathrm{~d}, J=8.8 \mathrm{~Hz}, 1 \mathrm{H}), 3.93(\mathrm{~d}, J=10.1 \mathrm{~Hz}, 1 \mathrm{H})$, $4.13(\mathrm{dd}, J=7.1,7.1 \mathrm{~Hz}, 1 \mathrm{H}), 4.23(\mathrm{~d}, J=9.8 \mathrm{~Hz}, 1 \mathrm{H}), 4.39(\mathrm{~d}, J=7.3 \mathrm{~Hz}$, $1 \mathrm{H}), 4.52$ (dd, $J=8.8,6.9 \mathrm{~Hz}, 1 \mathrm{H}), 4.62(\mathrm{~d}, J=11.7 \mathrm{~Hz}, 1 \mathrm{H}), 4.65$ (d, $J=$ $12.0 \mathrm{~Hz}, 1 \mathrm{H}$ ), 4.67 (d, $J=12.3 \mathrm{~Hz}, 1 \mathrm{H}), 4.693(\mathrm{~d}, J=11.0 \mathrm{~Hz}, 1 \mathrm{H}), 4.696$ $(\mathrm{d}, J=11.7 \mathrm{~Hz}, 1 \mathrm{H}), 4.74(\mathrm{~d}, J=11.7 \mathrm{~Hz}, 1 \mathrm{H}), 7.19-7.4(\mathrm{~m}, 21 \mathrm{H}), 7.65-$ $7.69 \mathrm{ppm}(\mathrm{m}, 4 \mathrm{H}) ;{ }^{13} \mathrm{C} \mathrm{NMR}\left(125.7 \mathrm{MHz}, \mathrm{CDCl}_{3}\right): \mathrm{d}=19.3(\mathrm{C}), 21.6$ $\left(\mathrm{CH}_{3}\right), 26.8\left(3 \times \mathrm{CH}_{3}\right), 51.6(\mathrm{CH}), 51.8\left(\mathrm{CH}_{3}\right), 62.8\left(\mathrm{CH}_{2}\right), 72.7\left(\mathrm{CH}_{2}\right), 72.8$ $\left(\mathrm{CH}_{2}\right), 73.7\left(\mathrm{CH}_{2}\right), 82.2(\mathrm{CH}), 85.2(\mathrm{CH}), 86.5(\mathrm{C}), 86.7(\mathrm{CH}), 127.4$ $(\mathrm{CH}), 127.5(3 \times \mathrm{CH}), 127.6(\mathrm{CH}), 127.62(4 \times \mathrm{CH}), 127.7(2 \times \mathrm{CH}), 127.9$ $(2 \times \mathrm{CH}), 128.2(2 \times \mathrm{CH}), 128.27(2 \times \mathrm{CH}), 128.31(2 \times \mathrm{CH}), 129.6(\mathrm{CH})$, $129.7(\mathrm{CH}), 135.79(2 \times \mathrm{CH}), 135.8(2 \times \mathrm{CH}), 132.9(\mathrm{C}), 138.2(\mathrm{C}), 138.4$ (C), $138.6(\mathrm{C}), 169.6$ (C), $170.9 \mathrm{ppm}(\mathrm{C})$; IR $\left(\mathrm{CHCl}_{3}, 0.2 \mathrm{~mm}\right): \tilde{n}=2929$, $1745 \mathrm{~cm}^{-1}$; MS (FAB): $m / z(\%): 795$ (10) $\left[M^{+}+\mathrm{Na}\right]$; HRMS (FAB): $\mathrm{m} / \mathrm{z}$ calcd for $\mathrm{C}_{47} \mathrm{H}_{52} \mathrm{NaO}_{8} \mathrm{Si}, 795.3329\left[M^{+}+\mathrm{Na}\right]$; found: 795.3303; elemental analysis calcd for $\mathrm{C}_{47} \mathrm{H}_{52} \mathrm{O}_{8} \mathrm{Si}$ (772.99): C 73.03, $\mathrm{H}$ 6.78; found: $\mathrm{C} 73.16, \mathrm{H}$ 6.99 .

Methyl (1S,2R,3S,5R)-2-(acetyloxy)-3,5-dimethoxy-2-(methoxymethyl)cyclopentane-1-carboxylate (80): MethodA: A solution of 46 (20.0 mg, $0.08 \mathrm{mmol}$ ) in $\mathrm{MeOH}(5 \mathrm{~mL})$ containing $\mathrm{H}_{5} \mathrm{IO}_{6}(35.4 \mathrm{mg}, 0.16 \mathrm{mmol})$ was stirred at room temperature for $5 \mathrm{~h}$. The mixture was concentrated under reduced pressure and the residue purified by silica gel column chromatography (EtO Ac! EtO Ac / MeOH 95:5) to give acid 79 (19.9mg, $0.072 \mathrm{mmol}, 90 \%)$ as a colorless oil. [a $]_{\mathrm{D}}=+16 \mathrm{~cm}^{3} \mathrm{~g}^{-1} \mathrm{dm}^{-1}(c=0.55 \mathrm{in}$ $\left.\mathrm{CHCl}_{3}\right) ;{ }^{1} \mathrm{H} \mathrm{NMR}\left(500 \mathrm{MHz}, \mathrm{CDCl}_{3}\right): \mathrm{d}=1.80$ (ddd, $J=14.2,5.0,5.0 \mathrm{~Hz}$, $1 \mathrm{H}$ ), 2.02 (s, $3 \mathrm{H}$ ), 2.31 (ddd, $J=14.2,7.3,5.6 \mathrm{~Hz}, 1 \mathrm{H}$ ), 3.15 (d, $J=6.3 \mathrm{~Hz}, 1$ H), 3.29 (s, $3 \mathrm{H}), 3.39$ (s, $3 \mathrm{H}$ ), 3.53 (s, $3 \mathrm{H}), 3.89$ (d, $J=10.7 \mathrm{~Hz}, 1 \mathrm{H}), 4.19$ (dd, $J=5.4,5.0 \mathrm{~Hz}, 1 \mathrm{H}), 4.46(\mathrm{~m}, 1 \mathrm{H}), 4.47 \mathrm{ppm}(\mathrm{d}, J=11.0 \mathrm{~Hz}, 1 \mathrm{H}) ;{ }^{13} \mathrm{C}$ NMR (125.7 MHz, CDCl $\left.{ }_{3}\right): \mathrm{d}=21.7\left(\mathrm{CH}_{3}\right), 34.3\left(\mathrm{CH}_{2}\right), 57.6\left(\mathrm{CH}_{3}\right), 57.8$ $\left(\mathrm{CH}_{3}\right), 59.6\left(\mathrm{CH}_{3}\right), 60.0(\mathrm{CH}), 73.4\left(\mathrm{CH}_{2}\right), 80.7(\mathrm{CH}), 84.1(\mathrm{CH}), 89.2(\mathrm{C})$, 169.2 (C), $169.9 \mathrm{ppm}(\mathrm{C})$; IR (film): $\mathrm{n}^{\sim}=3490,2935,1743 \mathrm{~cm}^{-1}$; MS $\left(\mathrm{ESI}^{+}\right.$): m/z (\%): 299 (100) [ $\left.M^{+}+\mathrm{Na}\right]$; HRMS (ESI ${ }^{+}$): $m / z$ calcd for $\mathrm{C}_{12} \mathrm{H}_{20} \mathrm{O}_{7} \mathrm{Na}$ : $299.1107\left[M^{+}+\mathrm{Na}\right.$ ]; found: 299.1112; elemen- tal analysis calcd (\%) for $\mathrm{C}_{12} \mathrm{H}_{20} \mathrm{O}_{7}$ (276.28): C 52.17, $\mathrm{H}$ 7.30; found: C 52.12, $\mathrm{H}$ 7.39.

Method $A$ and subsequent diazomethane esterification: A solution of 46 $(19 \mathrm{mg}, 0.072 \mathrm{mmol})$ in $\mathrm{MeOH}(4.5 \mathrm{~mL})$ containing $\mathrm{H}_{5} \mathrm{IO}_{6}(33 \mathrm{mg}$, $0.145 \mathrm{mmol}$ ) was stirred at room temperature for $6 \mathrm{~h}$. The mixture was concentrated under reduced pressure and the residue esterified with a solution of diazomethane in $\mathrm{Et}_{2} \mathrm{O}$ at $0 \mathrm{8C}$. The solvent was eliminated and the residue purified by silica gel column chromatography (hexanes/ EtOAc $80: 20)$ to give $80(14 \mathrm{mg}, 0.048 \mathrm{mmol}, 67 \%)$ as a colorless oil: $[\mathrm{a}]_{\mathrm{D}}=+35$ $\mathrm{cm}^{3} \mathrm{~g}^{-1} \mathrm{dm}^{-1}\left(\mathrm{c}=0.87\right.$ in $\left.\mathrm{CHCl}_{3}\right) ;{ }^{1} \mathrm{H} \mathrm{N} \mathrm{MR}\left(500 \mathrm{MHz}, \mathrm{CDCl}_{3}\right): \mathrm{d}=1.98$ (s, $3 \mathrm{H}$ ), 1.71 (ddd, $J=14.5,3.8,3.8 \mathrm{~Hz}, 1 \mathrm{H}$ ), 2.40 (ddd, $J=14.2,8.2,6.0 \mathrm{~Hz}, 1$ H), 3.04 (d, $J=7.3 \mathrm{~Hz}, 1 \mathrm{H}$ ), 3.29 (s, $3 \mathrm{H}$ ), 3.33 (s, $3 \mathrm{H}$ ), 3.37 (s, $3 \mathrm{H}), 3.69$ (s, $3 \mathrm{H}$ ), 3.84 (d, $J=10.1 \mathrm{~Hz}, 1 \mathrm{H}$ ), 3.91 (dd, $J=6.0,3.5 \mathrm{~Hz}, 1 \mathrm{H}$ ), 4.19 (d, $J=$ $10.1 \mathrm{~Hz}, 1 \mathrm{H}$ ) , $4.30 \mathrm{ppm}$ (ddd, $J=8.1,8.1,4.4 \mathrm{~Hz}, 1 \mathrm{H}) ;{ }^{13} \mathrm{C} \mathrm{N} \mathrm{MR} \mathrm{(125.7}$ $\left.\mathrm{MHz}, \mathrm{CDCl}_{3}\right): \mathrm{d}=21.8\left(\mathrm{CH}_{3}\right), 34.6\left(\mathrm{CH}_{2}\right), 52.0\left(\mathrm{CH}_{3}\right), 54.8(\mathrm{CH}), 57.4$ $\left(\mathrm{CH}_{3}\right), 57.9\left(\mathrm{CH}_{3}\right), 59.1\left(\mathrm{CH}_{3}\right), 69.6\left(\mathrm{CH}_{2}\right), 81.2(\mathrm{CH}), 84.0(\mathrm{CH}), 91.0(\mathrm{C})$, 169.7 (C), $171.4 \mathrm{ppm}(\mathrm{C})$; IR (film): $\tilde{\mathrm{n}^{2}}=2935,1737 \mathrm{~cm}^{-1}$; MS $\left(\mathrm{ESI}^{+}\right): \mathrm{m} / \mathrm{z}$ (\%): 313 (100) $\left[M^{+}+\mathrm{Na}\right]$; HRMS $\left(\mathrm{ESI}^{+}\right)$: $\mathrm{m} / \mathrm{z}$ calcd for $\mathrm{C}_{13} \mathrm{H}_{22} \mathrm{NaO}_{7}$ : $313.1263\left[M^{+}+\mathrm{Na}\right]$; found: 313.1261 ; elemen- tal analysis calcd $(\%)$ for $\mathrm{C}_{13} \mathrm{H}_{22} \mathrm{O}_{7}$ (290.31): C 53.78, H 7.64; found: $\mathrm{C} \quad$ 53.61, H 7.72.

Method B and subsequent diazomethane esterification: A solution of 46 (17 $\mathrm{mg}, 0.065 \mathrm{mmol})$ in dry $\mathrm{CH}_{2} \mathrm{Cl}_{2}(1.3 \mathrm{~mL})$, containing (diacetoxyiodo)benzene ( $25 \mathrm{mg}, 0.078 \mathrm{mmol}$ ) and iodine $(17 \mathrm{mg}, 0.065 \mathrm{mmol})$ was irradiated with a $80 \mathrm{~W}$ tungsten-filament lamp at room temperature under nitrogen for $2.5 \mathrm{~h}$. The mixture was then poured into water and extracted with $\mathrm{CH}_{2} \mathrm{Cl}_{2}$. The organic layer was washed with aqueous sodium thiosul- fate, dried, and concentrated under reduced pressure. The residue was esterified with a solution of diazomethane in $\mathrm{Et}_{2} \mathrm{O}$ at 0 8C. The solvent was eliminated and the residue purified by silica gel column chromatography (hexanes/EtOAc 8:2) to give 80 (13 mg, $0.045 \mathrm{mmol}, 69 \%$ ). Esterification of the pure acid $79(15.2 \mathrm{mg}, 0.058 \mathrm{mmol})$ with an excess of diazomethane in $\mathrm{Et}_{2} \mathrm{O}$ at $08 \mathrm{C}$ afforded $80(9.7 \mathrm{mg}, 0.033 \mathrm{mmol}, 58 \%)$.

Methyl (1S,2R,3S,5R)-2-acetyloxy-3-[(tert-butyldiphenylsilyl)oxy]-2- [(tertbutyl diphenylsilyl)oxy]methyl-5-methoxycyclopentane-1-carboxy- late (82): Method B: A solution of $47(15.9 \mathrm{mg}, 0.022 \mathrm{mmol})$ in dry

$\mathrm{CH}_{2} \mathrm{Cl}_{2} \quad(0.5 \mathrm{~mL})$, containing (diacetoxyiodo)benzene $\quad(8.7 \mathrm{mg}$, $0.027 \mathrm{mmol}$ ) and iodine $(5.7 \mathrm{mg}, 0.022 \mathrm{mmol})$ was irradiated with a $80 \mathrm{~W}$ tungsten-filament lamp at room temperature under nitrogen for $2 \mathrm{~h}$. Saturated aqueous sodium thiosulfate was then added until complete decol- 
orization of the iodine and the solvent was then evaporated at reduced pressure. The residue was purified by silica gel column chromatography (hexanes/EtOAc 1:1) to give acid $81(13.0 \mathrm{mg}, 0.019 \mathrm{mmol}, 82 \%$ ) as a colorless oil. [a $]_{\mathrm{D}}=-9 \mathrm{~cm}^{3} \mathrm{~g}^{-1} \mathrm{dm}^{-1}\left(c=0.75\right.$ in $\left.\mathrm{CHCl}_{3}\right) ;{ }^{1} \mathrm{H} \mathrm{N} \mathrm{MR}$ $\left(500 \mathrm{M} \mathrm{Hz}, \mathrm{CDCl}_{3}\right): \mathrm{d}=0.75(\mathrm{~s}, 9 \mathrm{H}), 1.07$ (s, 9H), 1.47 (ddd, $J=12.9,8.5$, $8.5 \mathrm{~Hz}, 1 \mathrm{H}$ ), 1.83 (s, 3H), 2.09 (ddd, $J=13.3,6.9,6.9 \mathrm{~Hz}, 1 \mathrm{H}), 3.23$ (d, $J=7.9 \mathrm{~Hz}, 1 \mathrm{H}), 3.31(\mathrm{~s}, 3 \mathrm{H}), 3.92(\mathrm{~d}, J=11.7 \mathrm{~Hz}, 1 \mathrm{H}), 4.29$ (ddd, $J=8.2$, $8.2,6.9 \mathrm{~Hz}, 1 \mathrm{H}), 4.33(\mathrm{~d}, J=11.4 \mathrm{~Hz}, 1 \mathrm{H}), 4.65(\mathrm{dd}, J=8.2,6.6 \mathrm{~Hz}, 1 \mathrm{H})$, 7.19-7.64 ppm (m, 20H); ${ }^{13} \mathrm{C} \mathrm{NMR}\left(125.7 \mathrm{MHz}, \mathrm{CDCl}_{3}\right): \mathrm{d}=18.9(\mathrm{C})$ $19.2(\mathrm{C}), 21.4\left(\mathrm{CH}_{3}\right), 26.7\left(3 \times \mathrm{CH}_{3}\right), 26.8\left(3 \times \mathrm{CH}_{3}\right), 38.4\left(\mathrm{CH}_{2}\right), 57.8$ $\left(\mathrm{CH}_{3}\right), 58.8(\mathrm{CH}), 69.2\left(\mathrm{CH}_{2}\right), 75.5(\mathrm{CH}), 78.9(\mathrm{CH}), 89.00(\mathrm{C}), 127.6$ $135.8(20 \times \mathrm{CH}), 131.05$ (C), 131.2 (C), 132.5 (C), 133.1 (C), 169.0 (C) $169.7 \mathrm{ppm}(\mathrm{C}) ; \operatorname{IR}$ (film): $\tilde{\mathrm{n}}=2936,1770,1742 \mathrm{~cm}^{-1}$; MS $\left(\mathrm{ESI}^{+}\right): \mathrm{m} / \mathrm{z}$ (\%): $747(100)\left[M^{+}+\mathrm{Na}\right]$; HRMS $\left(\mathrm{ESI}^{+}\right.$): $\mathrm{m} / \mathrm{z}$ calcd for $\mathrm{C}_{42} \mathrm{H}_{52} \mathrm{NaO}_{7} \mathrm{Si}_{2}$ : 747.3149[ $\left.\mathrm{M}^{+}+\mathrm{Na}\right]$; found: 747.3171; elemental analysis calcd (\%) for $\mathrm{C}_{42} \mathrm{H}_{52} \mathrm{O}_{7} \mathrm{Si}_{2}$ (725.03):C 69.58, H 7.23; found: C 69.58, H 7.24.

Method B and subsequent diazomethane esterification : A solution of 47 $(19.0 \mathrm{mg}, 0.027 \mathrm{mmol})$ in dry $\mathrm{CH}_{2} \mathrm{Cl}_{2}(0.5 \mathrm{~mL})$, containing (diacetoxyiodo)benzene (10.4 mg, $0.032 \mathrm{mmol})$ and iodine $(6.8 \mathrm{mg}, 0.027 \mathrm{mmol})$ was irradiated with a $80 \mathrm{~W}$ tungsten-filament lamp at room temperature under nitrogen for $21 \mathrm{~h}$. Saturated aqueous sodium thiosulfate was then added until complete decolorization of the iodine and the solvent was then evaporated at reduced pressure. The residue was esterified with a solution of diazomethane in $\mathrm{Et}_{2} \mathrm{O}$ at 0 8C. The solvent was eliminated and the residue purified by silica gel column chromatography (hexanes/ EtOAc 9:1) to give $82(11.9 \mathrm{mg}, 0.016 \mathrm{mmol}, 60 \%)$ as a colorless oil: $[\mathrm{a}]_{\mathrm{D}}=+7.6$ $\mathrm{cm}^{3} \mathrm{~g}^{-1} \mathrm{dm}^{-1}\left(c=1.51\right.$ in $\left.\mathrm{CHCl}_{3}\right) ;{ }^{1} \mathrm{H} \mathrm{NMR}\left(500 \mathrm{MHz}, \mathrm{CDCl}_{3}\right): \mathrm{d}=0.92(\mathrm{~s}$ 9H), 1.10 (s, 9H), 1.63 (ddd, $J=12.9,8.2,7.9 \mathrm{~Hz}, 1 \mathrm{H}$ ), 1.81 (s,

$3 \mathrm{H}$ ), 2.09 (ddd, $J=13.0,7.2,7.2 \mathrm{~Hz}, 1 \mathrm{H}$ ), 3.26 (s, $3 \mathrm{H}), 3.48(\mathrm{~d}, J=7.3 \mathrm{~Hz}$, $1 \mathrm{H}$ ), 3.56 (s, $3 \mathrm{H}$ ), 4.10 (ddd, $J=7.6,7.6,7.6 \mathrm{~Hz}, 1 \mathrm{H}$ ), 4.18 (d, $J=10.8 \mathrm{~Hz}$, $1 \mathrm{H}$ ), 4.27 (d, $J=10.7 \mathrm{~Hz}, 1 \mathrm{H}$ ), 4.71 (dd, $J=7.9,6.9 \mathrm{~Hz}, 1 \mathrm{H}$ ), $7.26-7.44$ $(\mathrm{m}, \quad 12 \mathrm{H}), \quad 7.54-7.58(\mathrm{~m}, 4 \mathrm{H}), \quad 7.70-7.71 \mathrm{ppm} \quad(\mathrm{m}, 4 \mathrm{H}) ;{ }^{13} \mathrm{C} \mathrm{NMR}$ $\left(100.6 \mathrm{MHz}, \mathrm{CDCl}_{3}\right): \mathrm{d}=19.1(\mathrm{C}), 19.4(\mathrm{C}), 21.6\left(\mathrm{CH}_{3}\right), 26.9\left(6 \times \mathrm{CH}_{3}\right)$ $37.8\left(\mathrm{CH}_{2}\right), 51.6\left(\mathrm{CH}_{3}\right), 54.0(\mathrm{CH}), 57.2\left(\mathrm{CH}_{3}\right), 65.0\left(\mathrm{CH}_{2}\right), 75.7(\mathrm{CH})$, $80.0(\mathrm{CH}), 90.2(\mathrm{C}), 127.5(2 \times \mathrm{CH}), 127.6(6 \times \mathrm{CH}), 129.58(\mathrm{CH}), 129.62$ $(\mathrm{CH}), 129.68(\mathrm{CH}), 129.72(\mathrm{CH}), 133.0(\mathrm{C}), 131.1(\mathrm{C}), 133.5(\mathrm{C}), 133.8$ (C), $135.8(2 \times \mathrm{CH}), 135.90(2 \times \mathrm{CH}), 135.92(2 \times \mathrm{CH}), 135.94(2 \times \mathrm{CH})$,

$169.9(\mathrm{C}), 171.5 \mathrm{ppm}(\mathrm{C}) ;$ IR (film): $\tilde{n}=2933,1737 \mathrm{~cm}^{-1}$; MS (EI): $\mathrm{m} / \mathrm{z}$ (\%): 681(19) [ $\left.M^{+}-t \mathrm{Bu}\right], 135(100) ; \mathrm{HRMS}\left(\mathrm{ESI}^{+}\right.$): $\mathrm{m} / \mathrm{zcalcd}$ for

$\mathrm{C}_{43} \mathrm{H}_{54} \mathrm{NaO}_{7} \mathrm{Si}_{2}$ : $761.3306\left[M^{+}+\mathrm{Na}\right]$; found: 761.3325 ; elemental analysis calcd (\%) for $\mathrm{C}_{43} \mathrm{H}_{54} \mathrm{O}_{7} \mathrm{Si}_{2}$ (739.06): C 69.88, $\mathrm{H}$ 7.36; found: C 70.07, $\mathrm{H}$ 7.42

Method A and subsequent diazomethane esterification: A solution of 47 (40.7 mg, $0.057 \mathrm{mmol})$ in $\mathrm{MeOH}(3.4 \mathrm{~mL})$ containing $\mathrm{H}_{5} \mathrm{IO}_{6}(26.2 \mathrm{mg}$, $0.115 \mathrm{mmol}$ ) was stirred at room temperature for $20 \mathrm{~h}$. The mixture was concentrated under reduced pressure and the residue esterified with a solution of diazomethane in $\mathrm{Et}_{2} \mathrm{O}$ at $0 \mathrm{8C}$. The solvent was eliminated and the residue purified by silica gel column chromatography (hexanes/ EtOAc 9:1) to give $81(10.9 \mathrm{mg}, 0.015 \mathrm{mmol}, 26 \%$ ). Esterification of pure acid 81 $(17.3 \mathrm{mg}, 0.024 \mathrm{mmol})$ with an excess of diazomethane in $\mathrm{Et}_{2} \mathrm{O}$ at $0 \mathrm{8C}$ afforded 82 (10.7 mg, $0.014 \mathrm{mmol}, 60 \%$ ).

(1S,2R,3S,4R,5S)-2-Acetyloxy-3,4,5-tris(benzyloxy)-2-(benzyloxymethyl)cyclopentanecarboxylic acid (83): A deoxygenated solution of $18(80 \mathrm{mg}$, $0.135 \mathrm{mmol})$ in purified $\mathrm{CDCl}_{3}(2 \mathrm{~mL})$, was placed in a resonance tube and irradiated with a daylight lamp at $30 \mathrm{8C}$ for $4 \mathrm{~h}$. The mixture was concentrated under reduced pressure. The crude residue in EtOH (4 mL) containing $\mathrm{H}_{5} \mathrm{IO}_{6}(61 \mathrm{mg}, 0.269 \mathrm{mmol}$ ) was stirred at room temperature for $3 \mathrm{~h}$. The mixture was concentrated under reduced pressure and the residue purified by silica gel column chromatography (hexanes/EtOAc

$9: 1 ! 1: 1)$ to give $83(48 \mathrm{mg}, 0.079 \mathrm{mmol}, 58 \%$ from 18$)$ as an amorphous solid: $[\mathrm{a}]_{\mathrm{D}}=+11 \mathrm{~cm}^{3} \mathrm{~g}^{-1} \mathrm{dm}^{-1}\left(\mathrm{c}=1.87 \mathrm{in} \mathrm{CHCl}_{3}\right) ;{ }^{1} \mathrm{H} \mathrm{NMR}(500 \mathrm{MHz}$ $\left.\mathrm{CDCl}_{3}\right): \mathrm{d}=1.98(\mathrm{~s}, 3 \mathrm{H}), 3.26(\mathrm{~d}, J=9.1 \mathrm{~Hz}, 1 \mathrm{H}), 3.91(\mathrm{dd}, J=6.6,6.6 \mathrm{~Hz}$, $1 \mathrm{H}), 3.92(\mathrm{~d}, J=10.7 \mathrm{~Hz}, 1 \mathrm{H}), 4.33(\mathrm{~d}, J=10.7 \mathrm{~Hz}, 1 \mathrm{H}), 4.35(\mathrm{~d}, J=$ $6.3 \mathrm{~Hz}, 1 \mathrm{H}), 4.61(\mathrm{~d}, J=11.7 \mathrm{~Hz}, 2 \mathrm{H}), 4.63(\mathrm{dd}, J=9.1,6.9 \mathrm{~Hz}, 1 \mathrm{H}), 4.66$ (d, $J=10.7 \mathrm{~Hz}, 2 \mathrm{H}), 4.67(\mathrm{~d}, J=11.4 \mathrm{~Hz}, 1 \mathrm{H}), 4.68(\mathrm{~d}, J=11.9 \mathrm{~Hz}, 1 \mathrm{H})$, $4.73(\mathrm{~d}, J=11.0 \mathrm{~Hz}, 1 \mathrm{H}), 4.87(\mathrm{~d}, J=11.0 \mathrm{~Hz}, 1 \mathrm{H}), 7.27-7.37 \mathrm{ppm}(\mathrm{m}$, $20 \mathrm{H}) ;{ }^{13} \mathrm{C} \mathrm{NMR}\left(100.6 \mathrm{MHz}, \mathrm{CDCl}_{3}\right): \mathrm{d}=21.6\left(\mathrm{CH}_{3}\right), 56.0(\mathrm{CH}), 71.6$ $\left(\mathrm{CH}_{2}\right), 72.7\left(\mathrm{CH}_{2}\right), 73.6\left(\mathrm{CH}_{2}\right), 73.7\left(\mathrm{CH}_{2}\right), 74.3\left(\mathrm{CH}_{2}\right), 82.1(\mathrm{CH}), 85.2$
$(\mathrm{CH}), 85.4(\mathrm{C}), 86.9(\mathrm{CH}), 127.67(3 \times \mathrm{CH}), 127.71(\mathrm{CH}), 127.9(3 \times \mathrm{CH})$, $128.1(2 \times \mathrm{CH}), 128.2(2 \times \mathrm{CH}), 128.3(2 \times \mathrm{CH}), 128.37(2 \times \mathrm{CH}), 128.39$ $(2 \times \mathrm{CH}), 128.6(\mathrm{CH}), 128.8(2 \times \mathrm{CH}), 135.7(\mathrm{C}), 137.7(\mathrm{C}), 138.0(\mathrm{C})$, 138.3 (C), 169.3 (C), $169.9 \mathrm{ppm}(\mathrm{C})$; IR (film): $\mathrm{n}^{\sim}=2928,1742,1731 \mathrm{~cm}^{-1}$; MS (ESI $\left.{ }^{+}\right): m / z(\%): 633$ (100) $\left[M^{+}+\mathrm{Na}\right]$; HRMS $\left(\mathrm{ESI}^{+}\right): \mathrm{m} / \mathrm{z}$ calcd for $\mathrm{C}_{37} \mathrm{H}_{38} \mathrm{NaO}_{8}$ : 633.2464 [ $M^{+}+\mathrm{Na}$ ]; found: 633.2463; elemental analysis calcd (\%) for $\mathrm{C}_{37} \mathrm{H}_{38} \mathrm{O}_{8}$ (610.69): $\mathrm{C} 72.77, \mathrm{H}$ 6.27; found: $\mathrm{C} 72.68, \mathrm{H} 6.27$. (4aR,5S,6S,7R,7aS)-4a-(Acetyloxy)-6,7-dimethoxy-2,2-dimethylhexahydrocyclopenta $[d][1,3]$ dioxine-5-carboxylic acid (84): A deoxygenated solution of diketone $22(20.0 \mathrm{mg}, 0.066 \mathrm{mmol})$ in dry $\mathrm{C}_{6} \mathrm{D}_{6}(0.3 \mathrm{~mL})$, was placed in a resonance tube and irradiated with a daylight lamp at 36- $408 \mathrm{C}$ for 2.5 h. The mixture was concentrated under reduced pressure and the residue dissolved in $\mathrm{CH} \quad \mathrm{Cl}_{2}(1.3 \mathrm{~mL}$ ) and treated with (diacetoxyiodo)benzene ( $24.6 \mathrm{mg}, 0.076 \mathrm{mmol}$ ) and iodine ( $16.1 \mathrm{mg}, 0.06 \mathrm{mmol})$. The mixture was irradiated with an $80 \mathrm{~W}$ tungsten-filament lamp at room temperature under nitrogen for 40 min. An excess of solid $\mathrm{Na}_{2} \mathrm{~S}_{2} \mathrm{O}_{3}$ was then added and the solvent concentrated under reduced pressure. The residue was purified by silica gel column chromatography (hexanes/ EtOAc $8: 2$ ) to give 84 (17.4 mg, $0.054 \mathrm{mmol}, 82 \%$, from 22 ) as an amorphous solid. [a $]_{\mathrm{D}}=-19 \mathrm{~cm}^{3} \mathrm{~g}^{-1} \mathrm{dm}^{-1}\left(c=0.44\right.$ in $\left.\mathrm{CHCl}_{3}\right) ;{ }^{1} \mathrm{H} \mathrm{N} \mathrm{M} \mathrm{R}$ $\left(400 \mathrm{MHz}, \mathrm{CDCl}_{3}\right): \mathrm{d}=1.41(\mathrm{~s}, 3 \mathrm{H}), 1.60(\mathrm{~s}, 3 \mathrm{H}), 2.00(\mathrm{~s}, 3 \mathrm{H}), 3.39(\mathrm{~s}$, $3 \mathrm{H}), 3.46(\mathrm{~s}, 3 \mathrm{H}), 3.48(\mathrm{~d}, J=3.2 \mathrm{~Hz}, 1 \mathrm{H}), 3.56(\mathrm{~d}, J=9.3 \mathrm{~Hz}, 1 \mathrm{H}), 4.21$ (d, $J=11.9 \mathrm{~Hz}, 1 \mathrm{H}$ ), 4.29 (ddd, $J=9.3,3.2,1.1 \mathrm{~Hz}, 1 \mathrm{H}$ ), 4.53 (d, $J=$ $11.9 \mathrm{~Hz}, 1 \mathrm{H}), 4.79 \mathrm{ppm}(\mathrm{d}, J=1.0 \mathrm{~Hz}, 1 \mathrm{H}) ;{ }^{13} \mathrm{C}$ NMR $(100.6 \mathrm{MHz}$, $\left.\mathrm{CDCl}_{3}\right): \mathrm{d}=19.8\left(\mathrm{CH}_{3}\right), 21.6\left(\mathrm{CH}_{3}\right), 28.2\left(\mathrm{CH}_{3}\right), 52.1(\mathrm{CH}), \quad 57.7\left(\mathrm{CH}_{3}\right)$ $58.2\left(\mathrm{CH}_{3}\right), 59.5\left(\mathrm{CH}_{2}\right), 73.8(\mathrm{CH}), 83.3(\mathrm{C}), 86.8(\mathrm{CH}), 89.9(\mathrm{CH}), 99.1$ (C), 170.2 (C), $173.2 \mathrm{ppm}(\mathrm{C})$; IR $\left(\mathrm{CHCl}_{3}, 0.2 \mathrm{~mm}\right): \tilde{n}=3504,3016,1741$, $1717 \mathrm{~cm}^{-1}$; MS (ESI $\left.{ }^{+}\right): m / z(\%): 341(100)\left[M^{+}+\mathrm{Na}\right]$; HRMS $\left(\mathrm{ESI}^{+}\right)$: $m / z$ calcd for $\mathrm{C}_{14} \mathrm{H}_{22} \mathrm{NaO}_{8}: 341.1212$ [ $M^{+}+\mathrm{Na}$ ]; found: 341.1207; elemen- tal analysis calcd (\%) for $\mathrm{C}_{14} \mathrm{H}_{22} \mathrm{O}_{8}$ (318.32): C 52.82, $\mathrm{H}$ 6.97; found: $\mathrm{C} 52.86$, H 6.92.

(1S,2R,5R)-2-(Acetyloxy)-5-methoxy-2-[(tert-butyldiphenylsilyl)oxy]methyl-1-cyclopentanecarboxylic acid (85) from photocyclization-fragmentation of 42: A deoxygenated solution of $42(40.8 \mathrm{mg}, 0.090 \mathrm{mmol})$ in $\operatorname{dry~}_{6} \mathrm{D}_{6}(0.3 \mathrm{~mL})$, was placed in a resonance tube and irradiated with a daylight lamp at $36-408 \mathrm{C}$ for $5 \mathrm{~h}$. The solution washeated to $608 \mathrm{C}$ for $2 \mathrm{~h}$ in the dark and concentrated under reduced pressure. The crude residue in $\mathrm{MeOH}(3.0 \mathrm{~mL})$, containing $\mathrm{H} \mathrm{I}_{6}(45.4 \mathrm{mg}, 0.2 \mathrm{mmol})$, was stirred at room temperature for $3 \mathrm{~h}$. The mixture was concentrated under reduced pressure and the residue purified by silica gel column chromatography (hexanes/EtOAc $8: 2$ !EtO Ac) to give $85(10.5 \mathrm{mg}, 0.022 \mathrm{mmol}$, $46 \%)$ as an amorphous solid. [a] $]_{\mathrm{D}}=+1 \mathrm{~cm}^{3} \mathrm{~g}^{-1} \mathrm{dm}^{-1}\left(c=0.52\right.$ in $\left.\mathrm{CHCl}_{3}\right)$; ${ }^{1} \mathrm{H} \mathrm{NMR}\left(400 \mathrm{MHz}, \mathrm{CDCl}_{3}\right): \mathrm{d}=1.10(\mathrm{~s}, 9 \mathrm{H}), 1.59(\mathrm{~m}, 1 \mathrm{H}), 1.98(\mathrm{~s}, 3 \mathrm{H})$, 2.03-2.12 (m, 2 H), $2.21(\mathrm{~m}, 1 \mathrm{H}), 3.18(\mathrm{~d}, J=6.3 \mathrm{~Hz}, 1 \mathrm{H}), 3.38(\mathrm{~s}, 3 \mathrm{H})$, $3.78(\mathrm{~d}, J=10.4 \mathrm{~Hz}, 1 \mathrm{H}), 4.386(\mathrm{~d}, J=10.4 \mathrm{~Hz}, 1 \mathrm{H}$ ), 4.387 (ddd, $J=6.3$, $6.3,6.3 \mathrm{~Hz}, 1 \mathrm{H}), 7.38-7.48(\mathrm{~m}, 6 \mathrm{H}), 7.63-7.67 \mathrm{ppm}(\mathrm{m}, 4 \mathrm{H}) ;{ }^{13} \mathrm{C} \mathrm{NMR}$ $\left(125.7 \mathrm{MHz}, \mathrm{CDCl}_{3}\right): \mathrm{d}=19.2(\mathrm{C}), 21.8\left(\mathrm{CH}_{3}\right), 26.8\left(3 \times \mathrm{CH}_{3}\right), 29.2$ $\left(\mathrm{CH}_{2}\right), 32.1\left(\mathrm{CH}_{2}\right), 57.4\left(\mathrm{CH}_{3}\right), 59.0(\mathrm{CH}), 67.2\left(\mathrm{CH}_{2}\right), 82.5(\mathrm{CH}), 88.9$ (C), $127.9(2 \times \mathrm{CH}), 128.0(2 \times \mathrm{CH}), 130.3(2 \times \mathrm{CH}), 131.6(\mathrm{C}), 131.8(\mathrm{C})$, $135.6(2 \times \mathrm{CH}), 135.7(2 \times \mathrm{CH}), 169.7(\mathrm{C}), 170.8 \mathrm{ppm}(\mathrm{C})$; IR $\left(\mathrm{CHCl}_{3}\right.$, $0.2 \mathrm{~mm}): \tilde{n}=2938,1739 \mathrm{~cm}^{-1}$; MS $\left(\mathrm{ESI}^{+}\right): \mathrm{m} / \mathrm{z}(\%): 493(100)\left[\mathrm{M}^{+}+\mathrm{Na}\right]$; HRMS ( $\mathrm{ESI}^{+}$): $m / z$ calcd for $\mathrm{C}_{26} \mathrm{H}_{34} \mathrm{NaO}_{6} \mathrm{Si}: 493.2022\left[M^{+}+\mathrm{Na}\right]$; found: 493.2021; elemental analysis calcd (\%) for $\mathrm{C}_{26} \mathrm{H}_{34} \mathrm{O}_{6} \mathrm{Si}$ (470.63): $\mathrm{C} 66.35, \mathrm{H}$ 7.28; found: C 66.12, H 7.03.

Methyl (1S,2R,3S,4R,5S)-3,4,5-tris(benzyloxy)-2-(benzyloxy)methyl]cyclopentanecarboxylate (86): A solution of $83(19 \mathrm{mg}, 0.031 \mathrm{mmol})$ in methanolic $\mathrm{KOH}(0.6 \mathrm{~mL}, 4 \mathrm{mg}, 0.078 \mathrm{mmol})$ was stirred at room temperature for $24 \mathrm{~h}$. The mixture was neutralized with acid resin Dowex $50 \mathrm{~W} \times 8$ $\left(\mathrm{H}^{+}\right)$, filtered and concentrated under reduced pressure. A solu- tion of the residue in $\mathrm{Et}_{2} \mathrm{O}$ was treated with an excess of diazomethane at $08 \mathrm{C}$ and concentrated under reduced pressure. The residue was puri- fied by silica gel column chromatography (hexanes/EtOAc 8:2) to give 86 (18 mg, 0.031 mmol, $99 \%$ ): colorless oil. [a] $]_{\mathrm{D}}=+2 \mathrm{~cm}^{3} \mathrm{~g}^{-1} \mathrm{dm}^{-1}\left(c=0.25\right.$ in $\left.\mathrm{CHCl}_{3}\right) ;{ }^{1} \mathrm{H}$ NMR $\left(500 \mathrm{MHz} \mathrm{CDCl}_{3}\right): \mathrm{d}=3.11(\mathrm{~d}, J=8.0 \mathrm{~Hz}, 1 \mathrm{H}), 3.64(\mathrm{~s}, 3 \mathrm{H}), 3.69$ (d, $J=9.5 \mathrm{~Hz}, 1 \mathrm{H}), 3.75(\mathrm{~d}, J=9.5 \mathrm{~Hz}, 1 \mathrm{H}), 3.89$ (d, $J=$ $3.7 \mathrm{~Hz}, 1 \mathrm{H}), 3.94(\mathrm{dd}, J=4.8,4.0 \mathrm{~Hz}, 1 \mathrm{H}), 4.54(\mathrm{~m}, 1 \mathrm{H}), 4.52-4.62(\mathrm{~m}$, $8 \mathrm{H}), 7.25-7.35 \mathrm{ppm}(\mathrm{m}, 20 \mathrm{H}) ;{ }^{13} \mathrm{C} \mathrm{NMR}\left(125.7 \mathrm{MHz} \mathrm{CDCl}_{3}\right): \mathrm{d}=51.9$ $\left(\mathrm{CH}_{3}\right), 54.0(\mathrm{CH}), 72.0\left(\mathrm{CH}_{2}\right), 72.30\left(\mathrm{CH}_{2}\right), 72.34\left(\mathrm{CH}_{2}\right), 72.8\left(\mathrm{CH}_{2}\right), 73.7$ 
$\left(\mathrm{CH}_{2}\right), 81.0(\mathrm{C}), 85.1(\mathrm{CH}), 86.6(\mathrm{CH}), 87.2(\mathrm{CH}), 127.6-128.3(20 \times \mathrm{CH})$, 137.9 (C), 138.05 (C), $138.09(2 \times \mathrm{C}), 172.8 \mathrm{ppm}$ (C); IR (film): $\mathrm{n}^{\sim}=3499$, 1732, $1068 \mathrm{~cm}^{-1}$; MS $\left(\mathrm{ESI}^{+}\right): \mathrm{m} / \mathrm{z}(\%): 605(100)\left[M^{+}+\mathrm{Na}\right]$; HRMS $\left(\mathrm{ESI}^{+}\right.$): $\mathrm{m} / \mathrm{z}$ calcd for $\mathrm{C}_{36} \mathrm{H}_{38} \mathrm{NaO}_{7}: 605.2515\left[\mathrm{M}^{+}+\mathrm{Na}\right.$ ]; found: 605.2513 elemental analysis calcd (\%) for $\mathrm{C}_{36} \mathrm{H}_{38} \mathrm{O}_{7}$ (582.68): $\mathrm{C}$ 74.21, H 6.57; found: C 74.42 , H 6.60

(1S,2S,3R,4S,5R)-2,3,4-Tris(benzyloxy)-5-(benzyloxy)methyl-6-oxabicyclo[3.2.0]heptan-7-one (87): A solution of $83(15 \mathrm{mg}, 0.025 \mathrm{mmol}$ ) in methanolic $\mathrm{K} \mathrm{OH}(0.5 \mathrm{~mL}, 4 \mathrm{mg}, 0.071 \mathrm{mmol})$ was stirred at room temperature for $24 \mathrm{~h}$, neutralized with acid ion-exchange resin Dowex-50 W $\times 8$ $\left(\mathrm{H}^{+}\right)$, filtered and concentrated under reduced pressure. Benzenesulfonyl chloride (16 $\mathrm{mL}, 0.130 \mathrm{mmol}$ ) was added to a solution of the residue in dry pyridine $(0.1 \mathrm{~mL})$ and the mixture stirred at $08 \mathrm{C}$ for $14 \mathrm{~h}$. The solu- tion was then poured into ice-water and extracted with $\mathrm{Et}_{2} \mathrm{O}$. The residue was purified by silica gel column chromatography (hexanes/EtOAc 8:2) to give $87(10 \mathrm{mg}, 0.018 \mathrm{mmol}, 73 \% \text {, two-step) as a colorless oil. [a }]_{\mathrm{D}}=+2 \mathrm{~cm}^{3} \mathrm{~g}^{-}$ ${ }^{1} \mathrm{dm}^{-1}\left(c=0.50\right.$ in $\left.\mathrm{CHCl}_{3}\right) ;{ }^{1} \mathrm{H} \mathrm{NMR}\left(500 \mathrm{MHz} \mathrm{CDCl}_{3}\right): \mathrm{d}=3.97(\mathrm{~d}, J=$ $12.0 \mathrm{~Hz}, 1 \mathrm{H}$ ), $3.99(\mathrm{~d}, J=11.7 \mathrm{~Hz}, 1 \mathrm{H}), 4.00\left(\mathrm{dd}, J=1.5 \mathrm{~Hz}, J_{\mathrm{W}}=\right.$ $1.4 \mathrm{~Hz}, 1 \mathrm{H}$ ), 4.16 (ddd, $J=2.4,2.0 \mathrm{~Hz}, J_{\mathrm{W}}=1.4 \mathrm{~Hz}, 1 \mathrm{H}$ ), 4.19 (d, $J=$ $4.7 \mathrm{~Hz}, 1 \mathrm{H}$ ) , 4.22 (dd, $J=1.9,1.9 \mathrm{~Hz}, 1 \mathrm{H}$ ), 4.47 (d, $J=12.0 \mathrm{~Hz}, 1 \mathrm{H}$ ), 4.48 (d, $J=12.0 \mathrm{~Hz}, 1 \mathrm{H}), 4.52(\mathrm{~d}, J=11.7 \mathrm{~Hz}, 1 \mathrm{H}), 4.52(\mathrm{~d}, J=12.0 \mathrm{~Hz}, 1 \mathrm{H})$, $4.53(\mathrm{~d}, J=12.3 \mathrm{~Hz}, 1 \mathrm{H}$ ), 4.57 (d, $J=12.0 \mathrm{~Hz}, 1 \mathrm{H}), 4.60$ (d, $J=12.3 \mathrm{~Hz}$, $1 \mathrm{H}), \quad 4.63(\mathrm{~d}, J=12.0 \mathrm{~Hz}, 1 \mathrm{H}), 7.22-7.36 \mathrm{ppm}(\mathrm{m}, 20 \mathrm{H}) ;{ }^{13} \mathrm{CNMR}$ $\left(100.6 \mathrm{MHz}, \mathrm{CDCl}_{3}\right): \mathrm{d}=60.5(\mathrm{CH}), 67.6\left(\mathrm{CH}_{2}\right), 71.1\left(\mathrm{CH}_{2}\right), 72.1\left(\mathrm{CH}_{2}\right)$, $73.0\left(\mathrm{CH}_{2}\right), 73.8\left(\mathrm{CH}_{2}\right), 81.4(\mathrm{CH}), 82.9(\mathrm{CH}), 86.8(\mathrm{CH}), 88.1(\mathrm{C}), 127.6$ $128.5(20 \times \mathrm{CH}), 137.2(\mathrm{C}), 137.29$ (C), $137.34(\mathrm{C}), 137.4(\mathrm{C}), 167.1 \mathrm{ppm}$ (C); IR (film): $\tilde{n}=2918,1828,1087 \mathrm{~cm}^{-1}$; MS $\left(\mathrm{ESI}^{+}\right): \mathrm{m} / \mathrm{z}(\%): 573(100)$ $\left[M^{+}+\mathrm{Na}\right]$; HRMS $\left(\mathrm{ESI}^{+}\right): \mathrm{m} / z$ calcd for $\mathrm{C}_{35} \mathrm{H}_{34} \mathrm{NaO}_{6}: 573.2253\left[\mathrm{M}^{+}\right.$ $+\mathrm{Na}$ ]; found: 573.2249 ; elemental analysis (\%) for $\mathrm{C}_{35} \mathrm{H}_{34} \mathrm{O}_{6}(550.64)$ : C 76.34,H 6.22; found: C 76.29, H 6.47.

(1R,2S,3R,4S,5S)-2,3,4-Tris(benzyloxy)-1-(benzyloxy)methyl-5-(hydroxymethyl)cyclopentanol (88): $\mathrm{LiAlH}_{4}(6 \mathrm{mg}, 0.082 \mathrm{mmol})$ was added to a solution of $83(25 \mathrm{mg}, 0.041 \mathrm{mmol})$ in dry THF $(1.6 \mathrm{~mL})$ and the suspension stirred at $08 \mathrm{C}$ under nitrogen for $4 \mathrm{~h}$. A saturated solution of $\mathrm{Na}_{2} \mathrm{SO}_{4}$ was then added, the white solid mass precipitate was filtered through Celite, and the solvent concentrated under reduced pressure. The residue was purified by silica gel column chromatography (hexanes/

Et OAc $1: 1 ! 4: 6)$ to give $88(18 \mathrm{mg}, 0.032 \mathrm{mmol}, 78 \%)$ as a colorless oil: $[\mathrm{a}]_{\mathrm{D}}=-8.2 \mathrm{~cm}^{3} \mathrm{~g}^{-1} \mathrm{dm}^{-1}\left(c=0.11\right.$ in $\left.\mathrm{CHCl}_{3}\right) ;{ }^{1} \mathrm{H} \mathrm{NMR}\left(500 \mathrm{MHz}, \mathrm{CDCl}_{3}\right)$ : $\mathrm{d}=2.21$ (ddd, $J=9.3,6.0,3.7 \mathrm{~Hz}, 1 \mathrm{H}$ ), $3.61(\mathrm{~d}, J=9.5 \mathrm{~Hz}, 1 \mathrm{H}), 3.76(\mathrm{~d}$, $J=9.1 \mathrm{~Hz}, 1 \mathrm{H}), 3.79$ (dd, $J=11.6,6.0 \mathrm{~Hz}, 1 \mathrm{H}), 3.83(\mathrm{~d}, J=4.7 \mathrm{~Hz}, 1 \mathrm{H})$, 3.87 (dd, $J=11.6,3.5 \mathrm{~Hz}, 1 \mathrm{H}$ ), 3.91 (dd, $J=5.4,5.4 \mathrm{~Hz}, 1 \mathrm{H}$ ), 4.09 (dd, $J=$ $8.8,5.4 \mathrm{~Hz}, 1 \mathrm{H}), 4.45-4.73(\mathrm{~m}, 8 \mathrm{H}), 7.25-7.38 \mathrm{ppm}(\mathrm{m}, 20 \mathrm{H}) ;{ }^{1} \mathrm{H}$ NMR $\left(500 \mathrm{MHz}, \mathrm{C}_{6} \mathrm{D}_{6}\right): \mathrm{d}=2.24(\mathrm{brs}, 1 \mathrm{H}), 2.31$ (ddd, $J=4.7,4.7,9.5 \mathrm{~Hz}, 1 \mathrm{H}$ ) 3.08 (br s, $1 \mathrm{H}$ ), 3.51 (d, $J=9.5 \mathrm{~Hz}, 1 \mathrm{H}), 3.68(\mathrm{~d}, J=9.5 \mathrm{~Hz}, 1 \mathrm{H}), 3.84(\mathrm{br}$ t, $J=4.4 \mathrm{~Hz}, 2 \mathrm{H}), 3.93(\mathrm{~d}, J=5.4 \mathrm{~Hz}, 1 \mathrm{H}), 4.05(\mathrm{dd}, J=5.7,5.7 \mathrm{~Hz}, 1 \mathrm{H}$ ), $4.22(\mathrm{~s}, 2 \mathrm{H}), 4.25(\mathrm{dd}, J=6.0,8.5 \mathrm{~Hz}, 1 \mathrm{H}), 4.54(\mathrm{~d}, J=12.0 \mathrm{~Hz}, 1 \mathrm{H}), 4.57$ $(\mathrm{d}, J=11.0 \mathrm{~Hz}, 1 \mathrm{H}), 4.57(\mathrm{~d}, J=11.0 \mathrm{~Hz}, 1 \mathrm{H}), 4.62(\mathrm{~d}, J=11.7 \mathrm{~Hz}, 1 \mathrm{H})$, $4.64(\mathrm{~d}, J=11.5 \mathrm{~Hz}, 1 \mathrm{H}), 4.67(\mathrm{~d}, J=11.7 \mathrm{~Hz}, 1 \mathrm{H}), 7.05-7.36 \mathrm{ppm}(\mathrm{m}$, $20 \mathrm{H}) ;{ }^{13} \mathrm{C} \mathrm{NMR}(100.6 \mathrm{MHz}, \mathrm{CDCl}): \mathrm{d}=49.9(\mathrm{CH}), 60.5(\mathrm{CH}), 72.2$ $\left(\mathrm{CH}_{2}\right), 72.6\left(\mathrm{CH}_{2}\right), 72.6\left(\mathrm{CH}_{2}\right), 73.1\left(\mathrm{CH}_{2}\right), 73.9\left(\mathrm{CH}_{2}\right), 80.6(\mathrm{C}), 82.7$ $(\mathrm{CH}), 86.9(\mathrm{CH}), 87.8(\mathrm{CH}), 127.7-128.5(20 \times \mathrm{CH}), 137.4(\mathrm{C}), 138.1(\mathrm{C})$, $138.2(\mathrm{C}), 138.4 \mathrm{ppm}(\mathrm{C}) ;{ }^{13} \mathrm{C} \mathrm{NMR}\left(125.7 \mathrm{MHz}, \mathrm{C}_{6} \mathrm{D}_{6}\right): \mathrm{d}=50.0(\mathrm{CH})$, $60.9\left(\mathrm{CH}_{2}\right), 72.2\left(\mathrm{CH}_{2}\right), 72.6\left(\mathrm{CH}_{2}\right), 72.8\left(\mathrm{CH}_{2}\right), 73.6\left(\mathrm{CH}_{2}\right), 73.7\left(\mathrm{CH}_{2}\right)$, $80.7(\mathrm{C}), 82.8(\mathrm{CH}), 87.7(\mathrm{CH}), 88.6(\mathrm{CH}), 127.6-128.6(20 \times \mathrm{CH}), 138.3$ (C), 139.0 (C), 139.2 (C), 139.3 ppm (C); IR (film): $\tilde{n}=3445,2918,1075$ $\mathrm{cm}^{-1}$; MS (ESI): $m / z(\%): 577$ (100) $\left[M^{+}+\mathrm{Na}\right]$; HRM S $\left(\mathrm{ESI}^{+}\right): \mathrm{m} / z$ calcd for $\mathrm{C}_{35} \mathrm{H}_{38} \mathrm{NaO}_{6}$ : $577.2566\left[M^{+}+\mathrm{Na}\right]$; found: 577.2564; elemental analysis calcd (\%) for $\mathrm{C}_{35} \mathrm{H}_{38} \mathrm{O}_{6}$ (554.67): $\mathrm{C} 73.79, \mathrm{H}$ 6.91; found: $\mathrm{C} 73.39, \mathrm{H}$ 7.31 .

Methyl (3R,4S,5S)-3,4,5-tris(benzyloxy)-2-(benzyloxy)methyl-1-cyclopentene-1-carboxylate (89): $\mathrm{Tf}_{2} \mathrm{O}(14 \mathrm{~mL}, 0.081 \mathrm{mmol})$ was added dropwise to a solution of $86(16 \mathrm{mg}, 0.027 \mathrm{mmol})$ in dry $\mathrm{CH}_{2} \mathrm{Cl}_{2}$ containing dry pyri- dine $(14 \mathrm{~mL})$ at $0 \mathrm{8C}$. The mixture was stirred at room temperature for $24 \mathrm{~h}$, and concentrated under reduced pressure. The residue was purified by silica gel column chromatography (hexanes/EtOAc 8:2) to give 89 (14 mg, $0.025 \mathrm{mmol}, 93 \%)$ as a colorless oil. $[\mathrm{a}]_{\mathrm{D}}=-16 \mathrm{~cm}^{3} \mathrm{~g}^{-1} \mathrm{dm}^{-1}(c=$

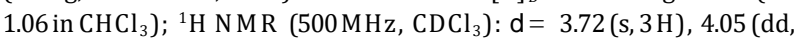

$J=2.6,2.4 \mathrm{~Hz}, 1 \mathrm{H}), 4.37(\mathrm{~d}, J=13.8 \mathrm{~Hz}, 1 \mathrm{H}), 4.45-4.71(\mathrm{~m}, 8 \mathrm{H}), 4.63(\mathrm{~m}$, $1 \mathrm{H}), 4.69(\mathrm{~m}, 1 \mathrm{H}), 4.83(\mathrm{~d}, J=13.8 \mathrm{~Hz}, 1 \mathrm{H}), 7.26-7.36 \mathrm{ppm}(\mathrm{m}, 20 \mathrm{H})$; ${ }^{13} \mathrm{CNMR}\left(100.6 \mathrm{MHz}, \mathrm{CDCl}_{3}\right): \mathrm{d}=51.6\left(\mathrm{CH}_{3}\right), 64.6\left(\mathrm{CH}_{2}\right), 71.8\left(\mathrm{CH}_{2}\right)$ $72.2\left(\mathrm{CH}_{2}\right), 72.6\left(\mathrm{CH}_{2}\right), 72.9\left(\mathrm{CH}_{2}\right), 85.8(\mathrm{CH}), 85.9(\mathrm{CH}), 87.8(\mathrm{CH})$, 127.6-128.4 (20× CH), 131.9 (C), 137.9 (C), 138.0 (C), 138.26 (C), 138.35 (C), $153.2(C), 164.8 \mathrm{ppm}(\mathrm{C})$; IR (film): $\mathrm{n}^{-}=2922,1722,1070 \mathrm{~cm}^{-1}$; MS $\left(\mathrm{ESI}^{+}\right): \mathrm{m} / \mathrm{z}(\%): 587$ (100) $\left[\mathrm{M}^{+}+\mathrm{Na}\right]$; HRMS $\left(\mathrm{ESI}^{+}\right): \mathrm{m} / \mathrm{z}$ calcd for $\mathrm{C}_{36} \mathrm{H}_{36} \mathrm{NaO}_{6}$ : 587.2401[ $\left.M^{+}+\mathrm{Na}\right]$; found: 587.2405; elemental analysis calcd (\%) for $\mathrm{C}_{36} \mathrm{H}_{36} \mathrm{O}_{6}$ (564.67): C 76.57, $\mathrm{H}$ 6.43; found: $\mathrm{C} 76.77, \mathrm{H} \quad 6.63$.

\section{Acknowledgements}

This work was supported by the Investigation Programs (CTQ201018244) of the Ministerio de Economía y Competitividad and (SolSub20081000192) of the Gobierno de Canarias. D.A.-D. thanks the Ministerio de Economía y Competitividad for a fellowship. C.R.-F. thanks the Programa I3P-CSIC for a postdoctoral contract.

[1] For reviews on Norrish/Yang reaction, see: a) P. J. Wagner in Syn- thetic Organic Photochemistry (Molecular and Supramolecular Photochemistry), Vol. 12 (Eds.: A. G. Griesbeck, J. Mattay), Marcel Dekker, New York, 2005, pp. 11 - 39; b) P. Wessig, 0. Mühling in Synthetic Organic Photochemistry (Molecular and Supramolecular Photochemistry), Vol. 12 (Eds.: A. G. Griesbeck, J. Mattay), Marcel Dekker, New York, 2005, pp. 41 - 87; c) P. J. Wagner, P. Klán in Handbook of Organic Photochemistry and Photobiology, Vol. 1 (Eds.: W. M. Horspool, F. Lenci), CRC, Boca Raton, 2003, chap. 52, pp. 1 - 31; d) T. Hasegawa in Handbook of Organic Photochemistry and Photobiology, Vol. 1 (Eds.: W. M. Horspool, F. Lenci), CRC, Boca Raton, 2003, chap. 55, pp. 1 - 23; e) P. Wessig in Handbook of Organic Photochemistry and Photobiology, Vol. 1 (Eds.: W. M. Horspool, F. Lenci), CRC, Boca Raton, 2003, chap. 57, pp. 1 - 20; f) P. J. Wagner in Handbook of Organic Photochemistry and Photobiology, Vol. 1 (Eds.: W. M. Horspool, F. Lenci), CRC, Boca Raton, 2003, chap. 58, pp. 1 - 70; g) P. J. Wagner, B.-S. Park in Organic Photochemistry, Vol. 11 (Ed.: A. Padwa), Marcel Dekker, New York, 1991, pp. 227 -365; h) P. J. Wagner, Acc. Chem. Res. 1989, 22, 83-91.

[2] a) N. C. Yang, D.-D. H. Yang, J.Am. Chem. Soc. 1958, 80,2913- 2914; b) W. H. Urry, D. J. Trecker, J.Am. Chem. Soc. 1962, 84, 118$\underline{120}$

3 a) N. K. Hamer, TetrahedronLett. 1982,23,473-474; b) N. K. Hamer, J.Chem. Soc. Perkin Trans. 1 1983,61-64; c) N. K. Hamer, Tetrahedron Lett. 1986, 27, 2167-2168; d) M. Obayashi, E. Mizuta, S. Noguchi, Chem. Pharm. Bull. 1979,27, 1679- 1682.

4 a) V. B. Kurteva, C. A. M. Alonso, Chem. Rev. 2009, 109, 6809- 6857; b) O. Arjona, A. M. Gómez, J. C. López, J. Plumet, Chem. Rev. 2007, 107, 1919-2036; c) M. Sollogoub, P. Sinaÿ in The Organic Chemistry of Sugars (Eds.: D. E. Levy, P. Fügedi), CRC, Boca Raton, 2006, pp. 349 - 381; d) R. J. Ferrier, S. Middleton, Chem. Rev. 1993, 93, $2779-$ 2831.

5 a) P. I. Dalko, P. Sinaÿ, Angew. Chem. 1999, 111, 819 - 823; Angew. Chem. Int. Ed. 1999, 38,773 - 777; b) H. Redlich, Angew. Chem. 1994, 106, 1407-1409; Angew. Chem. Int. Ed. Engl. 1994,33, 13451347.

6 a) T. Nakahara, N. Okamoto, K. Suzuki, O. Kanie, Carbohydr.Res. 2008, 343,1624-1635; b) L. A. Paquette, R. M. Arbit, J. A. Funel, S. Bolshakov, Synthesis 2002, 2105-2109; c) D. J. Jenkins, A. M. Riley, B. V. L. Potter, J. Org. Chem. 1996, 61, 7719 - 7726; d) Y. Yoshida, T. Nakagawa, F. Sato, Synlett 1996, 437 - 438; e) Y. Hanzawa, H. Ito, T. Taguchi, Symlett 1995, 299- 305; f) H. Ito, Y. Ikeuchi, T. Taguchi, Y. Hanzawa, J. Am. Chem. Soc. 1994, 116, 5469-5470; g) H. Ito, Y. Motoki, T. Taguchi, Y. Hanzawa, J.Am. Chem. Soc. 1993, 115, 8835 8836.

7 J. M. Aurrecoechea, B. Lopez, M. Arrate, J.Org. Chem. 2000,65, 6493 $-6501$. 
8 T. Kan, S. Nara, T. Ozawa, H. Shirahama, F. Matsuda, Angew. Chem. 2000, 112, 363 - 365; Angew. Chem. Int. Ed. 2000,39, 355- 357.

9 a) J. J. C. Gronjé, C. W. Holzapfel, D. B. G. Williams, Tetrahedron Lett. 1996, 37, 5817 - 5820; b) J. L. Chiara, S. Martínez, M. Bernabé, $J$. Org. Chem. 1996, 61, 6488-6489.

10 A. Chénedé, P. Pothier, M. Sollogoub, A. J. Fairbanks, P. Sinaÿ, J. Chem. Soc. Chem. Commun. 1995, 1373- 1374.

11 a) A. J. Herrera, M. Rondón, E. Suárez, Synlett 2007,1851-1856 b) A. J. Herrera, M. Rondón, E. Suárez, J.Org. Chem. 2008, 73, 3384$\underline{3391 .}$

12 a) A. Martín, I. Pérez-Martín, L. M. Quintanal, E. Suárez, Org. Lett. 2007,9,1785-1788; b) C. G. Francisco, A. J. Herrera, A. R. Kennedy, D. Melián, E. Suárez, Angew. Chem. 2002, 114, 884-886; Angew. Chem. Int. Ed. 2002, 41, 856-858; c) C. G. Francisco, A. J. Herrera, A. R. Kennedy, A. Martín, D. Melián, I. Peréz-Martín, L. M. Quintanal, E. Suárez, Chem. Eur. J.2008,14, 10369-10381.

13 Preliminary results of this work have been published: D. ÁlvarezDorta, E. I. León, A. R. Kennedy, C. Riesco-Fagundo, E. Suárez, Angew. Chem. 2008, 120, 9049-9051; Angew. Chem. Int. Ed. 2008, 47, 8917-8919.

14 C. G. Francisco, A. J. Herrera, E. Suárez, J.Org. Chem. 2002,67, 7439 $-7445$.

15 a) C. G. Francisco, R. Freire, A. J. Herrera, I. Pérez-Martín, E. Suárez, Org. Lett. 2002, 4, 1959-1961; b) C. G. Francisco, A. J. Herrera, E. Suárez, Tetrahedron Lett. 2000,41, 7869- 7873.

16 For a feature article, see: a) M. Isobe, R. Nishizawa, S. Hosokawa,

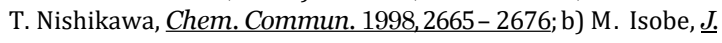
Synth. Org. Chem. Jpn. 1994, 52, 968-979; c) T. Nishikawa, M. Ishikawa, M. Isobe, Synlett 1999,123-125. For a recent indium-mediat- ed alkynylation, see: d) N. Lubin-Germain, J.-P. Baltaze, A. Coste, A. Hallonet, H. Lauréano, G. Legrave, J. Uziel, J. Augé, Org. Lett. 2008,10,725-728.

17 a) A. Dondoni, G. Mariotti, A. Marra, J.Org. Chem. 2002,67, 44754486; b) L. F. Tietze, U. Griesbach, I. Schuberth, U. Bothe, A. Marra, A. Dondoni, Chem. Eur. J.2003, 9, 1296-1302.

18 Precedents for such difficulties can be found in the literature, see: T. M. Davis, T. L. Lowary, Carbohydr. Res. 2000,324, 210- 217.

[19] a) M. W. Logue, K. Teng, J.Org. Chem. 1982, 47, 2549-2553. See also: b) E. T. Bogoradovskii, V. S. Zavgorodnii, A. A. Petrov, $Z h$. Obshch. Khim. 1977, 47, 1548- 1551.

[20] a) J. Xu, A. Egger, B. Bernet, A. Vasella, Helv.Chim. Acta 1996, 79 2004-2012; b) T. Lowary, M. Mendal, A. Helmboldt, A. Vasella, K. Bock, J.Org. Chem. 1998,63, 9657-9668.

[21] a) P. S. Bailey, Chem. Rev. 1958, 58, 925 - 1010; b) T. F. Favino, G. Fronza, C. Fuganti, D. Fuganti, P. Graselli, A. Mele, J.Org.Chem. 1996, 61, 8975-8979; c) L. Re, B. Maurer, G. Ohloff, Helv.Chim. Acta $1973,56,1882-1894$

22 a) M. Schrçder, Chem. Rev. 1980, 80, 187 - 213; b) R. Zibuck, D. Seebach, Helv. Chim. Acta 1988,71, 237-240.

23 CCDC-695280 (2) contains the supplementary crystallographic data for this paper. These data can be obtained free of charge from The Cambridge Crystallographic Data Centre via www.ccdc.cam.ac.uk/ data_request/cif.

[24] ${ }^{3} \boldsymbol{J}_{\mathrm{H} 4, \mathrm{H} 5}=4.7 \mathrm{~Hz}($ calcd $4.1 \mathrm{~Hz}),{ }^{3} \boldsymbol{J}_{\mathrm{H} 5, \mathrm{H} 6}=5.0 \mathrm{~Hz}($ calcd $4.7 \mathrm{~Hz}),{ }^{3} \boldsymbol{J}_{\mathrm{H} 6, \mathrm{H} 7}=$ $6.1 \mathrm{~Hz}$ (calcd $5.9 \mathrm{~Hz}$ ). Coupling constants were calculated with Maestro, Schrçdinger, LLC, New York, NY, 2009. Program PSEUROT as implemented in Matlab GUI (P. M. S. Hendrickx, J. C. Martins, Chem. Cent. J. 2008, 2, 20) was used for the pseudorotational analysis. The ${ }^{3} J_{\mathrm{H} 4, \mathrm{H} 5},{ }^{3} J_{\mathrm{H} 5, \mathrm{H} 6}$, and ${ }^{3} J_{\mathrm{H} 6, \mathrm{H} 7}$ experimental constants were obtained by iterative simulation of the spectra using DAISY as implemented in TOPSPIN v. 2.1 for Bruker. For application of pseudorotational analysis to cyclopentane ring conformation, see: a) C. S. Callam, T. L. Lowary, J. Org. Chem. 2001, 66, 8961 - 8972 b) C. Thibaudeau, A. Kumar, S. Bekiroglu, A. Matsuda, V. E. Marquez, J. Chattopadhyaya, J. Org. Chem. 1998, 63, 5447 - 5462. For a description of the pseudorotation concept, see: c) C. Altona, M. Sundaralingam, J. Am. Chem. Soc. 1972, 94, 8205-8212; d) J. B.
Houseknecht, C. Altona, C. M. Hadad, T. L. Lowary, J.Org. Chem. 2002,67,4647-4651.

[25] Similarly, the vinyl proton of a photoenol obtained by irradiation of 4,4-dimethyl-1-phenyl-1,2-pentanedione is reported to appear at $\mathrm{d}=$ 5.35 ppm; a) P. J. Wagner, R. G. Zepp, K.-C. Liu, M. Thomas, T.-J. Lee, N. J. Turro, J. Am. Chem. Soc. 1976, 98, 8125 - 8134; b) for a recent detection of a long-lived enol from Norrish type II photoreaction of monoketones, see: A. K. Sundaresan, S. Jockusch, N. J. Turro, Photochem. Photobiol. Sci. 2011,10,1450-1454.

26 For this photoenolization of phenyl and alkyl $a$-diketones, see: a) R. G. Zepp, P. J. Wagner, J.Am. Chem. Soc. 1970, 92, 7466- 7467;

b) N. J. Turro, T.-J. Lee, J.Am. Chem. Soc. 1970,92, 7467-7468;

c) R. Bishop, N. K. Hamer, J.Chem. Soc. C 1970,1197-1202;

d) D. S. Weir, J.Chem. Phys. 1962,36,1113-1114.

27 For other examples of pyranose ring inversion induced by bulky protective groups in sugars and $C$-glycosides, see: a) $\mathrm{H}$. Abe, M. Terauchi, A. Matsuda, S. Shuto, J. Org. Chem. 2003, 68, 7439 - 7447; b) T. Hosoya, Y. Ohashi, T. Matsumoto, K. Suzuki, Tetrahedron Lett. 1996, 37, 663 - 666; c) S. Tamura, H. Abe, A. Matsuda, S. Shuto, Angew. Chem. 2003, 115, 1051 - 1053; Angew. Chem. Int. Ed. 2003, 42, 1021 - 1023; d) H. Abe, S. Shuto, A. Matsuda, J.Am. Chem. Soc. 2001, $123,11870-11882$

28 H. Ihmels, J. R. Scheffer, Tetrahedron 1999,55, 885-907.

29 a) R. Bishop, J.Chem. Soc. Chem. Commun. 1972,1288-1289; b) S. Mohr, Tetrahedron Lett. 1980,21, 593-594.

30 Some examples of Yang photocyclization to give hydroxycyclopentanones were originally ascribed to hydrogen abstraction by the innermost carbonyl, but current knowledge makes it more likely to occur directly by the external carbonyl through a seven-membered transition state, see: a) R. Bishop, N. K. Hamer, J. Chem. Soc.C 1970, $1193-$ 1197; b) T. L. Burkoth, E. F. Ullman, Tetrahedron Lett. 1970, 11, 145 148 ; c) see, also reference [1a].

31 The geometry parameters of the 1,5-HAT transition state have been determined for monoketones, and we believe that they can be reasonably extended to the hydrogen abstraction promoted by the internal carbonyl of $a$-diketones. See: a) J. R. Scheffer, C. Scott in Handbook of Organic Photochemistry and Photobiology, Vol. 1 (Eds.: W. Horspool, F. Lenci), CRC, Boca Raton, 2004, p. 54; b) see, also reference [28]

32 For a related uncatalyzed Mukaiyama aldol reaction, see: a) C. T. Wong, M. W. Wong, J. Org. Chem. 2005, 70, 124-131; b) S. E. Denmark, R. A. Stavenger, J. Am. Chem. Soc. 2000, 122, 8837 - 8847; c) K. Miura, H. Sato, K. Tamaki, H. Ito, A. Hosomi, Tetrahedron Lett. 1998, 39, 2585 - 2588; d) S. E. Denmark, B. D. Griedel, D. M. Coe, M. E. Schnute, J.Am. Chem. Soc. 1994,116, 7026-7043;

e) S. E. Denmark, B. D. Griedel, J.Org. Chem. 1994,59,5136-5138; f)S. E. Denmark, B. D. Griedel, D. M. Coe, J. Org. Chem. 1993, 58 , 988 - 990; g) A. G. Myers, S. E. Kephart, H. Chen, J.Am. Chem. Soc.

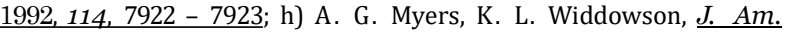
Chem. Soc. 1990,112, 9672- 9674.

33 For the extension of Baldwin's rules to intramolecular aldol condensation, see: a) J. E. Baldwin, L. I. Kruse, J. Chem. Soc. Chem. Commun. 1977, 233 - 234; b) J. E. Baldwin, M. J. Lusch, Tetrahedron 1982,38,2939- 2947.

34 For a review on carbonyl-ene reaction, see: a) M. L. Clarke, M. B. France, Tetrahedron 2008,64,9003-9031; For an early review on ene-reaction, see: b) W. Oppolzer, V. Snieckus, Angew. Chem. 1978, 90.506-516; Angew. Chem. Int. Ed. Engl. 1978, 17, 476-486.

35 For previous examples of ene (oxa-ene) reactions with HAT from an enolic hydrogen, see: a) A. Basheer, Z. Rappoport, J. Org. Chem. 2008, 73, 184 - 190; b) G. Kresze, R. Bussas, Angew. Chem. 1980, 92, 750- 751; Angew. Chem. Int. Ed. Engl. 1980, 19, 737- 739;

c) J. Sander, K. Clauss, Angew. Chem. 1980, 92, 138 - 139; Angew. Chem. Int. Ed. Engl. 1980, 19, 131 - 131; d) K. Kociolek, M. T. Leplawy, Synthesis 1977, 778 - 780; e) R. Bloch, P. Le Perchec, F. Roues- sac, J.-M. Conia, Tetrahedron 1968,24,5971-5989.

36 For a related example of uncatalyzed carbonyl-ene reaction, see: $\mathrm{H}$. Helmboldt, M. Hiersemann, J.Org. Chem. 2009,74, 1698-1708, 
37 For an example of steric acceleration in an ene reaction, see: a) N. Choony, P. G. Sammes, G. Smith, R. W. Ward, Chem. Commun. 2001, 2062 - 2063; b) N. Choony, N. Kuhnert, P. G. Sammes, G. Smith, R. W. Ward, J.Chem. Soc. Perkin Trans. 1 2002,1999- 2005.

38 a) T. Bekele, C. F. Christian, M. A. Lipton, D. A. Singleton, J. Am. Chem. Soc. 2005, 127, 9216-9223; b) D. A. Singleton, C. Hang, $J$. Am. Chem. Soc. 1999, 121, 11885-11893; c) D. A. Singleton, C. Hang, Tetrahedron Lett. 1999, 40, $8939-8943$; d) Z. Song, P. Beak, $\underline{J}$. Am. Chem. Soc. 1990, 112, 8126-8134; e) B. B. Snider, E. Ron, J. Am. Chem. Soc. 1985, 107, 8160-8164; f) C.-C. Cheng, C. A. Seymour, M. A. Petti, F. D. Greene, J. Org. Chem. 1984, 49, 2910- 2916; g) H. Kwart, M. W. Brechbiel, J.Org. Chem. 1982, 47, 3353-3355; h) O. Achmatowicz, J. Szymoniak, J.Org. Chem. 1980, 45, 47744776; i) S.-H. Dai, W. R. Dolbier, J.Am. Chem. Soc. 1972, 94,3953$\underline{3954}$.

39 a) D. F. Cauble, J. D. Gipson, M. J. Krische, J.Am. Chem. Soc. 2003 $\underline{125,1110-1111 ; b) ~ H . ~ E . ~ Z i m m e r m a n, ~ M . ~ D . ~ T r a x l e r, ~ J . A m . ~ C h e m . ~}$ Soc. 1957,79, 1920-1923.

40 5-Hydroxy-2,3-pentanedione (laurencione), a naturally occurring $a$ diketone isolated from the red alga Laurencia spectabilis, also exists preferentially in a cyclic hemiketal form (2-hydroxy-2-methyldihydro-3(2H)-furanone); a) M. W. Bernart, W. H. Gerwick, E. E. Corcoran, A. Y. Lee, J. Clardy, Phytochemistry 1992,31, 1273-1276; For a synthesis, see: b) N. De Kimpe, A. Georgieva, M. Boeykens, L. Lazar, J.Org. Chem. 1995, 60,5262-5265.
41 For ring opening of related a-hydroxycyclobutanones with periodic acid, see: W. H. Wry, J. C. Duggan, M.-s. H. Pai, J. Am. Chem. Soc. 1970, 92, 5785- 5787.

42 a) P. de Armas, C. G. Francisco, E. Suárez, Angew. Chem. 1992, 104, 746 - 748; Angew. Chem. Int. Ed. Engl. 1992, 31, 772 - 774; b) C. C. González, A. R. Kennedy, E. I. León, C. Riesco-Fagundo, E. Suárez, Angew. Chem. 2001,113, 2388- 2390; Angew. Chem. Int.Ed. 2001, 40, 2326-2328.

43 J. L. Chiara, S. Bobo, E. Sesmilo, Synthesis 2008,3160-3166.

44 a) M. Miljkovic, M. Habash-Marino, J.Org. Chem. 1983, 48, 855 - 860; b) R. P. Spencer, C. L. Cavallaro, J. Schwartz, J.Org. Chem. 1999, 64,3987- 3995.

45 a) N. Merbouh, J. M. Bobbitt, C. Brueckner, TetrahedronLett. 2001, 42, 8793 - 8796; b) A. Borbás, G. Szabovik, Z. Antal, K. Fehér, M. Csávás, L. Szilágyi, P. Herczegh, A. Lipták, Tetrahedron: Asymmetry 2000,11, 549 - 566; c) R. Bihovsky, C. Selick, I. Giusti, J. Org. Chem. 1988,53,4026-4031.

46 H. D. K. Drew, E. H. Goodyear, W. N. Haworth, J.Chem. Soc. 1927, 1237-1245.

47 a) S. Sasaki, Y. Taniguchi, R. Takahashi, Y. Senko, K. Kodama, F. Nagatsugi, M. Maeda, J.Am. Chem. Soc. 2004,126, 516-528; b) J. A. Piccirilli, T. Krauch, L. J. MacPherson, S. A. Benner, $\underline{\text { Helv}}$. Chim. Acta 1991,74, 397-406.

Received: April 1, 2013 Published online: June 20, 2013 